\title{
Burkina Faso: Fourth Review Under the Three-Year Arrangement Under the Extended Credit Facility and Request for Modification of Performance Criteria and Augmentation of Access-Staff Report; Debt Sustainability Analysis; Press Release on the Executive Board Discussion; and Statement by the Executive Director for Burkina Faso.
}

In the context of the fourth review under the three-year arrangement under the extended credit facility and request for modification of performance criteria and augmentation of access, the following documents have been released and are included in this package:

- $\quad$ The staff report for the Fourth Review Under the Three-Year Arrangement Under the Extended Credit Facility and Request for Modification of Performance Criteria and Augmentation of Access, prepared by a staff team of the IMF, following discussions that ended on April 4, 2012, with the officials of Burkina Faso on economic developments and policies. Based on information available at the time of these discussions, the staff report was completed on May 24, 2012. The views expressed in the staff report are those of the staff team and do not necessarily reflect the views of the Executive Board of the IMF.

- $\quad$ A Joint IMF/World Bank Debt Sustainability Analysis.

- $\quad$ A Press Release summarizing the views of the Executive Board as expressed during its June 8, 2012 discussion of the staff report that completed the request and/or review.

- $\quad$ A statement by the Executive Director for Burkina Faso.

The documents listed below have been or will be separately released.

Letter of Intent sent to the IMF by the authorities of Burkina Faso* Memorandum of Economic and Financial Policies by the authorities of Burkina Faso*

*Also included in Staff Report

The policy of publication of staff reports and other documents allows for the deletion of market-sensitive information.

\author{
Copies of this report are available to the public from \\ International Monetary Fund • Publication Services \\ $70019^{\text {th }}$ Street, N.W. • Washington, D.C. 20431 \\ Telephone: (202) 623-7430 • Telefax: (202) 623-7201 \\ E-mail: publications@imf.org Internet: http://www.imf.org
}

\section{International Monetary Fund Washington, D.C.}




\title{
INTERNATIONAL MONETARY FUND
}

\section{BURKINA FASO}

\section{Fourth Review Under the Three-Year Arrangement Under the Extended Credit Facility and Request for Modification of Performance Criteria and Augmentation of Access}

\author{
Prepared by the African Department \\ (In consultation with other departments) \\ Approved by Michael Atingi-Ego and Thomas Dorsey
}

May 24, 2012

\begin{abstract}
Discussions and outreach: Held in Ouagadougou during March 21-April 4. The mission met with Economy and Finance Minister Bembamba, Deputy Minister in charge of Budget Zoundi, BCEAO National Director Ki-Zerbo, other senior officials, private sector representatives, development partners, and the local press. The mission did presentations on the global outlook, selected country responses to higher international fuel prices, and the FARI model for mining revenues. The mission held a press conference and issued a press statement at its conclusion.
\end{abstract}

IMF team: Ms. Redifer (head), Ms. Adenauer (resident representative), Mr. van Houtte, Ms. Diouf, and Mr. Wiseman (all AFR), and Mr. Ouattara (local IMF office). Mr. Tall (OED) joined in policy discussions, and Mr. Atingi-Ego (AFR) joined policy discussions in the final days of the mission.

Fund relations: The three-year ECF-supported program was approved by the Executive Board on June 14, 2010 in an amount of SDR 46.15 million (77 percent of quota). The Executive Board approved the first three reviews of the program and discussions for the 2011 Article IV consultation took place in December 2011.

Fourth review of the ECF-supported program: All end-December quantitative performance criteria were respected. Most structural benchmarks were met, although some with a delay. The authorities have implemented significant fiscal adjustment and sought new donor commitments to meet new needs arising from a food security crisis and an influx of Malian refugees, but this will not be enough to meet the large external and fiscal needs. To catalyze additional donor financing and safeguard implementation of their development program, the authorities are therefore requesting an augmentation of program access in the amount of 60 percent of quota. The augmentation would be disbursed in two tranches, with a larger tranche disbursed with this review to meet immediate external financing needs and catalyze additional external support. Staff supports the authorities' request and proposes modified targets and structural benchmarks for end-June 2012. 


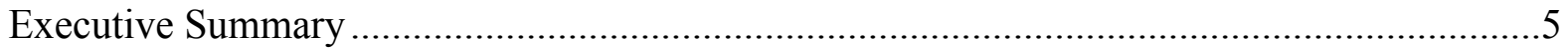

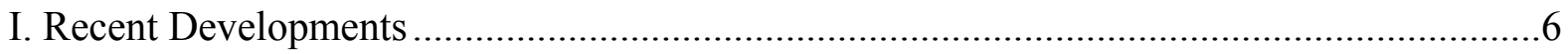

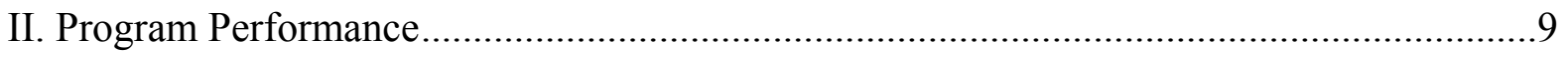

III. Outlook and Policy Discussions ............................................................................. 10

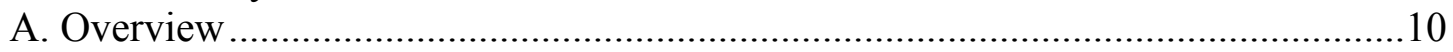

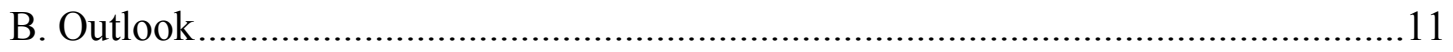

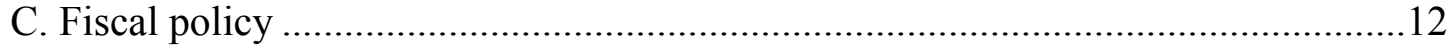

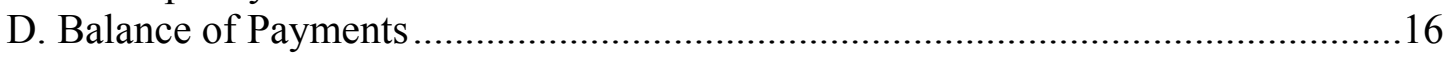

E. Financial Sector Policies .............................................................................. 16

F. Debt Sustainability and External Borrowing ................................................... 17

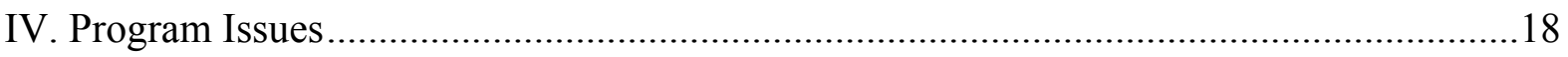

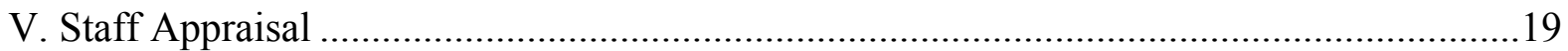

Tables

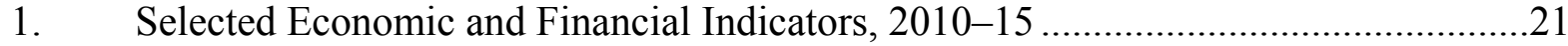

2a. Consolidated Operations of the Central Government, 2010-15 ..............................22

2b. Consolidated Operations of the Central Government, 2010-15 ................................23

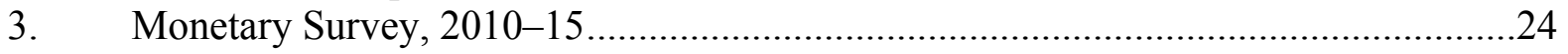

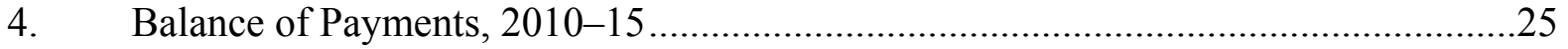

5. Financing Requirements, 2011-14 …..............................................................26

6. Quantitative Assessment Criteria and Indicative Targets, 2011 .............................27

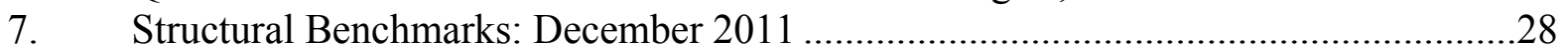

8. Poverty-Reducing Social Expenditure, 2005-12 ..............................................29

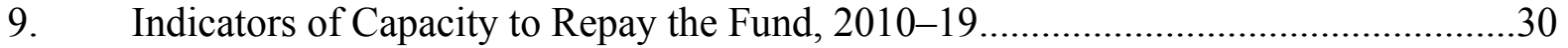

10. Schedule of Disbursements Under the ECF Arrangement, 2010-13 .......................31

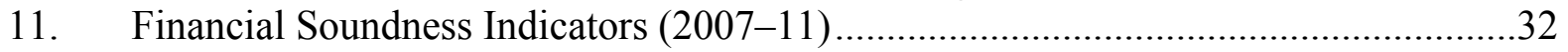

\section{Boxes}

1. Coping with Food Insecurity and Malian Refugees ............................................... 9

2. The Strategy for Accelerated Growth and Sustained Development (SCADD) ...........10

3. Eliminating Fuel Price Subsidies ................................................................... 12

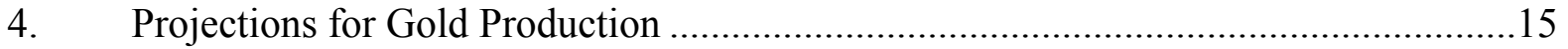

Figures

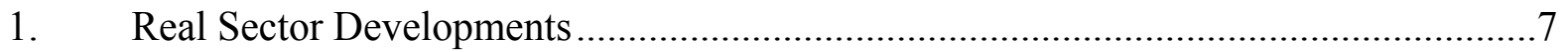

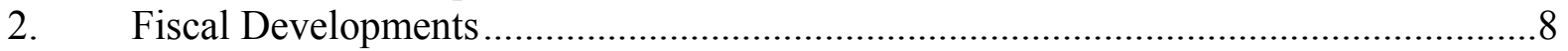




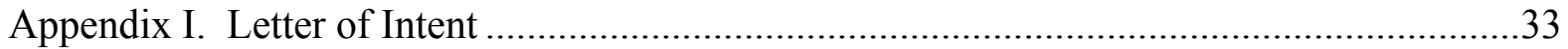
Attachment I. Memorandum on Economic and Financial Policies for 2012-13 ........35 Attachment II. Technical Memorandum of Understanding .....................................50 


\section{List of Acronyms}

BCEAO Central Bank of West African States

CPIA

Country Policy and Institutional Assessment

DeMPA

Debt Management Performance Assessment

MEFP

Memorandum of Economic and Financial Policies

MDGs

Millenium Development Goals

MTDS

NPV

Medium-Term Debt Strategy

PFM

Net Present Value

QPC

Public Financial Management

SCADD

Quantitative Performance Criterion

Stratégie pour une Croissance Accélérée et pour le Développement Durable (Strategy for Accelerated Growth and Sustainable Development)

SSA sub-Saharan Africa

SOFITEX Société Burkinabè des Fibres Textiles (the largest cotton ginning company)

SONABHY

Société Nationale des Hydrocarbures (public oil importing company)

WAEMU

West African Economic and Monetary Union 


\section{Executive Summary}

Burkina Faso faces a very challenging policy environment, with multiple exogenous shocks. Drought in 2011 created a food shortage that has placed about 10 percent of the population in a situation of food insecurity. An influx of refugees from Mali (56,700 as of mid-May) is exacerbating the shortages. The authorities devised a well-targeted and phased program to handle domestic food shortages, but only about half of the needed financing has been secured. The refugees are currently receiving international humanitarian aid, but aid provision is inadequate and living conditions are growing increasingly dire.

Near term policy discussions focused on specifying 2012 financing needs arising from the shocks to help prevent crowding out the authorities' development program, the SCADD. After taking into account spending needs, domestic adjustment measures and new donor financing, estimated remaining financing needs are roughly CFAF 55.7 billion (1 percent of GDP), but could be higher if the refugee situation worsens. If the resources cannot be mobilized or come very late in the year, spending for the development program would likely need to be reallocated to meet crisis needs.

The authorities have implemented decisive adjustment measures, without which financing needs would be much higher. Most notable was the authorities' decision to increase retail fuel prices, halving an implicit subsidy, and set in place an automatic price adjustment mechanism to gradually eliminate the remaining subsidy and enable full passthrough of international prices. Tax administration measures and higher mining royalties have enhanced revenues, combined with measures to contain public wages.

Program performance was strong in 2011, despite domestic social unrest and political turmoil in Côte d'Ivoire, the main coastal access. All quantitative performance criteria were met, as were almost all indicative targets. Most structural reforms were met, albeit with some delay. Revenue reached 16.5 percent of GDP, compared to 15.1 percent anticipated, and the fiscal deficit was reduced from 4.5 percent of GDP in 2010 to 2.5 percent.

Over the medium term, the SCADD provides a good platform for sound macroeconomic policy, improving resilience to shocks, and accelerating progress toward the MDGs. The authorities' priority for the medium term is implementation of SCADD and creation fiscal space to accommodate it. To this end, they have specified medium-term measures to boost revenues further and contain non-priority current spending. The SCADD includes immediate measures to diversify agricultural production, expand irrigation, and improve food distribution, while establishing a targeted social safety net.

Staff supports the authorities' request to complete the fourth review and augment program access by 60 percent of quota. Balance of payment needs reflect higher food imports to respond to the crises. A new joint DSA with the World Bank indicates much lower risk of debt distress, suggesting that additional IMF borrowing would put a negligible strain on debt sustainability. 


\section{RECENT DEVELOPMENTS}

1. Real growth in 2011 was lower than expected (Figure 1). Real GDP growth is estimated to have been 4.2 percent, as a result of a sharp contraction in cereal production owing to poor rainfall, and the impact of a political crisis in Côte d'Ivoire. Domestic social unrest earlier in 2011 affected growth only modestly, because it largely affected the informal sector, and the government provided rapid loans to keep small businesses from closing. Growth was supported by gold and cotton production and the services sector, and growth in credit to the private sector remained robust.

2. Driven by food prices, inflation increased in late 2011 (Figure 2). Year-over-year inflation reached 5.1 percent in December 2011, and average annual inflation was up to 2.7 percent from negative 0.6 percent in 2010. Inflation decelerated sharply in early 2012 because of government sales of food at below market prices in January, with the CPI registering a year-over-year increase of 2.8 percent.

3. The fiscal deficit was reduced sharply in 2011. Revenue collection reached 16.5 percent of GDP, exceeding expectations, due to improvements in revenue administration, higher mining royalties and profit taxes, and a one-off windfall in the collection of estimated corporate income taxes. ${ }^{1}$ Additional unanticipated spending to contain the impact of political unrest in early 2011 (of roughly 0.8 percent of GDP) did not outpace lower execution of the programmed budget in the first half of the year. As a result, the fiscal deficit was almost halved, from 4.5 percent of GDP in 2010 to 2.5 percent.

4. The current account deficit narrowed in 2011. Strong gold production, combined with a sharp increase in international gold and cotton prices, turned the trade balance positive, despite higher fuel and food prices. In spite of poor rainfall for much of the growing season, cotton production remained robust, helped by new irrigation practices.

\section{The 2012 Sahel drought caused serious food shortages, which have been} exacerbated by an influx of refugees from Mali (Box 1). A contraction of food production in the region, together with ongoing structural food deficits, is expected to have placed about 10 percent of the population into a situation of food insecurity. On top of this, starting in early 2012, conflict in the north of Mali caused the local population to take refuge in Burkina Faso. As of mid-May, there were 56,700 refugees (and cattle), living in increasingly dire humanitarian conditions. Increasing fuel prices have added further stress.

\section{The euro zone developments, however, pose little threat beyond potential}

reductions in aid. Burkina Faso's financial sector is supported by local deposits, exports are relatively inelastic to growth in Europe, and remittances are small.

\footnotetext{
${ }^{1}$ Taking out a one-time payment of estimated corporate taxes, revenues would have been 15.9 percent of GDP vs. 15.1 percent expected at the time of the third review.
} 
Figure 1. Burkina Faso: Real Sector Developments

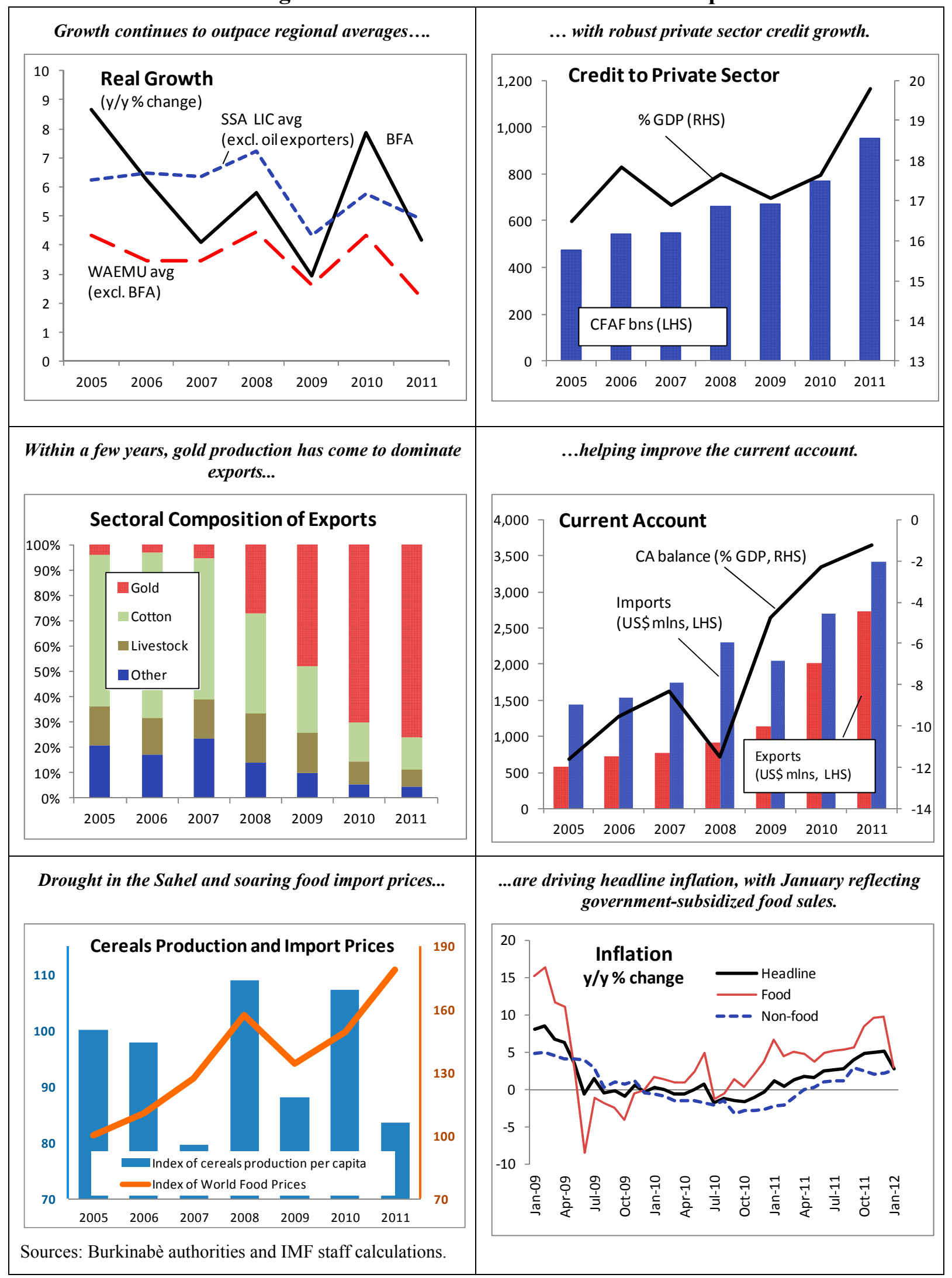


Figure 2. Burkina Faso: Fiscal Developments

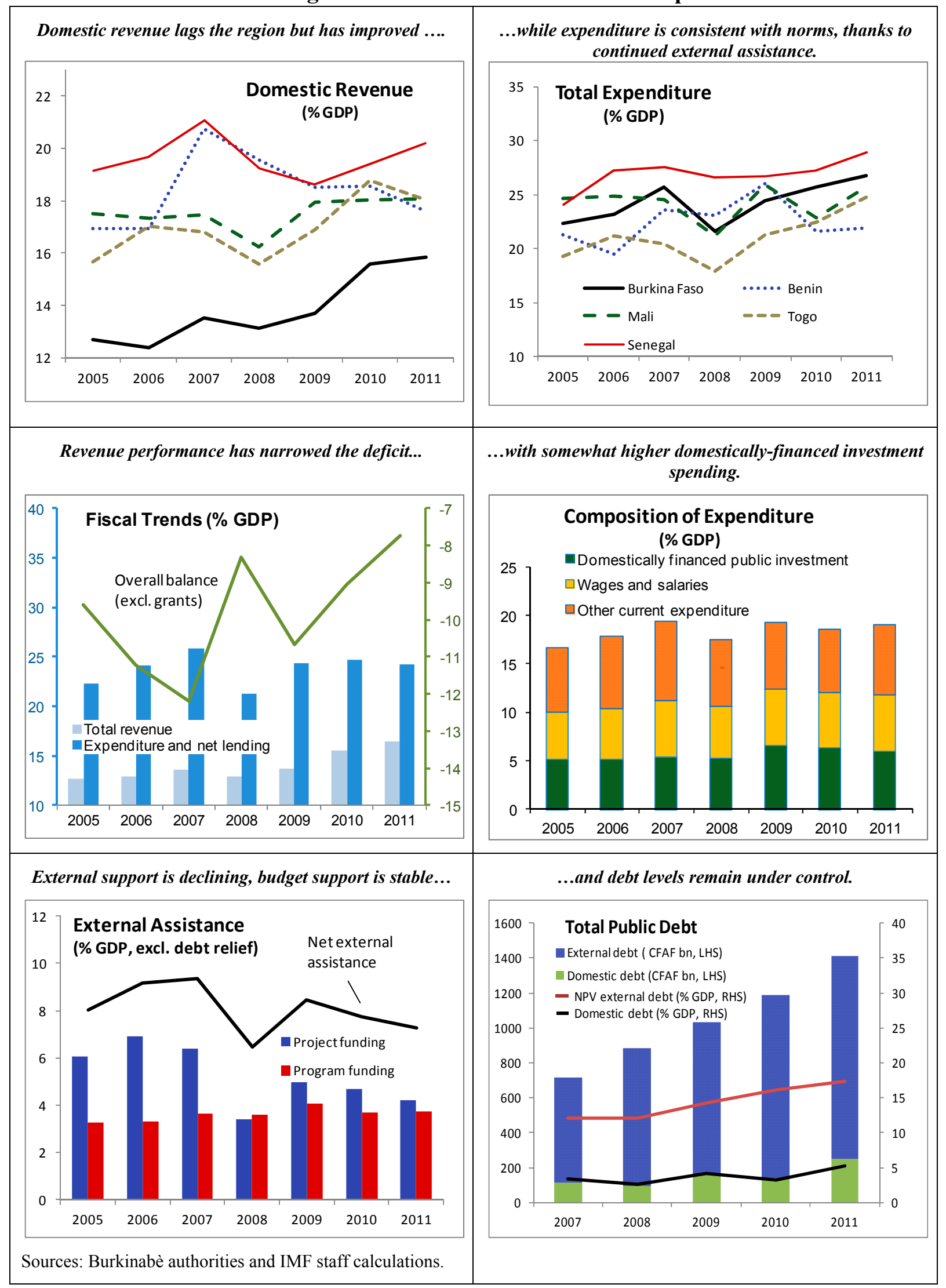




\section{Box 1. Coping with Food Insecurity and Malian Refugees}

The Sahel drought took a heavy toll on grain production in Burkina Faso. Inadequate rain reduced the 2011 harvest, especially grain production which dropped by 19.6 percent compared to 2010. With a cereal deficit estimated at about 155,000 tonnes, 30 percent of consumption needs will remain uncovered leaving more than one million Burkinabè without access to adequate food.

The Burkinabè authorities designed a four-phase program to address food security. The strategy has been tailored to address evolving needs for each quarter of 2012 while minimizing waste. Households are categorized under four levels of need that intensify over time as resources are depleted. The mix of measures will evolve as households' food needs become more urgent. Measures include:

$\checkmark$ Public local shops providing food at below market prices;

$\checkmark$ In-kind or cash payments in exchange for work;

$\checkmark$ Food distribution and cash transfers to the most vulnerable;

$\checkmark$ Early warning systems to monitor food shortages in the provinces; and

$\checkmark$ Distribution of high yield seeds and subsidized inputs for the 2012/13 grain harvest.

Implementation of the strategy is already underway and is expected to cost about CFAF 112 billion (US\$224 million). In addition to 6,000 tonnes of grain delivered in Q1, about 50,000 tonnes will be made available for subsidized sale/distribution in April-June, and about 100,000 tonnes for the rest of the year. The needs are partly structural. Donors have pledged about CFAF 43.3 billion. The 2012 original budget authorized CFAF 18.8 billion.

The influx of Malian refugees is adding strain to food security problems. Recent turmoil in northern Mali has induced a flow of refugees to neighboring countries, including Burkina Faso. The number of refugees was expected to reach 60,000 by end June and on this basis, in consultation with UNHCR, the authorities estimated financing needs of about CFAF 13 billion. Donor financial pledges as of April were CFAF 2.3 billion. As of May 10, the number of refugees was already 56,700.

\section{Program Performance}

7. Quantitative program targets have been respected, despite the shocks (Table 6). For end-September and the end-December test dates, data indicate that the quantitative criterion on the overall deficit (commitment basis) was met with a comfortable margin, all borrowing ceilings were respected, and the indicative target on revenue collection was exceeded by a large margin. Despite spending to dampen unrest, which caused delays in social spending, the indicative floor on social spending was missed by only a small amount (94 percent was executed), which also reflects a short delay into early 2012 of a World Bank education disbursement.

\section{Implementation of structural reforms was mixed, but the most important} measure was implemented (Table 7). The authorities implemented the politically most difficult structural benchmark: adopting a new pricing and taxation system for petroleum products. The measure was adopted in early April 2012 following consultations with trade associations and other stakeholders to help prevent a repeat of the social unrest from early 2011. Although a one-stop window for customs clearance and an electronic system to 
improve merchandise valuation in two customs posts were set up, these are not yet operational. Follow-on structural benchmarks have been added for end-2012 to ensure that these systems are working as intended by early 2013 (MEFP $\$ 36$ ). The authorities have conducted ongoing surveys to check against the payroll records and civil service roster, and they plan to expand on this measure by introducing biometric cards for public servants (MEFP q37). Although the Cabinet adopted a new strategy to develop the microfinance sector, it has not yet been put in place. Therefore, a new structural benchmark to monitor progress toward the implementation of the strategy has been added for June 2012 (MEFP \ 40). Expenditure verification units were established in five key ministries to help reduce execution delays, meeting a structural benchmark for end-March 2012. The units will become fully operational in the second half of 2012 (MEFP ๆ34).

\section{Outlook ANd Policy Discussions}

\section{A. Overview}

\section{Policy discussions centered on how to respond to the current crises, while} preserving fiscal space to implement the authorities' development program. The financing needs created by the current food security crisis and the influx of Malian refugees pose a significant threat to crowding out expenditure of the authorities' development plan, the SCADD (Box 2), which is designed, among other investment objectives, to help improve the country's resilience to exogenous shocks.

\section{Box 2. The Strategy for Accelerated Growth and Sustained Development (SCADD)}

Building on progress during 2000-10, the SCADD reorients development policies with the objective of accelerating progress toward the MDGs. The SCADD has four strategic axes: (i) generating sustained and inclusive growth; (ii) developing human capital and promoting social protection; (iii) strengthening governance; and (iv) promoting cross-cutting policies, e.g., gender and environment.

For the growth axis, agricultural development is aimed at eliminating structural food deficits and creating more resilience to climatic shocks. Irrigation techniques already in use will be expanded, as will access to higher productivity inputs, such as enhanced seeds and fertilizer, and inputs for new cash crops, such as sesame and beans. Further agricultural research will support dissemination of more productive farming and post-harvest techniques. Strategic development of the road network should improve food distribution and access to markets, supported by price transparency schemes and early warning systems for food shortages.

Beyond agriculture, the SCADD plans other diversification measures, for example, incentives to expand manganese and zinc mining. Public investment in infrastructure is planned, particularly to secure more reliable energy supplies, cheaper transportation, and reduction of other factor costs. Several measures to improve the business environment and access to financial services are also included.

The government's social protection policy includes as key elements: (i) cash transfers and food vouchers for the poorest households; (ii) expanded provision of medical services and free primary education, school lunches, and prenatal care in poorer regions; (iii) employment opportunities, including food-for-work schemes; and (iv) social insurance mechanisms.

The authorities have drawn up a detailed action plan with time lines and costing, incorporated into the medium term expenditure framework and yearly budgets. Donors have pledged multi-year commitments to support the SCADD, but there is risk that these resources will be diverted in light of current crisis spending needs. 
10. To help meet new financing needs, the authorities have implemented decisive adjustment measures. In the absence of these measures, staff estimates that the residual financing needs for 2012 would be at least twice as high:

- Better-than-expected improvements in revenue performance are assumed to be maintained in 2012, with the 2012 revenue projection boosted from 15.5 percent of GDP at the third review to 16.1 percent currently.

- To stem losses by the state-owned oil company, the government increased retail fuel prices, eliminating half of the current implicit subsidy. It set in place an automatic price adjustment mechanism to gradually eliminate the remaining subsidy and provide for full pass-through of international prices thereafter (Box 3).

- Civil service allowances and per diems were reduced in 2011. Additional measures are underway (MEFP, $\mathbb{\top} 11)$ to reduce fraud and redundant benefits to contain the wage bill.

\section{B. Outlook}

11. The authorities and the mission agreed that growth projections in $\mathbf{2 0 1 2}$ and over the medium term should be increased to 7.0 percent. For 2012, this largely reflects an anticipated recovery in the cereal harvest in the fall. This recovery, and agriculture more generally, will be supported by measures under the SCADD to expand irrigation, diversification, and the use of enhanced seeds for greater output. Beyond 2012, the growth prospects reflect intensification of SCADD measures, including the establishment of growth "poles," but also concrete plans to expand gold and other mining production over the next 3 to 4 years. Inflation projections for 2012 were increased to 3.0 percent for the year, based on volatile food prices and the direct and indirect impacts of higher retail fuel prices.

\section{The external current account deficit is expected to worsen significantly in} 2012 and improve gradually over the medium term. Large imports in 2012 reflect the needs of the food security program and the Malian refugees, as well as higher international fuel prices. The authorities' infrastructure investment program will also increasingly put pressure on the current account over the medium term. However, gold exports are expected to continue to grow significantly over the medium term, triggering an overall improvement in the trade balance. Cotton exports are also expected to grow through 2013, reflecting the authorities' production and price projections, with conservative assumptions thereafter. ${ }^{2}$

\footnotetext{
${ }^{2}$ The WEO assumes a sharp drop in cotton prices in 2012, but the authorities took issue with this assumption based on forward-contracts and the higher-quality niche market of Burkina cotton.
} 


\section{Box 3. Eliminating Fuel Price Subsidies}

As in many countries, Burkina Faso's fixed retail fuel prices gave rise to growing contingent liabilities in the face of sharp increases in international fuel prices. The stateowned oil company, SONABHY, began experiencing operational losses in 2011, which accelerated rapidly in early 2012. Its credit lines with commercial banks were under threat, so in late March 2012 the government took accepted the accrued losses as an explicit liability. The government made a CFAF 15.5 billion cash transfer and issued a 10 year bond for CFAF 38.3 billion to SONABHY (with interest costs of CFAF 0.6 billion in 2012), which will undergo restructuring to reduce operating costs.

To phase out the subsidy going forward, the government undertook two measures. First, on March 31, after several consultations with trade associations and other stakeholders to inform them about the unsustainable situation, it implemented an increase of CFAF 50 on all fuel categories, equivalent to about half of the implicit subsidy and around 7.5 percent of retail prices for gasoline and diesel. Second, on April 4, it adopted a new Oil Price Adjustment Mechanism (OPAM) to gradually eliminate the remainder of the subsidies. The OPAM would allow the gap between international and local retail prices to close in as few as two months. Had the government not taken these measures, SONABHY losses for 2012 could easily have been twice as high as 2011 , since monthly losses doubled in the first three months of 2012.

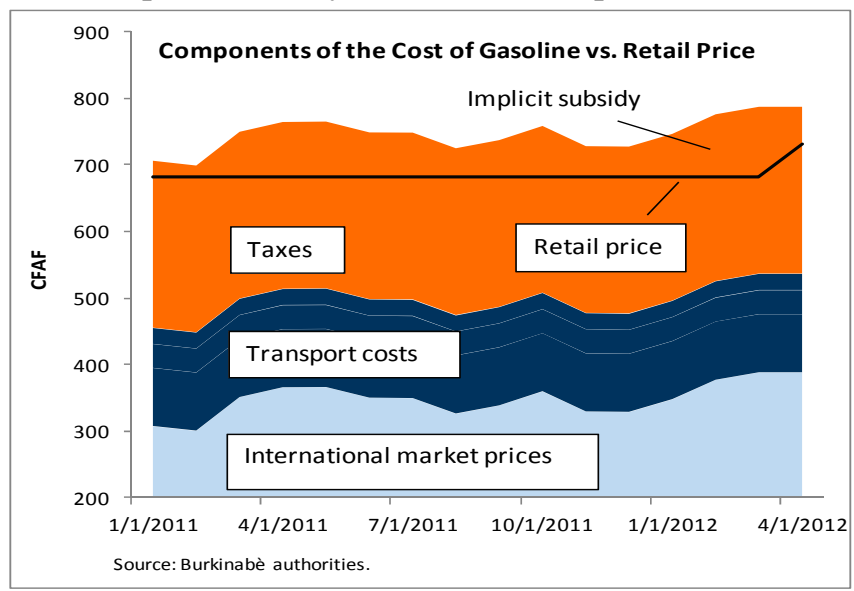

There is limited discretion under OPAM to avoid passing through international price increases. A commission will undertake price reviews once per month, and make a recommendation to the Ministers of Finance and Commerce for their joint endorsement. The price adjustment corresponds to the average of the international price for the first 25 days of the past month, plus a mark-up to reflect import and distribution costs and taxes. Ministers choose the date on which these measures come into force (within a month): monthly and yearly increases are limited to CFAF 50 and 100 per liter, respectively.

\section{Fiscal Policy}

13. External financing needs for the food security crisis and the Malian refugees for the 2012 are estimated at CFAF 61 billion (US\$122 million), with the bulk of the need in the third quarter (Text Table 1). Donors have pledged roughly CFAF 46.6 billion (US\$94 million) in new financing to respond to shocks this year. However, both the size and timing of donor support is insufficient. Financing needs are concentrated in the third quarter (CFAF 39.2 billion) when household reserves will have been depleted and before the fall harvest. 
Text Table 1. Burkina Faso: Estimation of External Financing needs for 2012 (CFAF billions)

\begin{tabular}{|c|c|c|c|c|c|}
\hline & Q1 & Q2 & Q3 & Q4 & 2012 \\
\hline Total new spending needs & 20.9 & 34.6 & 55.4 & 14.6 & 125.5 \\
\hline Food security program ${ }^{1}$ & 20.9 & 34.6 & 48.9 & 8.1 & 112.5 \\
\hline Malian refugees $(60,000)^{2}$ & 0.0 & 0.0 & 6.5 & 6.5 & 13.0 \\
\hline Total resources & 20.9 & 14.0 & 16.8 & 12.7 & 64.5 \\
\hline Budgeted resources & 14.0 & 4.8 & 0.0 & 0.0 & 18.8 \\
\hline Of which: food security & 14.0 & 4.8 & 0.0 & 0.0 & 18.8 \\
\hline refugees & 0.0 & 0.0 & 0.0 & 0.0 & 0.0 \\
\hline Donor pledges for food security & 6.9 & 9.2 & 15.6 & 11.5 & 43.3 \\
\hline Donor pledges for refugees & 0.0 & 0.0 & 1.2 & 1.2 & 2.3 \\
\hline of which: program grants & 0.0 & 0.0 & 0.0 & 0.0 & 0.0 \\
\hline project grants & 0.0 & 0.0 & 1.2 & 1.2 & 2.3 \\
\hline Difference & 0.0 & 20.5 & 38.6 & 1.9 & 61.0 \\
\hline Previous gap ${ }^{3}$ & 0.0 & 4.8 & 0.0 & 4.8 & 9.6 \\
\hline Total remaining needs & 0.0 & 25.3 & 38.6 & 6.7 & 70.6 \\
\hline \multicolumn{6}{|c|}{ Sources: Burkinabè authorities; and IMF staff estimates and projections. } \\
\hline \multicolumn{6}{|c|}{$\begin{array}{l}{ }^{1} \text { More than half of supplies to be imported, and all the residual needs are imports, since } \\
\text { the authorities redistributed domestic production and reserves first. }\end{array}$} \\
\hline \multicolumn{6}{|c|}{${ }^{2}$ Cost is in addition to in-kind humanitarian assistance. } \\
\hline${ }^{3}$ Prospective IMF disbursement & CF pro & review. & & & \\
\hline
\end{tabular}

\section{Other changes to spending and resource projections result in a surplus of} CFAF 14.9 billion, which can be used to offset part of the external financing needs for the year (Text Table 2). New spending needs were identified, including unbudgeted costs of parliamentary and local elections, the workout for the state-owned oil company, subsidies to support cotton inputs, and revised execution assumptions about externally financed investment spending based on historical trends. In addition, new projections were made for the domestic resource envelope, based on gains in revenue administration and increased mining royalties, as well as planned and already-executed bond issuances for 2012. External project financing was adjusted in line with the spending side.

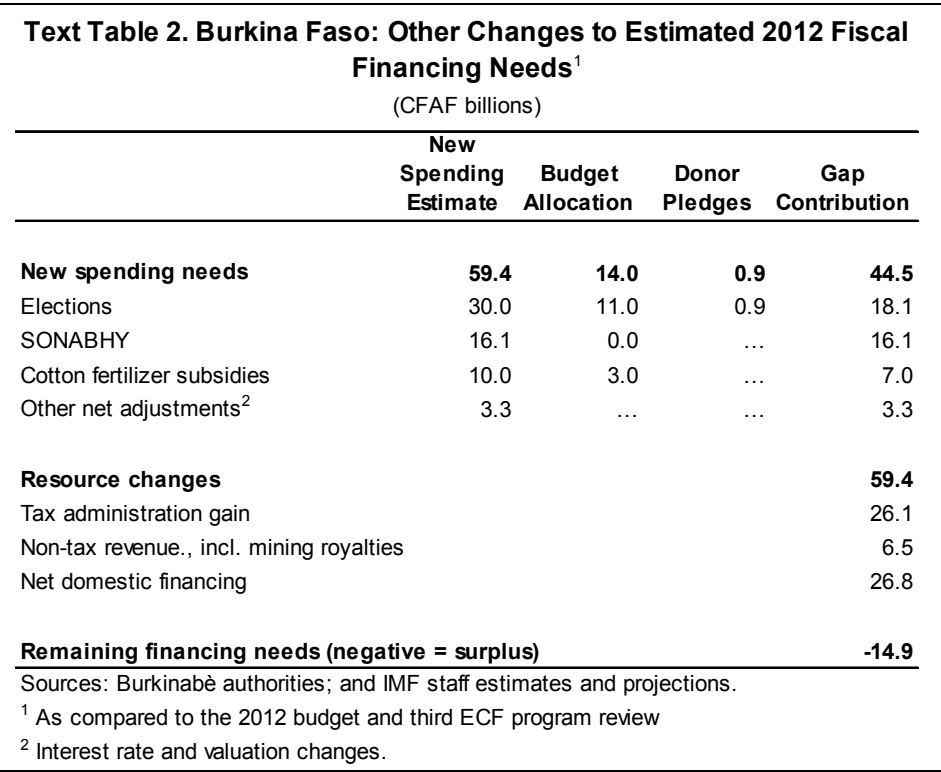


15. This leaves remaining external financing needs for the year of CFAF 55.7 billion (US\$112 million or 1.0 percent of GDP). However, this estimate is based on a very conservative assumption that the number of refugees from Mali does not exceed the projection for end-June of 60,000 people, whereas the UNHCR has estimated that the number could triple by the end of the year. The authorities are working closely with other donors to explore other sources for financing and to ensure existing financing is disbursed on time. They have met 10 prior actions to ensure that an expected World Bank structural grant of US\$70 million is disbursed in July, and have requested that the Bank increase the grant in light of the shocks. IMF staff is also meeting with donors to explore their financing intentions.

16. To help meet needs for additional food imports, the authorities have requested an augmentation of access under the ECF-supported program in the amount of 60 percent of quota (US\$55.38 million). They have requested that the disbursement be frontloaded as much as possible in order to meet the peaking needs of the food security program over the summer. This, plus disbursements already planned, would leave remaining financing needs of US\$36.6 million (CFAF 18.3 billion), which are reflected in the macroeconomic framework as unidentified program grants. Should additional financing to meet the remaining needs not materialize, the authorities will meet the spending gap through a combination of additional domestic financing allowed for under the program and spending adjustment, likely in the area of domestically-financed investment, putting implementation of the SCADD at risk.

17. Beyond 2012, fiscal policy is aimed at creating fiscal space for implementation of the SCADD, through continued improvements in revenue collection and containment of non-priority spending. The authorities are undertaking a number of measures to maintain the momentum for revenue collection, including improving collection of tax arrears, spreading tax controls, setting up a one-stop window for customs clearance to streamline processing and reduce fraud, auditing customs clearance procedures in Ouagadougou, rolling out in three additional tax units a new electronic system to manage revenue collection, and improving information sharing between the tax and customs departments via the interconnection of their electronic systems (MEFP, ๆ 11, 21, and 35-36). Plans to expand gold production and diversify into other areas of mining should also help support revenues, and the authorities are currently reviewing their mining code/taxation regime to ensure that it is in line with international norms (Box 4). To help contain spending, the authorities hope to limit current transfers through measures to strengthen the state-owned oil and electricity companies, and 


\section{Box 4. Projections for Gold Production}

The production of gold in Burkina Faso has grown dramatically over the past four years. A new mining code in 2003 and investment incentives spurred a rash of exploration and development for gold, as well as manganese and zinc. What was once a small-scale, semi-traditional industry has transformed into a driver of growth and the dominant export.

Burkina Faso currently has eight mines in operation that will continue their current output past 2020. With more than 50 projects currently in exploration or development of new sites, gold production growth could peak at several times its current value. Based on the import of two new crushers this year and other expansion plans, export projections have been updated to reflect an increase of 14 tonnes in the next three years, peaking in 5-8 years at around 70 tonnes. These projections are cautious relative to past years' exponential growth and the number of projects under development.

The mission team made a wellreceived presentation to the

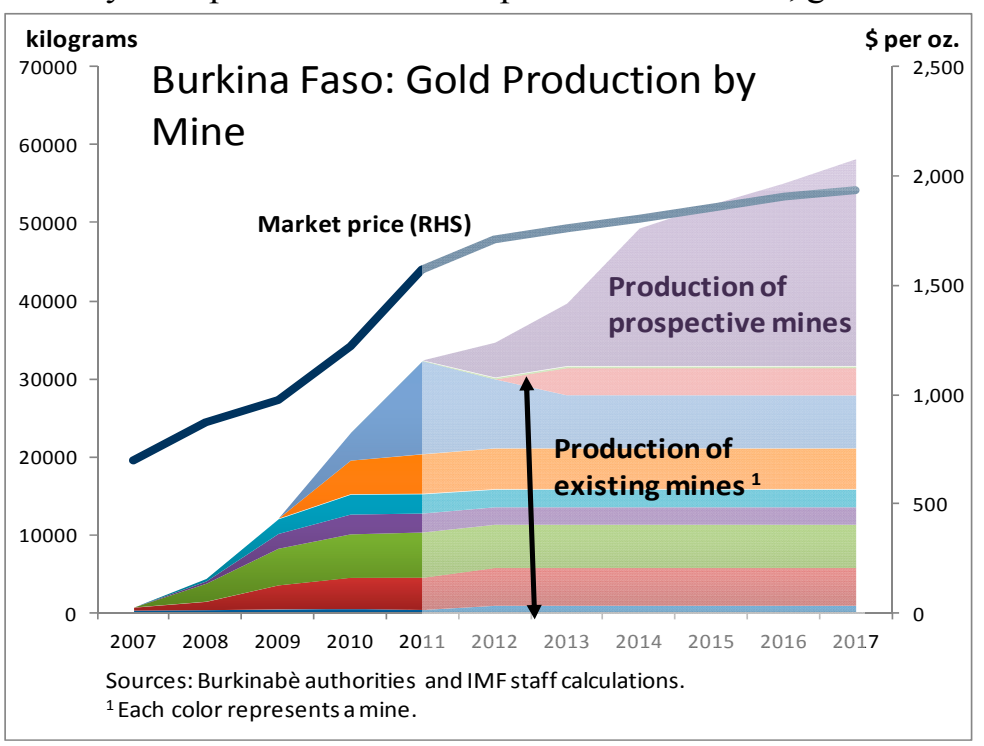
authorities on FAD's FARi model for estimating resource production and revenue projections, as well as comparing mining taxation regimes to comparator countries. Several officials commented that improving local technical capacity to examine the fiscal impact of mining code proposals is critical and time-sensitive. The Burkinabé mining code is currently under revision, with a focus on corporate taxes, governance and environmental impacts, in consultation with mining companies. The authorities have requested technical assistance (TA) on mining taxation, a central TA priority.

through continued reforms of the cotton parastatal (MEFP, 9 39). They are also building on efforts to eliminate fraud and redundant payments to civil servants by setting up a system of biometric identification and using it to clean and harmonize the payroll and the civil service roster (MEFP, $₫$ 37).

\section{As a result of these efforts, the fiscal deficit (commitment basis, including} grants) will be reduced from 3.5 percent of GDP in 2012 to 3.0 percent in 2015. This deficit consolidation takes place despite implementation of the SCADD and reflects a conservative assumption that grants are assumed to decrease from 6.7 percent of GDP in 2012 to 5.8 percent in 2015. Domestic revenues are expected to increase from 16.1 percent of GDP in 2012 to 17.5 percent in 2015 , closing what has traditionally been a gap with regional peers. Underlying tax revenues are projected to increase to 
15.1 percent of GDP, closer to the WAEMU target of 17.0 percent. Despite implementation of the new spending for the new social protection policy, current spending will be reduced from about 14.3 percent in 2012 to around 13.1 percent in 2015, leaving space to increase domestically-financed investment from 6.3 percent to about 7.1 percent of GDP.

19. The authorities are maintaining the momentum of the PFM reform agenda to ensure that capacity constraints do not hinder the implementation of their development strategy (MEFP $\uparrow 14,15$, and 34). In addition to expenditure verification units in five line ministries to reduce delays in the chain of execution, they have introduced a number of checkpoints at different stages of the chain to increase accountability. They have also made steady progress toward budgeting for multi-year programs.

\section{Balance of Payments}

\section{Current account deterioration in 2012 reflects an increase in food import} needs, and higher international food and fuel prices. Current account projections were revised to take into account the food security crisis and fuel price developments, and the current account balance is therefore expected to deteriorate from 1.2 percent of GDP in 2011 to 4.7 percent in 2012. The deterioration would have been worse, but for higher projections for gold exports. The balance of payment projections have also been revised to reflect the fact that, after domestic wage and supplier payments, most gold export proceeds are not being repatriated to Burkina Faso, but rather are being held in overseas accounts and/or used to repay loans from parent companies abroad. The mission encouraged the BCEAO National Directorate to undertake additional balance of payments surveys to ensure that mining transactions are being fully and accurately reflected in the balance of payments and its forecasts. As a result, the 2012 forecast indicates a balance of payments need of CFAF 55.7 billion.

\section{Over the medium term, the current account is expected to improve with} strengthening mining exports. The main years of accelerated production and export are through 2016-17, and the forecast foresees some deterioration thereafter, mitigated by the diversification measures embodied in the SCADD.

\section{E. Financial Sector Policies}

22. Burkina's financial sector remains broadly sound. Despite continued strong credit growth in recent years, boosted mainly by trading sectors with nascent lending indirectly related to mining, the rate of nonperforming loans has been reduced from 7.1 percent at end-2009 to 3.0 percent at end-2011. As of December 31, 2011, WAEMU increased minimum capital requirements from CFAF 1 billion to CFAF 5 billion. Of 20 financial institutions, 5 encountered difficulties complying with the new requirement. Using internal capital and funds borrowed from other commercial banks, the state-owned 
"Burkina Fund for Social and Economic Development" took equity shares in the five banks as a bridge to securing private equity partners. Four of the five banks appear to be well-poised for this transition. Ownership and management questions surrounding the fifth bank need to be resolved prior to equity sales.

23. Increasing access to financial sector services is a key element of the authorities' development strategy (MEFP, $\uparrow 40$ and 41). To broaden access to financial services in line with SCADD objectives, the authorities plan to focus efforts on microfinance and postal company services. They are setting up bodies to implement an action plan to promote microfinance development and regulate the sector. In addition, the authorities intend to diversify and improve the quality of services of the state-owned postal company, whose wide geographical network plays an important role in providing proximate services.

\section{F. Debt Sustainability and External Borrowing}

\section{A joint Bank-Fund 2012 debt sustainability assessment (Annex) recommends that Burkina Faso's "high" risk of debt distress should be moved to "moderate."} The new assessment is based on end-2011 data rather than end-2010, and reflects the new assumptions for gold production and exports, as well as somewhat more conservative growth projections in the out-years. The December 2011 joint DSA showed a high risk of debt distress because one risk indicator, the baseline projections of net present value (NPV) of external debt-to-exports, breached the indicative threshold as of 2026, both under the baseline and the most extreme shock scenario. The 2012 assessment shows no breach of indicative debt distress thresholds under the baseline scenario or standardized stress tests. Higher exports enter directly in the denominator of the ratio, and they also reduce debt accumulation in the numerator by reducing the current account deficit. However, given the high dependency on gold, staff therefore constructed a customized scenario based on World Bank Commodities Group projections for gold prices, which are lower than WEO projections. The effect of this change on export values was approximated by extending the standardized export shock for three further years (201317). Even without altering production, under this scenario the debt distress threshold with respect to exports is breached. The change to a "moderate" risk rating is based on this country-specific stress test. However, this assessment is also based on unchanged indicative thresholds set for a "medium performer" under the World Bank's CPIA ratings.

\section{The authorities have expressed interest in considering other financing} options for large infrastructure projects. They are in the process of defining a framework law on public-private partnerships and have requested technical assistance in this area. Similarly, they would like to explore the possibility of borrowing on nonconcessional terms for large infrastructure projects - as yet unspecified - that would have high economic returns. The authorities are reforming the investment project 
selection process and have set up a centralized secretariat to ensure project selection is consistent with SCADD objectives. The authorities recognize the need to further strengthen debt management capacity. They have requested technical assistance to prepare a medium-term debt strategy and a workshop on debt sustainability analysis to train technicians to undertake regular DSAs (MEFP, $\uparrow$ 38). The 2012 DSA update assumes some shift to less concessional loan financing in the future.

\section{Program Issues}

\section{The authorities have requested an augmentation of access under the ECF} arrangement in the amount of 60 percent of quota (US\$55.4 million) to help meet balance of payment needs arising from the shocks. This would be disbursed in two tranches, 40 percent of quota together with this review and 20 percent of quota upon completion of the fifth program review (scheduled for December 2012). Frontloading the augmentation is proposed in light of large unmet financing needs arising immediately in the third quarter (July-September). This would come on top of access of 77 percent of quota for the current arrangement, bringing total program access to 137 percent of quota. Although beyond program norms, this level does not exceed global or exceptional access limits. ${ }^{3}$ Indicators of capacity to repay the Fund (Table 9) suggests good capacity for repayment, and the 2012 joint debt sustainability assessment suggests that an augmentation would not have an adverse impact on improved debt dynamics.

\section{The authorities and the mission agreed to move to a quantitative} performance criterion measuring net domestic financing instead of the deficit (Attachments I and II to the Minister's Letter of Intent). The mission suggested this change to remove ambiguity associated with cash adjustments to the deficit and external financing for projects. However, the former deficit criterion (with adjustors) is being retained as an indicative target for a transition period to allow comparisons over time. The change necessitates a request to modify the quantitative performance criterion for the program for end-June and end-December 2012, but a request would have been necessary using the deficit criterion in any case in light of expanded spending. The authorities and mission also agreed to other technical changes to streamline program monitoring to make it more transparent and relevant. The indicative target on social spending is being reduced from 100 to 90 percent of budget execution, since it currently cannot be met in the absence of overspending. This indicator tracks spending of ministries primarily in charge

\footnotetext{
${ }^{3}$ ECF norms are 75 percent of quota for countries with total outstanding concessional IMF credit under all facilities of between 100 and 200 percent of quota. Global annual and cumulative limits are 100 percent of quota per year and 300 percent of quota cumulatively across all concessional facilities, while exceptional access is 150 percent of quota annually and 450 percent of quota on a cumulative basis across all concessional facilities.
} 
of implementing the SCADD and social and SCADD-related spending of other ministries.

28. The main risks to the program are externalities associated with the political situation in Mali, including the influx of refugees. This would increase costs and import needs further, and put further pressure on the authorities to reprogram spending earmarked for the SCADD. Other risks include insufficient rainfall and no recovery in grain production and/or significant diversion of SCADD financing, which would affect the growth outlook. Another risk is that capacity constraints impede full implementation of the SCADD or effective handling of the refugee crisis. Domestic social unrest over higher fuel and food prices and spillover of the political turmoil in Mali remain everpresent risks. A less tangible risk is potential Dutch disease type effects from the rapidlyexpanding mining industry.

\section{Staff Appraisal}

29. The authorities have taken decisive adjustment measures to help meet additional needs stemming from multiple shocks. The authorities' decision to raise retail fuel prices to halve the implicit fuel subsidy and phase out the remainder going forward is particularly notable since domestic unrest less than a year ago was based on the "high cost of living." Moreover, their detailed and phased plan for addressing the food security crisis provides well-targeted relief, while minimizing waste. Measures to contain non-priority spending and good progress in enhancing revenue collection provide additional room for use of domestic resources.

30. The SCADD represents a proactive agenda to reduce vulnerability and increase resilience to future shocks. The SCADD has a number of specific near term measures to diversify agricultural production, sustain food production during adverse weather conditions, and improve food distribution. Measures to reduce factor costs, boost energy supplies, and improve the business environment are important complements to encourage diverse investment and reduce the country's vulnerability. Expansion of a well-articulated social safety net and employment schemes should also increase resilience.

31. New donor commitments have been made, but remaining financing needs are still significant. Donors made significant pledges to cover SCADD and about 40 percent of the costs of the food security program. However, the costs of the program are concentrated in the summer months, and more and faster external financing is needed. The Malian crisis has been evolving rapidly, and humanitarian aid providers are overwhelmed. The living conditions of the refugees are dire and deteriorating.

\section{A frontloaded augmentation of access under the ECF arrangement would} provide support for immediate import needs and should catalyze other financing. Augmentation of IMF program access will have an important effect in encouraging other 
large donors to act more decisively on Burkina Faso's behalf. Concern that augmentation would crowd out other more concessional sources of financing is diminished, since already identified remaining needs are not yet fully met, and the likelihood that those needs will increase further. A more probable outcome is that, even with new commitments by donors, the authorities will have to divert domestically-financed investment spending to cover immediate crisis needs.

33. While the food security program is well specified, the government needs to take a more prominent role in handling the unfolding refugee crisis. This would undoubtedly divert already scarce capacity from the SCADD and slow down its implementation, but may be necessary to stave off a more severe humanitarian crisis. Aside from the humanitarian imperative itself, events in Mali already pose security risks for the region and a crisis could intensify the risk of destabilization.

\section{Over the medium term, the authorities' commitment to implementation of the SCADD provides a good platform for continuing Burkina Faso's strong macroeconomic policy track record. The authorities wish to continue improving} revenue performance and containing non-priority spending to make more fiscal space for implementation of the SCADD. Development partners have expressed strong support for the SCADD's reform agenda as an effective means for improving Burkina Faso's resilience to shocks and hastening progress toward achieving the MDGs. The effectiveness of the SCADD in accelerating progress toward achieving the MDGs will require more determined action in some difficult areas, such as reducing transport costs through eliminating quotas and reforming the judiciary. It will also be important that infrastructure projects be carefully chosen to ensure cost-effectiveness, particularly with more debt financing.

\section{Staff supports completion of the fourth review and the authorities' request} for augmented access under the ECF arrangement, with frontloaded disbursement in two tranches. Staff also supports modification of Quantitative Performance Criteria (QPCs) for end-June and end-December 2012 to reflect higher crisis-related spending and new modalities for program monitoring. 
Table 1. Burkina Faso: Selected Economic and Financial Indicators, 2010-15

\begin{tabular}{|c|c|c|c|c|c|c|c|c|}
\hline & 2010 & 2011 & & 2012 & & 2013 & 2014 & 2015 \\
\hline & & Est. & Prog. ${ }^{2}$ & Prog. ${ }^{2,3}$ & Proj. & \multicolumn{3}{|c|}{ Proj. } \\
\hline & \multicolumn{8}{|c|}{ (Annual percentage change, unless otherwise indicated) } \\
\hline \multicolumn{9}{|l|}{ GDP and prices } \\
\hline GDP at constant prices & 7.9 & 4.2 & 5.8 & 5.8 & 7.0 & 7.0 & 7.0 & 7.0 \\
\hline GDP deflator & 2.8 & 5.6 & 2.2 & 2.2 & 3.5 & 2.0 & 2.0 & 2.0 \\
\hline Consumer prices (annual average) & -0.6 & 2.7 & 2.0 & 2.0 & 3.0 & 2.0 & 2.0 & 2.0 \\
\hline Consumer prices (end of period) & -0.3 & 5.1 & 2.0 & 2.0 & 3.0 & 2.0 & 2.0 & 2.0 \\
\hline \multicolumn{9}{|l|}{ Money and credit } \\
\hline Net domestic assets (banking system) ${ }^{1}$ & 11.6 & 12.5 & 11.0 & 11.0 & 13.1 & 11.2 & 12.4 & 11.5 \\
\hline Credit to the government (banking system) ${ }^{1}$ & 6.4 & -1.9 & 0.0 & 0.0 & 2.5 & 3.6 & 1.9 & 1.1 \\
\hline Credit to the private sector ${ }^{1}$ & 8.9 & 13.6 & 11.0 & 11.0 & 10.6 & 7.5 & 10.5 & 10.4 \\
\hline Broad money (M3) & 19.1 & 12.8 & 13.6 & 13.6 & 10.8 & 10.8 & 12.4 & 11.9 \\
\hline \multicolumn{9}{|l|}{ External sector } \\
\hline Exports (f.o.b.; valued in CFA francs) & 84.7 & 37.5 & 11.1 & 11.1 & 18.4 & 15.0 & 15.0 & 8.8 \\
\hline Imports (f.o.b.; valued in CFA francs) & 30.8 & 26.3 & 15.4 & 15.4 & 26.0 & 7.9 & 10.6 & 7.7 \\
\hline Terms of trade & -1.2 & 10.2 & 6.4 & 6.4 & 3.2 & 2.0 & 3.5 & 2.5 \\
\hline Real effective exchange rate $(-=$ depreciation) & -8.4 & 1.6 & $\ldots$ & $\ldots$ & $\ldots$ & $\ldots$ & $\ldots$ & $\cdots$ \\
\hline \multirow[t]{2}{*}{ CFAF/US\$ (annual average) } & 487.5 & 471.4 & $\cdots$ & $\cdots$ & $\cdots$ & $\cdots$ & $\cdots$ & $\cdots$ \\
\hline & \multicolumn{8}{|c|}{ (Percent of GDP, unless otherwise indicated) } \\
\hline \multicolumn{9}{|l|}{ Central government finances } \\
\hline Current revenue & 15.6 & 16.5 & 16.2 & 15.5 & 16.1 & 16.3 & 17.0 & 17.5 \\
\hline Of which: tax revenue & 12.9 & 14.5 & 14.1 & 13.5 & 14.0 & 14.2 & 14.8 & 15.1 \\
\hline Total expenditure and net lending & 24.6 & 24.3 & 26.1 & 25.0 & 26.7 & 26.0 & 26.2 & 26.3 \\
\hline Of which: current expenditure & 12.1 & 13.1 & 12.8 & 12.2 & 14.3 & 12.8 & 13.0 & 13.0 \\
\hline Overall fiscal balance, excl. grants (commitmer & -9.1 & -7.7 & -9.9 & -9.5 & -10.5 & -9.7 & -9.1 & -8.8 \\
\hline Overall fiscal balance, incl. grants (commitmen & -4.5 & -2.5 & -3.0 & -2.9 & -3.5 & -3.3 & -3.0 & -3.0 \\
\hline \multicolumn{9}{|l|}{ Savings and investment } \\
\hline Current account balance (incl official transfers) & -2.3 & -1.2 & -6.9 & -6.6 & -4.4 & -2.9 & -2.8 & -2.7 \\
\hline Gross investment & 18.3 & 15.6 & 16.4 & 15.7 & 18.4 & 16.7 & 15.4 & 15.5 \\
\hline Government & 7.8 & 7.1 & 9.4 & 9.0 & 8.7 & 9.2 & 9.3 & 9.3 \\
\hline Private & 10.6 & 8.5 & 7.0 & 6.7 & 9.7 & 7.5 & 6.1 & 6.2 \\
\hline Gross national savings & 16.0 & 14.4 & 9.6 & 9.2 & 14.0 & 13.8 & 12.6 & 12.8 \\
\hline Government & 7.8 & 9.3 & 7.0 & 6.7 & 5.6 & 6.7 & 7.2 & 7.4 \\
\hline Private & 8.2 & 5.1 & 2.6 & 2.5 & 8.4 & 7.1 & 5.5 & 5.4 \\
\hline \multicolumn{9}{|l|}{ Debt indicators } \\
\hline External debt & 23.9 & 24.1 & 23.4 & 22.4 & 24.0 & 23.8 & 23.9 & 24.1 \\
\hline NPV of external debt & $\ldots$ & 14.6 & 17.1 & 16.3 & 14.5 & 14.4 & 14.5 & 14.6 \\
\hline NPV of external debt (percent of exports) & $\ldots$ & 56.6 & 69.4 & 69.4 & 53.6 & 50.8 & 48.7 & 49.2 \\
\hline \multicolumn{9}{|l|}{ Memorandum items: } \\
\hline Nominal GDP (CFAF billions) & 4,371 & 4,807 & 5,092 & 5,326 & 5,326 & 5,813 & 6,345 & 6,925 \\
\hline Nominal GDP per capita (US\$) & 544 & 605 & $\ldots$ & $\ldots$ & $\ldots$ & $\ldots$ & $\ldots$ & $\ldots$ \\
\hline \multicolumn{9}{|c|}{ Sources: Burkinabè authorities; and IMF staff estimates and projections. } \\
\hline \multicolumn{9}{|c|}{${ }^{1}$ Percent of beginning-of-period broad money. } \\
\hline \multicolumn{9}{|l|}{2 Third ECF Review } \\
\hline Jsing current GDP projection as de & & & & & & & & \\
\hline
\end{tabular}


Table 2a. Burkina Faso: Consolidated Operations of the Central Government, 2010-15

\begin{tabular}{|c|c|c|c|c|c|c|c|c|}
\hline & \multirow[t]{2}{*}{2010} & \multicolumn{2}{|c|}{2011} & \multicolumn{2}{|c|}{2012} & \multirow[t]{2}{*}{2013} & \multirow{2}{*}{$\begin{array}{c}2014 \\
\text { Proj. }\end{array}$} & \multirow[t]{2}{*}{2015} \\
\hline & & Prog. ${ }^{7}$ & Prel. & Prog. ${ }^{7}$ & Proj. & & & \\
\hline & \multicolumn{8}{|c|}{ (CFAF billions) } \\
\hline Total revenue and grants & 880.2 & 1041.7 & 1047.3 & 1176.7 & 1234.8 & 1321.9 & 1469.7 & 1613.3 \\
\hline Total revenue & 681.3 & 711.7 & 793.6 & 826.0 & 858.7 & 948.8 & 1081.7 & 1210.5 \\
\hline Tax revenue & 565.7 & 614.4 & 695.6 & 719.2 & 745.3 & 827.9 & 941.8 & 1048.9 \\
\hline Income and profits ${ }^{1}$ & 133.6 & 158.2 & 201.0 & 181.4 & 191.7 & 216.3 & 250.0 & 282.5 \\
\hline Domestic goods and services & 318.4 & 337.9 & 366.8 & 401.8 & 410.9 & 453.1 & 501.1 & 548.0 \\
\hline International trade & 96.8 & 101.1 & 112.0 & 116.9 & 125.2 & 139.3 & 162.9 & 173.7 \\
\hline Other & 17.0 & 17.1 & 15.9 & 19.0 & 17.5 & 19.2 & 27.9 & 44.8 \\
\hline Nontax revenue ${ }^{2}$ & 115.5 & 97.4 & 98.0 & 106.9 & 113.4 & 121.0 & 139.8 & 161.6 \\
\hline Of which: royalties from gold & 12.1 & $\ldots$ & 17.7 & 39.7 & 45.2 & 53.1 & 62.8 & 68.8 \\
\hline Grants & 198.9 & 330.0 & 253.7 & 350.6 & 376.1 & 373.0 & 388.1 & 402.8 \\
\hline Project & 51.1 & 130.4 & 73.1 & 217.2 & 215.5 & 226.5 & 237.7 & 249.1 \\
\hline Program & 147.8 & 199.6 & 180.6 & 133.5 & 160.5 & 146.5 & 150.4 & 153.7 \\
\hline Of which: unidentified donor financing & 0.0 & 0.0 & 0.0 & 0.0 & 18.3 & 0.0 & 0.0 & 0.0 \\
\hline Expenditure and net lending ${ }^{3}$ & 1077.0 & 1225.5 & 1166.0 & 1330.2 & 1419.5 & 1510.9 & 1659.2 & 1819.3 \\
\hline Current expenditure & 530.9 & 635.2 & 628.3 & 650.3 & 761.3 & 742.9 & 822.0 & 898.5 \\
\hline Wages and salaries & 245.8 & 269.3 & 281.4 & 319.8 & 319.9 & 349.1 & 381.0 & 415.9 \\
\hline Goods and services & 90.8 & 104.4 & 97.1 & 104.0 & 104.0 & 119.3 & 136.6 & 150.5 \\
\hline Interest payments & 21.4 & 23.4 & 28.3 & 20.5 & 20.6 & 24.5 & 25.2 & 26.1 \\
\hline Domestic & 11.2 & 12.2 & 15.9 & 12.2 & 12.3 & 13.4 & 13.3 & 13. 1 \\
\hline External & 10.2 & 11.3 & 12.4 & 8.4 & 8.4 & 11.1 & 11.9 & 13.0 \\
\hline Current transfers & 172.9 & 238.1 & 221.4 & 206.0 & 316.8 & 250.0 & 279.2 & 306.1 \\
\hline \multirow{5}{*}{$\begin{array}{l}\text { Of which: } \\
\text { contingency expenditure } \\
\text { election } \\
\text { refugee } \\
\text { subsidies to SONABHY } \\
\text { subsidies for cotton input }\end{array}$} & 0.0 & 17.6 & 17.6 & 18.8 & 75.2 & 0.0 & 0.0 & 0.0 \\
\hline & $\ldots$ & $\ldots$ & $\ldots$ & $\ldots$ & 19.0 & $\ldots$ & $\ldots$ & $\ldots$ \\
\hline & $\ldots$ & $\ldots$ & $\ldots$ & 0.0 & 10.7 & $\ldots$ & $\ldots$ & $\ldots$ \\
\hline & $\ldots$ & $\ldots$ & 4.3 & 0.0 & 15.5 & $\ldots$ & $\ldots$ & $\ldots$ \\
\hline & $\ldots$ & $\ldots$ & $\ldots$ & 3.0 & 10.0 & $\ldots$ & $\ldots$ & $\ldots$ \\
\hline Investment expenditure & 484.7 & 596.6 & 488.6 & 686.8 & 665.1 & 763.9 & 839.2 & 922.8 \\
\hline Domestically financed & 280.8 & 301.7 & 286.4 & 329.6 & 333.5 & 402.0 & 444.1 & 491.6 \\
\hline Of which: election & & & & 11.0 & 11.0 & & & \\
\hline Externally financed ${ }^{5}$ & 203.9 & 295.0 & 202.2 & 357.2 & 331.6 & 362.0 & 395.0 & 431.2 \\
\hline \multirow{3}{*}{$\begin{array}{l}\text { Externally financed }{ }^{5} \\
\text { Of wich: contingency expenditure }{ }^{4} \\
\text { refugee }\end{array}$} & 0.0 & 17.6 & 17.6 & 0.0 & 36.9 & 0.0 & 0.0 & 0.0 \\
\hline & $\ldots$ & $\ldots$ & $\ldots$ & 0.0 & 2.3 & $\ldots$ & $\ldots$ & $\ldots$ \\
\hline & -6.2 & -6.3 & 2.7 & -6.9 & -6.9 & 4.0 & -2.0 & -2.0 \\
\hline Overall balance (commitment basis) & -196.8 & -183.8 & -118.8 & -153.5 & -184.7 & -189.0 & -189.4 & -206.0 \\
\hline \multirow{2}{*}{$\begin{array}{l}\text { Excluding unidentified donor financing } \\
\text { grants }\end{array}$} & -196.8 & -183.8 & -118.8 & -153.5 & -203.1 & -189.0 & -189.4 & -206.0 \\
\hline & -395.7 & -513.8 & -372.4 & -504.1 & -560.8 & -562.0 & -577.5 & -608.8 \\
\hline Cash basis adjustment ${ }^{6}$ & -2.1 & -23.3 & 12.9 & -0.5 & 0.0 & -0.5 & -0.5 & -0.5 \\
\hline Overall balance (cash basis) & -198.9 & -207.1 & -105.8 & -154.0 & -184.7 & -189.5 & -189.9 & -206.5 \\
\hline Errors and omissions & 4.0 & 0.0 & 5.8 & 0.0 & 0.0 & 0.0 & 0.0 & 0.0 \\
\hline Financing & 194.9 & 197.5 & 100.0 & 144.4 & 147.3 & 184.6 & 189.9 & 206.5 \\
\hline Foreign financing & 150.7 & 139.0 & 109.0 & 118.8 & 94.9 & 109.4 & 130.2 & 153.8 \\
\hline Drawings & 165.4 & 164.6 & 129.1 & 140.0 & 116.1 & 135.4 & 157.3 & 182.1 \\
\hline Project loans & 152.8 & 164.6 & 129.1 & 140.0 & 116.1 & 135.4 & 157.3 & 182.1 \\
\hline Program loans & 12.6 & 0.0 & 0.0 & 0.0 & 0.0 & 0.0 & 0.0 & 0.0 \\
\hline Amortization (excl. IMF) & -14.7 & -25.6 & -20.1 & -21.2 & -21.2 & -26.0 & -27.2 & -28.3 \\
\hline Domestic financing & 44.2 & 58.6 & -9.0 & 25.6 & 52.4 & 75.2 & 59.7 & 52.7 \\
\hline Bank financing & 70.5 & 36.4 & -24.6 & 46.3 & 0.0 & 55.2 & 34.7 & 22.7 \\
\hline Central bank & 18.2 & 40.7 & -31.4 & 46.3 & 0.0 & 0.0 & 0.0 & 0.0 \\
\hline Commercial banks & 52.3 & -4.3 & 6.9 & 0.0 & 0.0 & 55.2 & 34.7 & 22.7 \\
\hline Nonbank financing & -26.3 & 22.2 & 15.6 & -20.7 & 52.4 & 20.0 & 25.0 & 30.0 \\
\hline Of which government securities ${ }^{6}$ & -5.0 & 22.2 & 4.6 & -20.7 & 52.4 & 20.0 & 25.0 & 30.0 \\
\hline Financing gap & 0.0 & 9.6 & 0.0 & 9.6 & 37.4 & 4.8 & 0.0 & 0.0 \\
\hline Of which: IMF future disbursements & 0.0 & 9.6 & 0.0 & 9.6 & 37.4 & 4.8 & 0.0 & 0.0 \\
\hline \multicolumn{9}{|l|}{ Memorandum items : } \\
\hline Poverty-reducing expenditure & 297.2 & 314.4 & 298.2 & 384.6 & 391.2 & 412.7 & 469.5 & 484.7 \\
\hline
\end{tabular}

Sources: Burkinabè authorities; and IMF staff es timates and projections.

${ }^{1}$ Includes for 2011 about 0.7 percent of GDP of provisional payments for the new corporate income tax (IS), which is a one-time increase in tax collection.

${ }^{2}$ Includes for 2010 and 2011 exceptional revenue from renewal of mobile licence.

${ }^{3}$ Commitment ("engagement") basis.

${ }^{4}$ Contingency expenditure to address the impact of exogenous shocks, notably the Sahel drought in 2012.

${ }^{5}$ Includes for 2012 spending for the food insecurity and refugees from Mali, which will be reclassified into current transfers once executed.

${ }^{6}$ Does not include for 2012 the 10 -year bond is sued to SONABHY to repay implicit subsidies for petroleum products in 2011 (for a total of CFAF 38.3 billion); its amortization is reflected in nonbank financing from 2013.

7 Third ECF Review. 
Table 2b. Burkina Faso: Consolidated Operations of the Central Government, 2010-15

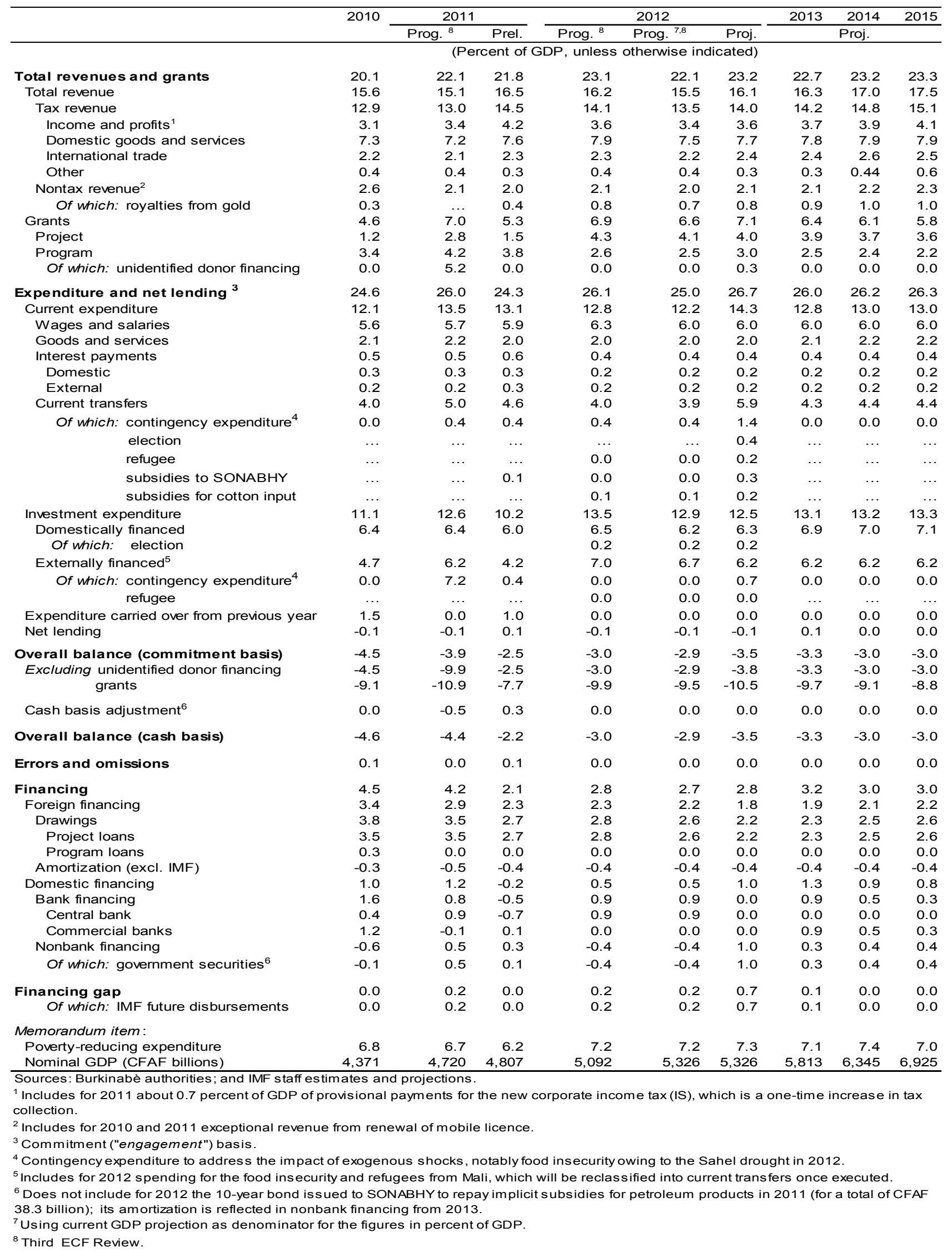


Table 3. Burkina Faso: Monetary Survey, 2010-15

\begin{tabular}{|c|c|c|c|c|c|c|c|}
\hline & \multirow[t]{2}{*}{2010} & \multirow[t]{2}{*}{2011} & \multicolumn{2}{|c|}{2012} & 2013 & 2014 & 2015 \\
\hline & & & Prog. ${ }^{1}$ & Proj. & \multicolumn{3}{|c|}{ Proj. } \\
\hline & \multicolumn{7}{|c|}{ (CFAF billions) } \\
\hline Net foreign assets & 647.6 & 652.1 & 854.2 & 617.9 & 611.4 & 612.4 & 620.3 \\
\hline BCEAO & 300.8 & 245.9 & 481.1 & 211.7 & 205.2 & 206.2 & 214.1 \\
\hline Assets & 525.6 & 450.1 & 684.3 & 412.3 & 401.8 & 397.3 & 394.3 \\
\hline Liabilities & 224.8 & 204.2 & 203.2 & 200.6 & 196.6 & 191.1 & 180.3 \\
\hline Commercial banks & 346.8 & 406.2 & 373.2 & 406.2 & 406.2 & 406.2 & 406.2 \\
\hline Net domestic assets & 671.9 & 836.4 & 931.3 & 1031.1 & 1215.6 & 1441.4 & 1677.2 \\
\hline Net domestic credit & 787.3 & 943.5 & 992.9 & 1138.3 & 1322.7 & 1548.5 & 1784.4 \\
\hline Net credit to government & 16.7 & -7.9 & 6.0 & 29.5 & 89.5 & 124.3 & 147.1 \\
\hline Treasury & 16.5 & 38.6 & 6.3 & 76.0 & 136.0 & 170.7 & 193.6 \\
\hline BCEAO & -27.0 & 11.2 & -12.8 & 48.6 & 53.4 & 53.4 & 53.4 \\
\hline Commercial banks & 43.5 & 27.4 & 19.1 & 27.4 & 82.6 & 117.3 & 140.2 \\
\hline Other central government & 0.2 & -46.5 & -0.3 & -46.5 & -46.5 & -46.5 & -46.5 \\
\hline Credit to the economy & 770.6 & 951.4 & 986.9 & 1108.7 & 1233.2 & 1424.2 & 1637.3 \\
\hline Crop credit & 10.3 & 14.7 & 12.7 & 17.3 & 19.3 & 22.4 & 25.9 \\
\hline Other & 789.2 & 936.7 & 974.2 & 1091.5 & 1213.9 & 1401.8 & 1611.4 \\
\hline Other items (net) & -115.4 & -107.1 & -61.6 & -107.1 & -107.1 & -107.1 & -107.1 \\
\hline Broad money & 1319.5 & 1488.5 & 1785.5 & 1649.0 & 1827.0 & 2053.8 & 2297.5 \\
\hline \multirow[t]{2}{*}{ Of which: Priv. deposits in comm. banks } & 1029.2 & 1224.6 & 1444.6 & 1404.9 & 1562.0 & 1762.3 & 1977.6 \\
\hline & \multicolumn{7}{|c|}{$\begin{array}{c}\text { (Annual changes in } \% \text { of broad money from } 12 \text { months earlier, unless } \\
\text { otherwise indicated) }\end{array}$} \\
\hline \multicolumn{8}{|l|}{ Memorandum items: } \\
\hline Net foreign assets & 7.6 & 0.3 & 2.6 & -2.3 & -0.4 & 0.1 & 0.4 \\
\hline Net domestic assets & 11.6 & 12.5 & 11.0 & 13.1 & 11.2 & 12.4 & 11.5 \\
\hline Net credit to government & 6.4 & -1.9 & 0.0 & 2.5 & 3.6 & 1.9 & 1.1 \\
\hline Credit to the private sector & 8.9 & 13.7 & 11.0 & 10.6 & 7.5 & 10.5 & 10.4 \\
\hline (annual percentage change) & 14.7 & 23.5 & 21.3 & 16.5 & 11.2 & 15.5 & 15.0 \\
\hline Money supply & 19.1 & 12.8 & 13.6 & 10.8 & 10.8 & 12.4 & 11.9 \\
\hline Of which: bank deposits & 21.7 & 14.8 & 18.2 & 12.1 & 9.5 & 11.0 & 10.5 \\
\hline Currency velocity (GDP/broad money) & 3.3 & 3.2 & 2.9 & 3.2 & 3.2 & 3.1 & 3.0 \\
\hline
\end{tabular}

Sources: Burkinabè authorities; and IMF staff estimates and projections.

${ }^{1}$ Third ECF Review. 
Table 4. Burkina Faso: Balance of Payments, 2010-15

\begin{tabular}{|c|c|c|c|c|c|c|c|}
\hline & \multirow[t]{2}{*}{2010} & \multirow{2}{*}{$\begin{array}{r}2011 \\
\text { Prel. } \\
\end{array}$} & \multicolumn{2}{|c|}{2012} & 2013 & 2014 & 2015 \\
\hline & & & Prog. ${ }^{2}$ & Proj. & \multicolumn{3}{|c|}{ Proj. } \\
\hline & \multicolumn{7}{|c|}{ (CFAF billions) } \\
\hline Current account & -101.7 & -59.6 & -349.2 & -234.6 & -170.8 & -175.4 & -185.7 \\
\hline Excluding official transfers & -271.8 & -262.9 & -505.9 & -418.4 & -341.0 & -349.9 & -364.1 \\
\hline Trade balance & -68.9 & 0.6 & -196.0 & -81.1 & 3.3 & 67.7 & 90.7 \\
\hline Exports of goods & 785.2 & 1079.5 & 1150.6 & 1278.3 & 1470.6 & 1690.7 & 1838.7 \\
\hline Of which: cotton & 113.5 & 130.9 & 175.8 & 173.4 & 192.1 & 198.5 & 206.1 \\
\hline gold & 512.0 & 767.6 & 799.3 & 904.5 & 1061.6 & 1256.5 & 1375.3 \\
\hline Imports of goods & -854.2 & -1079.0 & -1346.6 & -1359.4 & -1467.3 & -1623.0 & -1748.0 \\
\hline Of which: oil & -220.4 & -308.6 & -360.2 & -380.2 & -397.5 & -406.5 & -408.5 \\
\hline Of which: food & -68.1 & -84.3 & -86.6 & -153.7 & -99.1 & -110.4 & -120.9 \\
\hline Of which: public investment & -387.7 & -390.9 & -549.4 & -532.1 & -611.1 & -671.3 & -738.2 \\
\hline Services, net & -261.6 & -328.4 & -382.4 & -407.4 & -417.5 & -493.9 & -533.8 \\
\hline Exports of services & 150.4 & 156.7 & 104.2 & 167.7 & 179.4 & 192.0 & 209.4 \\
\hline Imports of services & -412.1 & -485.2 & -486.7 & -575.1 & -596.9 & -685.9 & -743.2 \\
\hline Of which: freight and insurance & -195.7 & -247.1 & -312.9 & -311.4 & -336.1 & -371.8 & -400.4 \\
\hline Income, net & -3.2 & 0.2 & 8.5 & 6.1 & 7.8 & 9.7 & 11.1 \\
\hline Of which: interest on public debt (incl. IMF) & -10.2 & -11.2 & -12.4 & -8.4 & -11.1 & -11.9 & -13.0 \\
\hline Current transfers & 232.0 & 268.1 & 220.7 & 247.8 & 235.5 & 241.2 & 246.3 \\
\hline Private transfers, net & 61.9 & 64.8 & 64.0 & 64.0 & 65.3 & 66.6 & 67.9 \\
\hline Official transfers, net & 170.2 & 203.4 & 156.7 & 183.8 & 170.2 & 174.5 & 178.3 \\
\hline Of which: program grants & 147.8 & 180.6 & 133.5 & 160.5 & 146.5 & 150.4 & 153.7 \\
\hline Of which: unidentified donor financing & 0.0 & 0.0 & 0.0 & 18.3 & 0.0 & 0.0 & 0.0 \\
\hline Capital account & 98.8 & 121.2 & 244.0 & 264.0 & 275.0 & 286.2 & 297.6 \\
\hline Project grants & 51.1 & 73.1 & 217.2 & 215.5 & 226.5 & 237.7 & 249.1 \\
\hline Financial account & 75.6 & -31.1 & 145.4 & -63.6 & -110.8 & -109.8 & -103.9 \\
\hline Direct investment & 16.8 & 17.9 & 6.6 & 18.0 & 23.0 & 25.0 & 27.3 \\
\hline Portfolio investment & 15.8 & 9.1 & 10.0 & 10.0 & 10.0 & 10.0 & 10.0 \\
\hline Other investment & 43.1 & -58.1 & 128.8 & -91.6 & -143.8 & -144.8 & -141.2 \\
\hline Long-term investment & 40.7 & -59.7 & 123.8 & -96.6 & -148.8 & -149.8 & -146.2 \\
\hline Project loans & 152.8 & 129.1 & 140.0 & 116.1 & 135.4 & 157.3 & 182.1 \\
\hline Program loans & 12.6 & 0.0 & 0.0 & 0.0 & 0.0 & 0.0 & 0.0 \\
\hline Amortization of public loans (excl. IMF) & -14.7 & -20.1 & -21.2 & -21.2 & -26.0 & -27.2 & -28.3 \\
\hline Other private & -110.0 & -168.7 & 5.0 & -191.5 & -258.2 & -280.0 & -300.0 \\
\hline Short-term investment & 2.4 & 1.6 & 5.0 & 5.0 & 5.0 & 5.0 & 5.0 \\
\hline Errors and ommisions/gap & 6.5 & -26.0 & 0.0 & 0.0 & 0.0 & 0.0 & 0.0 \\
\hline Overall balance & 79.3 & 4.5 & 40.2 & -34.2 & -6.5 & 1.0 & 7.9 \\
\hline Financing & -79.3 & -4.5 & -49.8 & -3.2 & 1.7 & -1.0 & -7.9 \\
\hline Net change in foreign assets of the central bank & 89.8 & 54.9 & -49.8 & -3.2 & 1.7 & -1.0 & -7.9 \\
\hline Of which: gross official reserves & 63.1 & 75.5 & -46.3 & 0.4 & 5.7 & 4.5 & 3.0 \\
\hline IMF net financing & 5.9 & 8.6 & -3.5 & -3.6 & -3.9 & -5.5 & -10.9 \\
\hline Uses of resources & 5.9 & 9.6 & 0.0 & 0.0 & 0.0 & 0.0 & 0.0 \\
\hline Repayments (excluding charges) & 0.0 & -1.0 & -3.5 & -3.6 & -3.9 & -5.5 & -10.9 \\
\hline Net foreign assets of commercial banks & -169.0 & -59.4 & 0.0 & 0.0 & 0.0 & 0.0 & 0.0 \\
\hline \multirow[t]{2}{*}{ Financing Gap ${ }^{1}$} & 0.0 & 0.0 & 9.6 & 37.4 & 4.8 & 0.0 & 0.0 \\
\hline & \multicolumn{7}{|c|}{ (Percent of GDP, unless otherwise indicated) } \\
\hline Memorandum items: & & & & & & & \\
\hline Trade balance $(-=$ deficit $)$ & -1.6 & 0.0 & -3.8 & -1.5 & 0.1 & 1.1 & 1.3 \\
\hline Gold export volume (metric tons) & 23.1 & 32.4 & 30.6 & 35.0 & 40.0 & 46.1 & 49.1 \\
\hline Current account $(-=$ deficit $)$ & -2.3 & -1.2 & -6.9 & -4.4 & -2.9 & -2.8 & -2.7 \\
\hline Gross official reserves (imputed reserves, bns US\$) & 1.1 & 0.9 & 1.5 & 0.8 & 0.8 & 0.8 & 0.8 \\
\hline GDP at current prices (CFAF billions) & 4,371 & 4,807 & 5,092 & 5,326 & 5,813 & 6,345 & 6,925 \\
\hline
\end{tabular}

Sources: Burkinabè authorities; and IMF staff estimates and projections.

${ }^{1}$ Prospective IMF disbursements, including proposed augmentation.

2 Third ECF Review. 
Table 5. Burkina Faso: Financing Requirements, 2011-14

(CFAF billions, unless otherwise indicated)

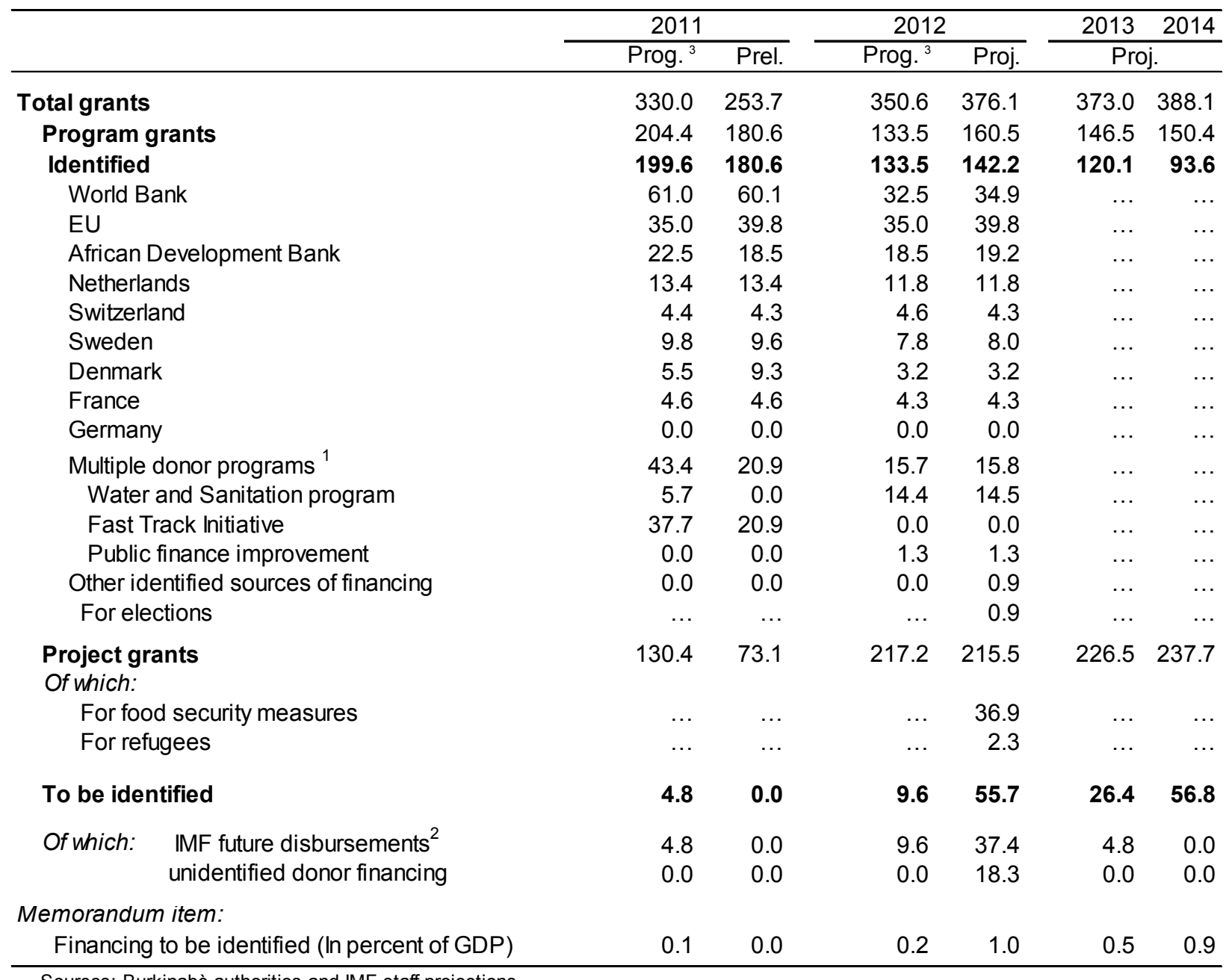

Sources: Burkinabè authorities and IMF staff projections.

${ }^{1}$ Managed by the World Bank.

${ }^{2}$ Including an augmentation of access of CFAF 27.8 billion.

${ }^{3}$ Third ECF Review. 
Table 6. Burkina Faso: Quantitative Assessment Criteria and Indicative Targets, 2011

(CFAF billions, cumulative from beginning of year; unless otherwise indicated)

\begin{tabular}{|c|c|c|c|c|c|c|c|c|}
\hline & \multirow{2}{*}{\multicolumn{4}{|c|}{2011}} & \multirow{2}{*}{\multicolumn{4}{|c|}{ Dec. $^{6}$}} \\
\hline & & & & & & & & \\
\hline & Prog. ${ }^{7}$ & Adj. & Act. & Status & Prog. ${ }^{7}$ & Adj. & Act. & Status \\
\hline \multicolumn{9}{|l|}{ Quantitative Assessment Criteria } \\
\hline Ceiling on the overall fiscal deficit including grants ${ }^{1}$ & 178.3 & 218.3 & 107.0 & Met & 186.1 & 211.1 & 117.4 & Met \\
\hline $\begin{array}{l}\text { Ceiling on the amount of new nonconcessional external debt contracte } \\
\text { guaranteed by the government } 2,3\end{array}$ & 0.0 & $\ldots$ & 0.0 & Met & 0.0 & $\ldots$ & 0.0 & Met \\
\hline $\begin{array}{l}\text { Ceiling on the amount of new external debt of less than one year's ma } \\
\text { contracted or guaranteed by the government } 2,3\end{array}$ & 0.0 & $\ldots$ & 0.0 & Met & 0.0 & $\ldots$ & 0.0 & Met \\
\hline Accumulation of external arrears ${ }^{2}$ & 0.0 & $\ldots$ & 0.0 & Met & 0.0 & $\ldots$ & 0.0 & Met \\
\hline \multicolumn{9}{|l|}{ Indicative targets } \\
\hline Government revenue & 508.2 & $\ldots$ & 580.7 & Met & 711.7 & $\ldots$ & 793.6 & Met \\
\hline Poverty-reducing social expenditure & 229.5 & $\ldots$ & 206.1 & Not met & 314.4 & $\ldots$ & 298.2 & Not met \\
\hline Large taxpayer non-filer rate ${ }^{4}$ & 5.0 & $\ldots$ & 2.0 & Met & 5.0 & $\ldots$ & 3.0 & Met \\
\hline Accumulation of new domestic arrears & 0.0 & $\ldots$ & 0.0 & Met & 0.0 & $\ldots$ & 0.0 & Met \\
\hline \multicolumn{9}{|c|}{ Maximum upward adjustment of deficit ceiling including grants owing to: } \\
\hline Shortfall in grants relative to program projections & 25.0 & $\ldots$ & 25.0 & $\ldots$ & 25.0 & $\ldots$ & 25.0 & $\ldots$ \\
\hline Excess in concessional loan financing relative to program projections & 15.0 & $\ldots$ & 15.0 & $\ldots$ & 15.0 & $\ldots$ & 0.0 & $\ldots$ \\
\hline \multicolumn{9}{|l|}{ Adjustment factors } \\
\hline Shortfall in grants relative to program projections & 0.0 & $\ldots$ & 46.1 & $\ldots$ & 0.0 & $\ldots$ & 76.3 & $\ldots$ \\
\hline Excess in concessional loan financing relative to program projections & 0.0 & $\ldots$ & 18.1 & $\ldots$ & 0.0 & $\ldots$ & -35.5 & $\ldots$ \\
\hline \multicolumn{9}{|l|}{ Memorandum items: } \\
\hline Basic primary balance (cash basis) & -196.9 & $\ldots$ & -79.3 & $\ldots$ & -218.7 & $\ldots$ & -129.0 & $\ldots$ \\
\hline Grants & 195.2 & $\ldots$ & 149.2 & $\ldots$ & 330.0 & $\ldots$ & 253.7 & $\ldots$ \\
\hline Concessional loans & 82.3 & $\ldots$ & 100.4 & $\ldots$ & 164.6 & $\ldots$ & 129.1 & $\ldots$ \\
\hline
\end{tabular}

Sources: Burkinabè authorities; and IMF staff estimates and projections.

1 The ceiling on the overall fiscal deficit is to be adjusted in line with the Technical Memorandum of Understanding definition. It is calculated on a commitment basis.

2 To be observed continuously.

${ }^{3}$ Excluding treasury bills and bonds issued in CFA francs on the regional West African Economic and Monetary Union (WAEMU) market. This ceiling

excludes supplier credit with a maturity of one year or less.

${ }^{4}$ Maximum \% share of late/nonfilers to the Large Taxpayer Office. Applies to average over respective quarter. The value for December corresponds to the fourth quarter.

${ }^{5}$ Indicative target.

${ }^{6}$ Quantitative performance criterion.

${ }^{7}$ Third ECF Review. 
Table 7. Structural Benchmarks, December 2011

\begin{tabular}{|l|l|l|}
\hline $\begin{array}{l}\text { Implement a new pricing and taxation } \\
\text { system for petroleum products. }\end{array}$ & End-Dec. 2011 & Met with delay \\
\hline $\begin{array}{l}\text { Set up a one-stop window for customs } \\
\text { clearance. }\end{array}$ & End-Dec. 2011 & Met. \\
\hline $\begin{array}{l}\text { Set up an electronic system to improve } \\
\text { merchandise valuation in two customs } \\
\text { posts. }\end{array}$ & End-Dec. 2011 & Met. \\
\hline $\begin{array}{l}\text { Complete the civil service survey and } \\
\text { harmonize the payroll and the civil } \\
\text { service roster. }\end{array}$ & End-Dec. 2011 & Met. \\
\hline $\begin{array}{l}\text { Adopt and put in place the microfinance } \\
\text { strategy. }\end{array}$ & End-Dec. 2011 & Partly met with delay. \\
\hline $\begin{array}{l}\text { Establish expenditure verification units in } \\
\text { five ministries }\end{array}$ & March 2012 & Met. \\
\hline
\end{tabular}


Table 8. Burkina Faso: Poverty-Reducing Social Expenditure, 2005-12

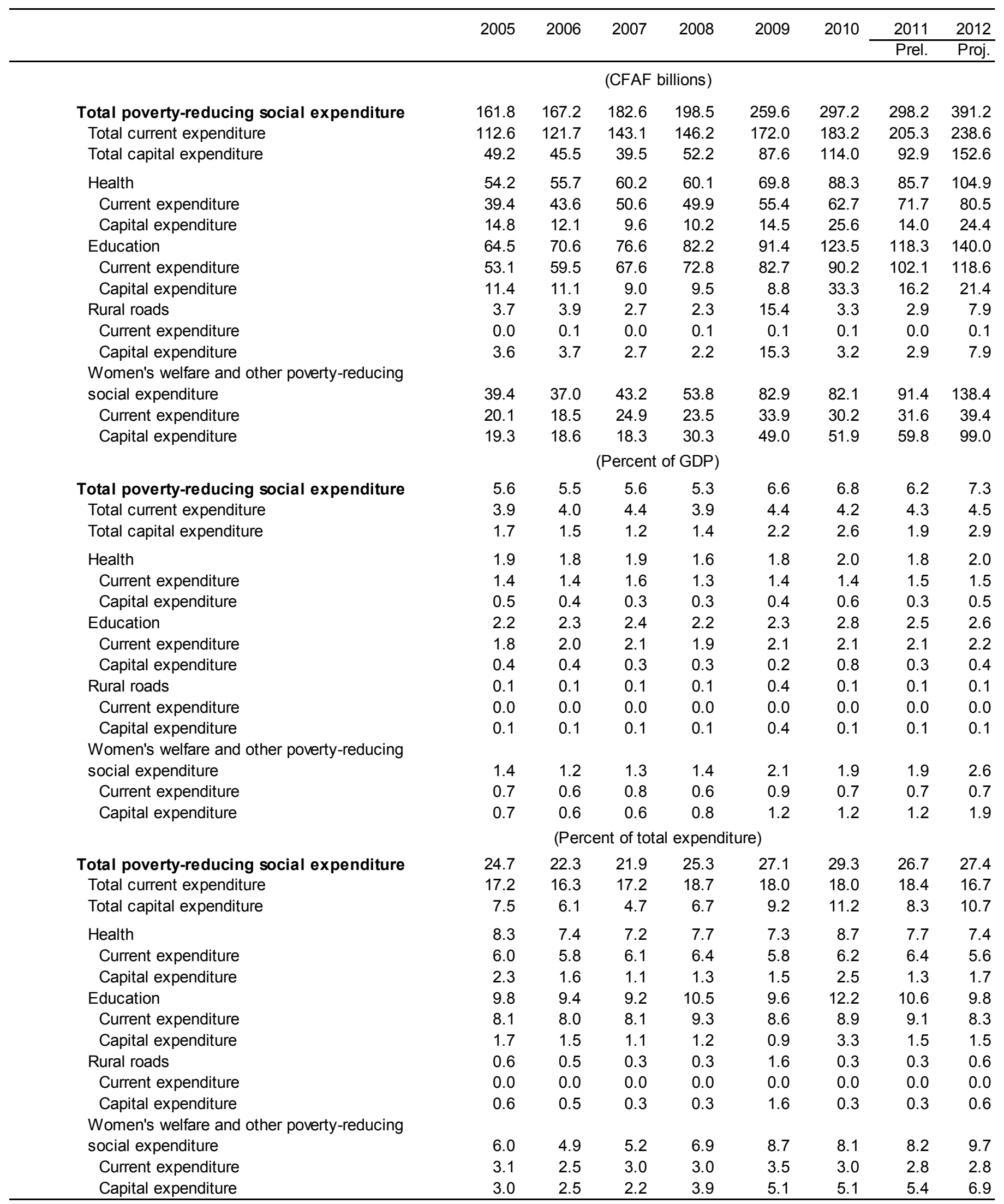

Sources: Burkinabè authorities; and IMF staff estimates and projections. 
Table 9. Burkina Faso: Indicators of Capacity to Repay the IMF, 2010-19

\begin{tabular}{|c|c|c|c|c|c|c|c|c|c|c|}
\hline & 2010 & 2011 & 2012 & 2013 & 2014 & 2015 & 2016 & 2017 & 2018 & 2019 \\
\hline & & \multicolumn{9}{|c|}{ Projections } \\
\hline IMF obligations based on existing credit & \multicolumn{10}{|c|}{ (SDR millions) } \\
\hline Principal & 0.0 & 2.4 & 4.7 & 5.1 & 7.2 & 14.1 & 14.5 & 14.7 & 14.3 & 12.3 \\
\hline Charges and interest & 0.0 & 0.1 & 0.0 & 0.0 & 0.2 & 0.2 & 0.2 & 0.1 & 0.1 & 0.1 \\
\hline IMF obligations based on existing and prospective credit & \multicolumn{10}{|c|}{ (SDR millions) } \\
\hline Principal & 0.0 & 2.4 & 4.7 & 5.1 & 7.2 & 14.1 & 14.5 & 17.8 & 24.8 & 23.4 \\
\hline Charges and interest & 0.0 & 0.1 & 0.0 & 0.0 & 0.4 & 0.3 & 0.3 & 0.3 & 0.2 & 0.2 \\
\hline \multicolumn{11}{|l|}{ Total obligations based on existing and prospective credit } \\
\hline SDR millions & 0.0 & 2.5 & 4.7 & 5.1 & 7.5 & 14.5 & 14.8 & 18.1 & 25.0 & 23.5 \\
\hline CFAF billions & 0.0 & 1.8 & 3.6 & 3.9 & 5.8 & 11.1 & 11.4 & 13.9 & 19.2 & 18.1 \\
\hline Percent of exports of goods and services & 0.0 & 0.1 & 0.3 & 0.2 & 0.3 & 0.5 & 0.5 & 0.6 & 0.8 & 0.7 \\
\hline Percent of debt service ${ }^{7}$ & 0.0 & 5.8 & 9.0 & 9.5 & 12.8 & 21.2 & 19.9 & 23.5 & 27.7 & 24.6 \\
\hline Percent of GDP & 0.0 & 0.0 & 0.1 & 0.1 & 0.1 & 0.2 & 0.2 & 0.2 & 0.2 & 0.2 \\
\hline Percent of tax revenue & 0.0 & 0.3 & 0.5 & 0.5 & 0.6 & 1.1 & 1.0 & 1.1 & 1.3 & 1.2 \\
\hline Percent of quota & 0.0 & 4.1 & 7.8 & 8.5 & 12.5 & 24.0 & 24.5 & 30.0 & 41.5 & 39.1 \\
\hline \multicolumn{11}{|l|}{ Outstanding IMF credit } \\
\hline SDR millions & 83.6 & 94.1 & 138.4 & 139.8 & 132.6 & 118.5 & 104.0 & 86.2 & 61.5 & 38.1 \\
\hline CFAF billions & 63.1 & 70.0 & 106.5 & 107.6 & 102.1 & 91.2 & 80.1 & 66.4 & 47.3 & 29.3 \\
\hline Percent of exports of goods and services & 6.7 & 5.7 & 7.4 & 6.5 & 5.4 & 4.5 & 3.6 & 2.8 & 1.8 & 1.1 \\
\hline Percent of debt service $^{1}$ & 252.8 & 220.4 & 263.7 & 260.2 & 227.2 & 173.6 & 140.2 & 112.0 & 68.2 & 39.8 \\
\hline Percent of GDP & 1.4 & 1.5 & 2.0 & 1.9 & 1.6 & 1.3 & 1.1 & 0.8 & 0.5 & 0.3 \\
\hline Percent of tax revenue & 11.1 & 10.1 & 14.3 & 13.0 & 10.8 & 8.7 & 6.7 & 5.1 & 3.3 & 1.9 \\
\hline Percent of quota & 138.9 & 156.3 & 229.9 & 232.2 & 220.3 & 196.8 & 172.8 & 143.3 & 102.1 & 63.2 \\
\hline Net use of IMF credit (SDR millions) & 13.9 & 10.5 & 44.3 & 1.4 & -7.2 & -14.1 & -14.5 & -17.8 & -24.8 & -23.4 \\
\hline Disbursements & 13.9 & 12.9 & 49.0 & 6.5 & 0.0 & 0.0 & 0.0 & 0.0 & 0.0 & 0.0 \\
\hline Repayments and Repurchases & 0.0 & 2.4 & 4.7 & 5.1 & 7.2 & 14.1 & 14.5 & 17.8 & 24.8 & 23.4 \\
\hline \multicolumn{11}{|l|}{ Memorandum items: } \\
\hline Exports of goods and services (CFAF billions) & 935.7 & 1236.2 & 1445.9 & 1650.0 & 1882.7 & 2048.1 & 2212.5 & 2371.9 & 2560.7 & 2765.9 \\
\hline External debt service (CFAF billions) ${ }^{1}$ & 24.9 & 31.8 & 40.4 & 41.3 & 44.9 & 52.5 & 57.1 & 59.2 & 69.4 & 73.7 \\
\hline Nominal GDP (CFAF billions) & 4,371 & 4,807 & 5,326 & 5,813 & 6,345 & 6,925 & 7,550 & 8,225 & 8,952 & 9,733 \\
\hline Tax revenue (CFAF billions) & 565.7 & 695.6 & 745.3 & 827.9 & 941.8 & 1048.9 & 1194.5 & 1310.3 & 1431.4 & 1562.2 \\
\hline Quota (SDR millions) & 60.2 & 60.2 & 60.2 & 60.2 & 60.2 & 60.2 & 60.2 & 60.2 & 60.2 & 60.2 \\
\hline
\end{tabular}

${ }^{1}$ Total external debt service includes IMF repurchases and repayments. 
Table 10. Burkina Faso: Schedule of Disbursements Under the ECF Arrangement, 2010-13

\begin{tabular}{|c|c|c|}
\hline Amount & Date Available & Conditions Necessary for Disbursement ${ }^{1}$ \\
\hline SDR 7.454 million & June 14, 2010 & $\begin{array}{l}\text { Following Executive Board approval of the new } \\
\text { ECF arrangement }\end{array}$ \\
\hline SDR 6.45 million & December 15, 2010 & $\begin{array}{l}\text { Observance of the performance criteria for June } \\
30,2010 \text {, and completion of the first review under } \\
\text { the arrangement }\end{array}$ \\
\hline SDR 6.45 million & June 14, 2011 & $\begin{array}{l}\text { Observance of the performance criteria for } \\
\text { December } 31,2010 \text {, and completion of the } \\
\text { second review under the arrangement }\end{array}$ \\
\hline SDR 6.45 million & December 15, 2011 & $\begin{array}{l}\text { Observance of the performance criteria for June } \\
30,2011 \text {, and completion of the third review } \\
\text { under the arrangement }\end{array}$ \\
\hline SDR 30.53 million & June 8, 2012 & $\begin{array}{l}\text { Observance of the performance criteria for } \\
\text { December } 31,2011 \text {, and completion of the fourth } \\
\text { review under the arrangement }\end{array}$ \\
\hline SDR 18.49 million & December 1, 2012 & $\begin{array}{l}\text { Observance of the performance criteria for June } \\
30,2012 \text {, and completion of the fifth review under } \\
\text { the arrangement }\end{array}$ \\
\hline SDR 6.45 million & May 1, 2013 & $\begin{array}{l}\text { Observance of the performance criteria for } \\
\text { December } 31,2012 \text {, and completion of the sixth } \\
\text { review under the arrangement }\end{array}$ \\
\hline
\end{tabular}

${ }^{1}$ In addition to the generally applicable conditions under the Extended Credit Facility. 
Table 11. Burkina Faso: Financial Soundness Indicators, 2007-11

\begin{tabular}{|c|c|c|c|c|c|}
\hline & 2007 & 2008 & 2009 & 2010 & 2011 \\
\hline & \multicolumn{5}{|c|}{ (in percent, unless otherwise mentionned) } \\
\hline \multicolumn{6}{|l|}{ Capital adequacy } \\
\hline Capital to total assets & 8.4 & 8.3 & 7.4 & 6.6 & 5.9 \\
\hline Regulatory capital to risk-weighted assets & 13.0 & 12.4 & 12.4 & 6.4 & n.a \\
\hline Non performing loans to capital & 47.0 & 34.1 & 32.8 & 75.3 & 49.0 \\
\hline \multicolumn{6}{|l|}{ Asset composition and quality } \\
\hline Total loans to total assets & 60.4 & 59.2 & 54.5 & 52.5 & 55.0 \\
\hline Gross Non Performing Loans to total loans & 17.5 & 15.8 & 5.8 & 9.5 & 5.2 \\
\hline Provisions to Non Performing Loans & 66.3 & 66.7 & 334.6 & 144.6 & 203.7 \\
\hline NPLs net of provisions to total loans & 6.7 & 5.9 & 5.8 & -4.2 & -5.4 \\
\hline \multicolumn{6}{|l|}{ Earnings and profitability } \\
\hline After-tax return on average assets & 0.6 & -0.5 & 1.1 & n.a & n.a \\
\hline After-tax return on average equity & 6.7 & -4.7 & 11.4 & n.a & n.a \\
\hline Interest margin to gross income & 37.0 & 46.7 & n.a & n.a & n.a \\
\hline Noninterest expenses to gross income & 76.0 & 86.1 & 70.4 & 129.4 & n.a \\
\hline \multicolumn{6}{|l|}{ Liquidity } \\
\hline Liquid assets to total assets & 43.7 & 42.4 & n.a & n.a. & n.a. \\
\hline Liquid assets to short term liabilities & 71.6 & 67.5 & n.a & n.a. & n.a. \\
\hline Total deposits/Total loans & 96.2 & 93.6 & 106.7 & 59.8 & 0.0 \\
\hline \multicolumn{6}{|c|}{ Compliance levels (number of compliant banks) ${ }^{1 /}$} \\
\hline Minimum statutory capital & 7 & 9 & 6 & 10 & 10 \\
\hline Capital adequacy ratio (CAR) & 9 & 11 & 8 & 10 & 10 \\
\hline Liquidity ratio & 7 & 7 & 7 & 10 & 10 \\
\hline Portfolio structure ratio & 0 & 0 & 0 & 0 & 0 \\
\hline
\end{tabular}

Source: BCEAO and staff estimates.

1 As of Dec 2011, Burkina Faso had 11 banks. 


\section{APPENDIX I. LETTER OF INTENT}

Ouagadougou, May 24, 2012

Madame Christine Lagarde

Managing Director

International Monetary Fund

700 19th St., N.W.

Washington, D.C.

Madame Managing Director:

The government of Burkina Faso has continued to implement the measures foreseen in its economic program supported by the IMF Extended Credit Facility (ECF) arrangement.

The program implementation took place in an environment marked by social unrest during the first half of 2011, low rainfall, and the international economic and financial crisis, leading to a marked deceleration in growth. Despite the difficult context, the government implemented its economic and financial program satisfactorily, in keeping with its commitments under the December 7, 2011 Memorandum of Economic and Financial Policies (MEFP). The attached MEFP supplements these documents.

All of the quantitative performance criteria for the completion of the fourth review of the ECF program were met. Most of the structural reforms agreed for end-December 2011 were implemented, and all quantitative criteria were achieved with the exception of the indicative target for social expenditures, which represented 94 percent of the target level. In light of these results and based on the policies set out in the attached MEFP, we request that the IMF conclude the fourth review of the program under the ECF arrangement, and issue the fifth disbursement totaling SDR 6.45 million.

In 2012, the government intends to vigorously pursue implementation of the Accelerated Growth and Sustainable Development Strategy (SCADD) to strengthen the economy's resilience to various shocks while promoting inclusive growth and poverty reduction. However, the food crisis brought on by the lack of rainfall, the assistance to refugees fleeing conflicts in the subregion - currently estimated at about 57,000 individuals with over 120,000 head of cattle - and increased international oil prices have considerably weakened our financial position.

The government of Burkina Faso is determined to overcome these challenges in close cooperation with its technical and financial partners, as set out in the attached MEFP. In 
this regard, the government intends to pursue the implementation of reforms supported by the ECF, particularly the reforms to mobilize domestic revenue and strengthen public financial management, while implementing the priority public investments identified in the SCADD.

In order to facilitate the adjustment and address significant additional financing needs without jeopardizing the objectives of the SCADD, the government requests increased access under the ECF arrangement in an amount equivalent to 60 percent of its quota share, or SDR 36.12 million, and that SDR 24.08 million of this amount be disbursed upon the completion of the fourth review.

The government believes that the measures presented in the MEFP will serve to achieve the economic and social objectives of its program. It is determined nonetheless to take any further measures that may prove necessary to this end. The government will consult with the IMF before adopting such measures, and in any event before modifying the measures provided in the MEFP, in accordance with applicable IMF policies. Furthermore, the government will provide the IMF with information on implementation of the agreed measures and program execution, as provided in the attached revised Technical Memorandum of Understanding or at the request of the IMF.

As in the past, the government agrees to the publication of this letter, the attachments hereto, and the related IMF staff report upon approval by the IMF Executive Board.

Very truly yours,

$$
\text { /s/ }
$$

\section{Lucien Marie Noël Bembamba}

Officier de l'Ordre National

Attachments: 2012-2013 Memorandum of Economic and Financial Policies Revised Technical Memorandum of Understanding 


\section{Attachment I. Memorandum of Economic and Financial Policies for 2012-2013}

\section{INTRODUCTION}

1. This memorandum updates Burkina Faso's economic and financial program in the context of the three-year arrangement concluded with the IMF under the Extended Credit Facility (ECF). It presents recent economic developments, the results achieved during the second half of 2011, and the outlook for the period 2012-2013. The arrangement, which was approved by the IMF Executive Board on June 14, 2010, aims to consolidate macroeconomic stability, improve prospects for growth, and intensify efforts to reduce poverty. This memorandum updates and supplements the memorandum of December 7, 2011.

\section{Recent Economic Developments And Results of Program ImPlementation AT END-DECEMBER 2011}

\section{A. Recent Economic Developments}

2. In a subregional and national context of inadequate rainfall, the growth of the Burkinabè economy slowed in 2011. According to estimates, real GDP growth was 4.2 percent compared to 7.9 percent in 2010 , reflecting weak grain production which was not offset in full by the recovery of cotton production and strong mining production:

- Gold production increased from 23.07 tons in 2010 to 32.6 tons in 2011, or an increase of over 41 percent.

- In the agriculture sector, grain production, estimated at 3,666,405 tons, declined by 19.6 percent from the previous season due to unfavorable climate conditions that diminished the impact of the government's actions in the past three years to improve the productivity of the sector. These included: (i) providing agricultural producers with equipment and improved seeds; (ii) increasing the use of organic fertilizers; (iii) training, organization, and advisory services for farm households.

- The production of seed cotton recovered in 2011, increasing to 414,500 tons from 335,398 tons in 2010 . The increase is attributable to the increase in planted areas and an improvement in yields in the order of 6 percent over the previous crop year.

- Tertiary sector activities, which expanded by 5.2 percent, benefited from official events organized in 2010 such as the Ouagadougou International Tourism and Hotel Trade Show (SITHO) and the Ouagadougou Pan African Film Festival (FESPACO).

3. Consumer prices increased overall relative to 2010, although inflation remained below the WAEMU standard of 3 percent. The annual average inflation rate was 2.7 percent compared to -0.6 percent the previous year, essentially reflecting the sharp rise in the price of cereal products at year end. 
4. The current account improved in 2011. The effect of strong international gold and cotton prices mitigated the impact of the continued rise in international oil prices. The current account balance in 2011 improved by 1.1 percent of GDP compared to 2010 .

5. The money supply increased by $\mathbf{1 2 . 8}$ percent compared to end-December $\mathbf{2 0 1 0}$, reflecting increased net foreign assets and credit to the private sector. With the buoyancy of the secondary and tertiary sectors, credit to the economy increased by 23.5 percent compared to end-December 2010.

6. The financial sector remains sound. The net rate of bank portfolio deterioration improved moderately, from 3.72 percent in 2010 to 2.96 percent in 2011. The government, through the Burkinabè Economic and Social Development Fund (FBDES), supported the effort of certain financial institutions in the transition to compliance with the West African Monetary Union (WAMU) decision on increasing banks' minimum capital.

\section{The fiscal position further improved in $\mathbf{2 0 1 1}$ following strong revenue} mobilization and improved control of spending despite the social unrest during the first half of the year. With regard to revenue, measures to increase the accountability of collection units and monitor those units more closely generated 11.5 percent more revenue than planned. Total revenue increased by 0.9 percentage points of GDP, from 15.6 percent in 2010 to 16.5 percent of GDP in 2011. Tax revenue increased to 14.5 percent of GDP. This performance includes the one-off collection of corporate tax with no deduction of prepayments, as 2011 was the first year the tax went into effect. Without this factor, the tax burden represented approximately 13.8 percent of GDP. The rising price of gold also boosted revenue. With regard to expenditure, execution remained relatively low, given delays in executing contracts following the social unrest of the first half of 2011. Total expenditure and net lending came to 24.3 percent of GDP compared to 24.6 percent of GDP in 2010.

\section{The overall budget deficit (cash basis, including grants) came to 2.2 percent of} GDP in 2011 compared to 4.6 percent in 2010. The deficit was financed primarily by external resources in the amount of CFAF 129.1 billion (2.7 percent of GDP). IMF financing under the Extended Credit Facility totaled CFAF 9.7 billion. Also, a bond issue on the regional market in 2011 raised CFAF 59.1 billion, which was recorded in January 2012.

\section{B. Results of Program Implementation}

9. Program implementation was satisfactory. All the performance criteria at endDecember 2011 were achieved: (i) the total deficit, as defined in the November 17, 2010 technical memorandum of understanding, stood at CFAF 117.6 billion compared to an adjusted ceiling of CFAF 211.1 billion; (ii) the government did not contract or guarantee external debt under nonconcessional terms; (iii) the government did not contract or guarantee short-term foreign debt; and (iv) no payment arrears were accumulated on external debt. 


\section{In regard to the quantitative indicators, nearly all the objectives were achieved} or exceeded: (i) the minimum total revenue of CFAF 711.7 billion was exceeded by 11.5 percent, with performance of CFAF 793.6 billion; (ii) the large taxpayer non-filer rate of 3 percent was below the 5 percent anticipated; (iii) no payment arrears were accumulated on domestic debt; (iv) social expenditure fell short of the indicative target with performance of CFAF 298.2 billion compared to the minimum of CFAF 314.4 billion, largely attributable to a lag in mobilizing resources for the fast-track education initiative (FTI). However, the execution rate for social expenditure was over 94 percent, confirming that the government effectively secured priority poverty-reducing expenditures while undertaking actions to address the social crisis.

\section{Most of the structural benchmarks identified for the fourth review were achieved:}

- The Council of Ministers adopted the new petroleum pricing mechanism on April 4, 2012 (benchmark for end-December 2011). That decision necessitated outreach and training activities on the determination of petroleum prices and dialogue with the social partners. An ad hoc adjustment in retail prices was implemented on March 31, 2012 with an increase of CFAF 50 for each petroleum product. This adjustment level proved fully consistent with the new mechanism. These decisions served to maintain the viability of the Burkinabè National Society of Hydrocarbons (SONABHY) and the security of the country's oil supply while reducing the budget pressures caused by petroleum subsidies. SONABHY is also involved in the sustained efforts to rationalize its operating costs.

- Efforts to crack down on fraud resulted in the installation of two scanners at the Ouaga road customs offices and the Bobo train station, but the start of operations of the Bobo scanner has been temporarily delayed by technical problems.

- The implementation of the ORBUS system, subsequently named the Virtual Import/Export Operations Information System (SYLVIE), to facilitate customs operations and prevent fraud commenced with the launch of work in December 2011. The process of procuring the necessary hardware is under way, and user training to make the system operational will take place in early 2013.

- In order to expand access to financial products for a large proportion of the population, the government adopted a new microfinance development strategy and 2012-2016 action plan in January 2012 to improve access to financial services.

- In the context of the inclusive dialogue organized to resolve the 2011 social crisis and the measures agreed to appease the social movements, the government, in accordance with measures provided in the previous memorandum, paid for housing and hardship allowances to officials who had not received them since December 2011. Also, payments reflecting 2010 and 2011 promotions and the 5 percent salary increase began in January 2012. The other measures aimed at furthering the update and validation of payroll records 
were put into effect, in particular: (i) the production of a monthly list by each ministry identifying personnel who have separated or are on temporary leave, in order to determine compensation; (ii) automatic determination of salaries based on electronic records validated in the Integrated Government Personnel Salary Administration System (SIGASPE); and (iii) the elimination or adjustment of allowances during monthly audits conducted while processing payroll files.

12. In March 2012, the government launched the biometric census of civil servants, which it expects to complete in December 2012. The effort will: (i) update the individual employee files; (ii) control staffing; (iii) control the payroll file; (iv) allow each employee to monitor his or her career through a secure, interactive automated link; (v) modernize personnel management; (vi) help prevent individuals from holding multiple positions or receiving pay for services not performed. This operation will facilitate interconnection of the validated biometric file with the current account during the first quarter 2013.

\section{In the area of debt management, capacity-building activities are being conducted} to correct weaknesses noted by the March 2011 World Bank DeMPA mission. These include: (i) risk management training in March 2012 with assistance from AFRITAC West; (ii) training on the SYGADE system to improve cash flow forecasts and the management of cash balances; and (iii) compliance with the deadlines for publishing public debt information. The preparation of a medium-term debt strategy is planned following the technical assistance anticipated from the World Bank and the IMF.

\section{In regard to public finance management, a number of actions were taken to} rationalize the chain of expenditure and reduce expenditure payment times: (i) the establishment of auditing units in five ministries to verify expenditure executed towards budget allocation (structural benchmark, end-March 2012) to facilitate the authorization, financial control, and payment of expenditures in these ministries; (ii) the introduction of the capability to automatically reject irregular transactions in the expenditure cycle if the causes are attributable to suppliers; (iii), the creation of a system to verify receipt of documents among actors in the public expenditure cycle in order to strengthen accountability with regards to established processing times; and (iv) the cancellation of validation request acknowledgments and draft validations if not processed by credit administrators within a certain period of time, also to enforce the processing deadlines. .

\section{Efforts continued to prepare for the transition to program budgeting, which} seeks to facilitate more effective integration of performance-driven management with budget management and better align the budget with the priorities for the SCADD. In fact, the sector strategies or policies of ministries and institutions were broken out into programs to enable them to use program budgets, an action plan was prepared, and performance indicators set up. In the context of improving stakeholders' expertise in program budgeting tools, the ministries also received training in preparing the annual performance plan (PAP). 


\section{ECONOMIC AND Financial Policies For 2012-2013}

\section{A. Macroeconomic Framework}

16. The government intends to continue implementation of the reforms needed in the context of the SCADD to increase the pace of growth and reduce poverty. It is aware that the context for program implementation in 2012 will be difficult amid the food crisis, rising oil and grain prices, and the massive influx of refugees from the conflict in northern Mali, accompanied by their livestock.

\section{Despite exogenous shocks, a 7 percent growth rate and moderate inflation are} forecast for 2012. The increased growth will be driven essentially by the anticipated recovery of agriculture sector performance, sustained by the measures identified in the SCADD to improve production. Cotton fiber exports are expected to increase by over 32 percent as a result of continued improvement in yields and the sustained rise in the producer price, which should provide a strong incentive to plant cotton. Cereal production is expected to recover by over 12 percent in 2012 and allow inflation to be contained at levels compatible with the community standard of 3 percent. The recent buoyancy of the mining and services sectors will continue. The pace of implementing the key measures of the SCADD, if accelerated, could lead to more robust growth. The government is aware that any delay in implementing those measures would have adverse consequences for growth.

\section{B. Fiscal Policy}

18. Fiscal policy reflects several exogenous and exceptional factors. These include outlays to respond to the food crisis and humanitarian needs arising from the massive influx of refugees from the Malian conflict. The number of refugees is estimated at 56,700 and could increase many times over. The government has prepared an operational plan providing for CFAF 112.3 billion in support to populations vulnerable to the food crisis. The plan provides for four implementation phases; the first phase of mitigating the crisis was executed from January to March, 2012 at a provisional cost of CFAF 20.9 billion. The second phase of mitigation and response will be executed from April to June, 2012 for CFAF 34.5 billion. The third phase, known as "response to the crisis" will be implemented from July to September, 2012 at a cost of CFAF 48.8 million. The fourth phase, which addresses recovery and rehabilitation, will be deployed from October to December, 2012 at a cost of CFAF 8.1 billion. Having undertaken the initial humanitarian actions for the Malian refugees, the government hopes that the international community will quickly step in and provide substantial support in covering the refugee assistance plan, estimated at CFAF 13 billion. The government calls on the support of the technical and financial partners, coordinated through the United Nations system, and plans to revise the 2012 budget to cover the relevant phases of the operations described above.

19. While enacting measures to strengthen resilience to exogenous shocks, the government intends to continue efforts to strengthen fiscal performance in order to 
continue to provide substantial fiscal headroom for the public investments to support more rapid growth in the context of implementing the SCADD. In 2012, the allocation of budget resources will focus primarily on: (i) strengthening support to the rural development sector, in particular rehabilitating damaged dams and reservoirs and developing areas around dams to increase the availability of arable land with irrigation; (ii) developing economic infrastructures, including rural roads to support agriculture; (iii) promoting employment and reducing unemployment among youths and women through support for job placement after training for youths and providing women with technologies; and (iv) ensuring the security of populations and investments.

\section{Total expenditure and net lending will amount to CFAF 1,419.4 billion,}

representing 26.7 percent of GDP. Efforts to control spending through continued measures to rationalize operating expenditures will remain one of the government's key concerns. Expenses will include the cost of combined municipal and legislative elections slated for December 2012, including the introduction of biometrics in voter registration, for a total of approximately CFAF 30 billion. The government also decided to support SONABHY in restoring its financial position following steep losses resulting from the rise in petroleum prices. To this end, a transfer of CFAF 15.5 billion will be made in 2012, combined with securitization of the remaining losses of CFAF 38.3 billion over 10 years. The government also intends to continue preparations for the process of awarding contracts, and will offer preference to investment projects with growth potential that are aligned with SCADD priorities.

21. Budget revenue is expected to increase in 2012 to CFAF 858.7 billion, equivalent to 16.1 percent of GDP. Revenue will benefit from the recovery of economic activity and the effect of continued enhancement of the revenue-collecting agencies' organizational structure with the addition of collection units. For the Directorate General of Taxes (DGI), efforts will focus on (i) the tax census in six urban communes; (ii) data validation for the outstanding tax liabilities file and stepped-up efforts to clear them; (ii) continued outreach, information, and communication activities to foster a sense of civic responsibility in regard to taxes, and (iv) increased coverage of tax audits. For the Directorate General of Customs (DGD), the emphasis will be on improving the inspection mechanism for mining sector operations with the preparation of the standard protocol for installation of customs posts at mining sites.

22. The overall budget deficit (commitment basis, excluding grants) is projected at 10.5 percent of GDP. The majority of the deficit will be financed by budget support from Burkina Faso's development partners coordinated under the General Framework for Budget Support (CGAB). The overall budget deficit (cash basis, including grants) is projected at 3.8 percent of GDP, and will be financed by the disbursements anticipated from the IMF, loans for specific investment projects, the proceeds of the 2011 bond issue disbursed in 2012, and a planned bond issue on the regional market for a net total of CFAF 30 billion. In order 
to cover the full amount of costs related to exogenous shocks, additional financing of CFAF 55.7 billion will be required to cover residual needs.

23. The strategic framework and choices for the 2013 budget will be based on the priorities established in the SCADD. In view of resource constraints, the government's interventions must be prioritized in order to maximize outcomes. The priority sectors identified for 2013, in which budget resources could be optimized with greatest effect, fall into three categories: (i) the production sector, particularly agriculture, livestock farming, and fish production; (ii) the sector supporting production, in which the priorities will be transportation infrastructure, energy, and the promotion of SMIs/SMEs; and (iii) the social sectors, particularly health, education, employment, and social protection. This approach in no way implies an abandonment or neglect of actions that were to support the country's development in general. It thus represents a prioritization of those sectors in which the government will focus its action in an effort to identify better pillars and foundations for development.

24. The government's intention to achieve strong, sustained growth with a multiplier effect on revenue calls for massive investment in the productive sectors such as agriculture, livestock farming, and fish production, in which Burkina Faso has comparative advantages, not only to sustain growth but also to ensure food self-sufficiency for a population whose largest component is rural. To build a modern, diversified agricultural system that is less vulnerable to climate shocks and generates jobs, the government's interventions will address the following priorities:

- managing water resources through increased hydro-agricultural development and development of low-lying river basins with the construction of new dams meeting appropriate standards, water wells for produce farmers, and continued appropriate rehabilitation of existing dams;

- strengthening linkages between agricultural research and production through the production and distribution of improved seeds and inputs and the development of adapted, effectively operational cultivation techniques (soil and climate);

- agricultural mechanization by promoting the rational introduction of agricultural machinery in large cultivation spaces organized as cooperatives or collective fields (plows, cultivators, motor pumps, tractors, and post-harvest equipment);

- advice to, and support and organization of, stakeholders to enable them to make better use of technology packages and produce higher yields; and

- developing an agro-industry with greater potential by providing adapted technologies to process agricultural commodities so as to adapt to the conditions of international competition and satisfy domestic demand. 


\section{Robust growth also calls for infrastructure supporting production, with} emphasis on quality and quantity of transportation infrastructure, increased energy coverage at lower costs, and SME/SMI promotion. In 2013, then, the government's priorities will be geared toward:

- developing specialized business areas to promote enterprise development;

- supporting the creation of SME/SMI consortia to better integrate them into the production-processing-marketing chain;

- improving the business climate and supporting institutions by reducing the cost of factors (especially energy) and strengthening the institutional and legal framework, including strengthening commercial courts;

- promoting appropriate financing for SMEs/SMIs by providing banking services in closer proximity, developing mechanisms to promote customer loyalty, and securing bank deposits, with support through adapted tax measures;

- promoting new financing tools such as financial leasing or similar products to encourage the creation and growth of SMEs and microenterprises in priority sectors and areas, particularly rural areas, with the main stakeholders.

\section{A healthy, educated work force is also indispensable to enhance wealth creation} and ensure the effectiveness of development operations, which is the reason for the choice as to the health and education sectors. The issue of employment is important in view of the extent of underemployment and unemployment. In regard to education, the efforts made to implement the 10-year education development plan yielded satisfactory results with respect to supply and quality in the basic education sector. The primary school gross enrollment ratio was 80.7 percent in 2011 compared to 77.6 percent in 2010 , while the primary school completion rate rose from 52.1 percent in 2010 to 58.2 percent in 2011, a leap of six points. The rate of births attended by skilled personnel improved, indicating progress in efforts to reduce maternal mortality. Within the health districts, the rate increased from 73.5 percent in 2010 to 76.3 percent in 2011. The government launched a special job creation program for youths and women in February 2012, which is intended to generate 54,209 jobs and 10,000 occupations, and train an average of 45,100 young urban and rural graduates per year.

\section{The significance of exogenous shocks (various crises, floods, etc.) and collateral} effects call for closer consideration of aspects relating to social protections. The government will implement the national social protection policy, which was prepared through a participatory process based on an assessment of the Burkinabè social environment, which is characterized by a disturbing degree of income poverty; a young population with multiple needs in terms of health, education, and employment; and a largely illiterate population (70 percent). This situation, combined with exogenous shocks (food crisis, energy, finance, debt, floods) is not conducive to preserving the social peace needed for the development to which Burkina Faso aspires. The government, aware of this reality, implemented targeted strategies over the past decade to promote access to basic social services and jobs for disadvantaged populations. The social safety net strategies essentially involve cash transfers, transfers in kind, subsidies, exemptions, and labor-intensive schemes. 
While these efforts have yielded progress, important challenges remain. To address them, the government will focus on improving access to basic social services for the needy, in particular:

- $\quad$ support for school enrollment and health for children of disadvantaged households;

- conditional food support for disadvantaged households;

- $\quad$ support for the empowerment of disadvantaged households;

- improved access to health care for children under age five and pregnant women;

- improved coverage of medical emergencies; and

- expedited implementation of universal provision of health services in the formal and informal sectors.

28. The mining sector developed rapidly in the past three years, and the government accordingly decided to revise the mining code and implementing regulations. The revision is intended to improve the management of mining permits and the environment surrounding mining sites, and harmonize Burkinabè policy with regional mining policies to ensure that effective, transparent management of the mining sector generates substantial resources that can be leveraged to finance the ambitious programs of the SCADD. The government has requested technical assistance from its partners including the World Bank and IMF to strengthen management capacities in the sector.

29. To finance its priorities sustainably over the medium term, the government will continue its determined efforts to create the necessary fiscal headroom. To this end, it will continue improving tax administration, in particular the performance of the mediumsized enterprise and land tax divisions. It will sustain the ongoing efforts to control operating expenditures, including the implementation of measures to reduce water, electricity, and telephone consumption.

\section{Balance of payments and external debt}

30. The current account balance is expected to deteriorate in 2012 in response to high levels of imports anticipated in view of the food crisis, the influx of refugees, higher petroleum costs, and the acquisition of capital goods. Export receipts should increase by 18.4 percent, primarily reflecting the continued rise in cotton and gold prices. However, the current account deficit (excluding grants) is expected to increase to 7.9 percent of GDP. The overall balance of payments is projected at 1.0 percent of GDP, with a residual requirement of CFAF 54.4 billion.

31. The external outlook over the medium term is expected to remain stable, with growth driven by an increasingly substantial contribution from the mining sector. This sector should soon witness diversification as manganese and zinc mines come into production.

\section{The government remains committed to maintaining a prudent external debt} policy in order to ensure the sustainability of external debt. To this end, it will continue 
to seek support from Burkina Faso's development partners in order to cover the majority of its financing needs through grants. It will also ensure that loans are contracted under concessional terms, as defined in the Technical Memorandum of Understanding accompanying this memorandum. However, in light of the profile of available loan resources, the government intends to conduct discussions to finance certain profitable projects at non-concessional borrowing rates. To strengthen its mechanism for preparing its public investment program, the government created a national project preparation fund that will strengthen both quality and diligence in preparing project documents.

\section{Structural Policies and Measures}

33. The government will sustain and consolidate progress to date in implementing the measures programmed for end-December 2012, as presented in the December 7, 2011 memorandum. Also, building on the measures implemented in 2011, it will undertake other reforms in key areas expected to contribute to the achievement of the program objectives. The overall objective by 2015 is to "achieve strong, sustained, quality growth, that generates multiplier effects in terms of increasing revenue and improved living conditions for the population, while upholding the principle of sustainable development”.

34. With regard to reducing payment times, decree 2011-734/PRES/PM/MEF of October 7, 2011 creating a verification unit for government budget expenditures was implemented through the adoption of ministerial and interministerial decisions instituting verification units at the Ministry of National Education, the Ministry of Economy and Finance, the Ministry of Agriculture and Hydraulic Resources, the Ministry of Health, the Ministry of Infrastructure and Integration, and the Ministry of Public Administration, Labor, and Social Security. This measure, which was a structural benchmark for end-March 2012, was observed and will drastically reduce the rejection rate in the expenditure cycle and therefore payment times. The verification units should become fully operational during the second half of 2012 .

35. In addition, with respect to public finance, the efforts to improve the collection and management of revenue will continue. The DGI revenue management software (SINTAX), which modernized the formerly manual collection process, will be implemented in three tax directorates of Bobo-Dioulasso by end-June 2012 (new structural benchmark). Also, to optimize information sharing between the DGI and DGD, the tax information cross-checking module will be developed in the context of the project to improve SINTAX functionalities. The module will provide an effective tool to combat tax evasion and fraud by reducing the amounts certain taxpayers are tempted to conceal. In parallel, as a medium-term effort in the context of implementing its IT master plan, the DGI plans to acquire a new revenue management application (SIGTAS). A feasibility study will be conducted for this purpose by end-2012. 
36. The operational phase of the SYLVIE project, which interconnects all parties involved in customs clearance operations in the context of facilitation and preventing smuggling, should advance significantly with the acquisition of the pilot software application by end-June 2012 and hardware to conduct tests in December 2012 (structural benchmark). The SYLVIE platform, which is expected to become available in March 2013, will increase the country's competitiveness by improving the business climate in terms of cross-border trade facilitation (export and import), an area considered weak in the Doing Business assessment, and attract more foreign direct investment.

37. To further the preparation of the study on the civil service compensation system and complete it for submission to the Council of Ministers by March 2013 (structural benchmark), the joint committee responsible for directing the study is being established and will prepare a concept paper and terms of reference. The study will assess the current compensation system in Burkina Faso and make recommendations to rationalize it. Further, the government intends to undertake and complete the administrative census and biometric enrollment of public servants by the December 2012 (structural benchmark). It is expected that the verified biometric database and the integrated personnel and payroll management system (SIGASPE) will be interconnected to improve control of civil service pay.

38. The directorate of public debt is awaiting mobilization of the technical assistance needed to prepare a medium-term debt strategy by end-December 2012 (structural benchmark). The directorate will also organize a training session on the debt sustainability framework in order to begin conducting regular debt sustainability analyses.

39. In the cotton sector, an overall strategy has already been prepared to gradually reduce the government's stake in SOFITEX (structural benchmark for June 2012). However, the cotton sector is undergoing rapid expansion in view of increased international prices, and SOFITEX's financial position is therefore expected to further improve in 2012, resulting in better offers. The process of divesting the government's majority stake in SOFITEX would then continue with an update of the company's financial assessment and the recruitment of an investment bank to support the preparation of the divestment strategy (structural benchmark for March 2013).

40. In the context of implementing the financial sector strategy, the government plans to implement the action plan for the national microfinance strategy (PA/SNMF 2012-2016). The approach will consist of creating two separate structures: an entity responsible for supervision/control, and the permanent secretariat responsible for promoting and developing the microfinance sector (SPS/PMF). The steering committee will adopt an annual work plan and execute delegated project management (MOD) contracts with private and public operators prior to end-June 2012 (structural benchmark). The three MODs or execution agencies to be designated initially are the Directorate of Microfinance (the future DSC/SFD), the Professional Association of Decentralized Financial Systems (APSFD/BF), and the Maison de l'Entreprise du Burkina Faso (MEBF). 
41. The government will also continue preparing the strategy to improve the quality of financial services offered by the national postal service (SONAPOST). This structural benchmark, initially set for end-June 2012, is being postponed to end-December 2012 in light of the time required to complete the basic studies recommended to ensure that the strategy is credible and relevant.

\section{Program monitoring}

42. The authorities intend to closely monitor the implementation of their economic and financial program. In order to ensure the program's success, they intend to take any measures that prove necessary to achieve the quantitative targets and structural benchmarks agreed with IMF staff, as presented in Tables 1 and 2 of this memorandum. Program monitoring will be conducted in accordance with the revised Technical Memorandum of Understanding, which defines the quantitative performance criteria and the requirements for regular data reporting to IMF staff.

43. Throughout the program term, the Burkinabè government will refrain from

(i) introducing restrictions on payments and transfers for current transactions, or tightening such restrictions without prior consultation with the IMF; (ii) introducing or modifying multiple exchange-rate practices; (iii) concluding bilateral payment agreements inconsistent with the provisions of Article VIII of the IMF Articles of Agreement; or (iv) introducing restrictions on imports for balance of payments purposes. 
Table 1. Burkina Faso: Quantitative Performance Criteria and Indicative Targets, 2012

(CFAF billions, cumulative from beginning of year; unless otherwise indicated)

\begin{tabular}{|c|c|c|c|c|c|c|c|c|}
\hline & \multicolumn{8}{|c|}{2012} \\
\hline & \multicolumn{2}{|c|}{ Mar. $^{6}$} & \multicolumn{2}{|c|}{ Jun. } & \multicolumn{2}{|c|}{ Sep. $^{6}$} & \multicolumn{2}{|c|}{ Dec. } \\
\hline & Prog. ${ }^{7}$ & Prog. & Prog. ${ }^{7}$ & Prog. & Prog. ${ }^{7}$ & Prog. & Prog. ${ }^{7}$ & Prog. \\
\hline \multicolumn{9}{|l|}{ Quantitative Performance Criteria } \\
\hline Ceiling on net domestic financing of central government ${ }^{1}$ & $\ldots$ & 35.0 & $\ldots$ & 68.4 & $\ldots$ & 75.3 & $\ldots$ & 89.8 \\
\hline $\begin{array}{l}\text { Ceiling on the amount of new nonconcessional external } d \\
\text { guaranteed by the government } 2,3\end{array}$ & 0.0 & 0.0 & 0.0 & 0.0 & 0.0 & 0.0 & 0.0 & 0.0 \\
\hline Accumulation of external arrears ${ }^{2}$ & 0.0 & 0.0 & 0.0 & 0.0 & 0.0 & 0.0 & 0.0 & 0.0 \\
\hline \multicolumn{9}{|l|}{ Indicative targets } \\
\hline Ceiling on the overall fiscal deficit including grants ${ }^{4}$ & 51.6 & 63.0 & 91.1 & 116.0 & 122.8 & 142.7 & 154.0 & 185.2 \\
\hline Government revenue & 168.3 & 184.5 & 349.5 & 428.8 & 521.7 & 614.5 & 826.0 & 858.7 \\
\hline Poverty-reducing social expenditures ${ }^{5}$ & 84.6 & 77.5 & 176.9 & 162.0 & 280.7 & 257.0 & 384.6 & 352.1 \\
\hline Accumulation of new domestic arrears & 0.0 & 0.0 & 0.0 & 0.0 & 0.0 & 0.0 & 0.0 & 0.0 \\
\hline
\end{tabular}

Sources: Burkinabè authorities; and IMF staff estimates and projections.

${ }^{1}$ Including on-lending of prospective IMF disbursements and including a bond of CFAF 59.1 billion that was issued in 2011 and recorded in 2012.

The ceiling on net domestic financing is to be adjusted in line with the TMU definition.

2 To be observed continuously.

${ }^{3}$ Excluding Treasury bills and bonds issued in CFA francs on the regional West African Economic and Monetary Union (WAEMU) market.

This ceiling excludes supplier credit with a maturity of one year or less.

${ }^{4}$ The ceiling on the overall fiscal deficit is to be adjusted in line with the TMU definition. It is calculated on a commitment basis.

${ }^{5} 90$ percent of budget amount.

${ }^{6}$ Indicative target.

${ }^{7}$ Third ECF Review. 
Table 2. Structural Benchmarks, January 2012-March 2013

\begin{tabular}{|c|c|c|}
\hline Measure & Rationale & Completion Date \\
\hline \multicolumn{3}{|l|}{ Fiscal management } \\
\hline $\begin{array}{l}\text { Implement the Directorate General of } \\
\text { Taxes (DGI) revenue management } \\
\text { application (SINTAX) in three tax } \\
\text { directorates of Bobo-Dioulasso ( } \$ \text { 35) }\end{array}$ & Strengthening tax administration & June 2012 \\
\hline $\begin{array}{l}\text { Develop and implement the tax } \\
\text { information management and cross- } \\
\text { checking module to optimize } \\
\text { information sharing between the DGI } \\
\text { and the Directorate General of } \\
\text { Customs (DGD) ( } ₫ \text { 35) }\end{array}$ & $\begin{array}{l}\text { Strengthening tax administration } \\
\text { through limiting fraud. }\end{array}$ & December 2012 \\
\hline $\begin{array}{l}\text { Purchase the SYLVIE pilot software } \\
\text { and test it internally with } \\
\text { Ouagadougou stakeholders in } \\
\text { preparation for nationwide deployment } \\
\text { of SYLVIE ( } ₫ 36 \text { ). }\end{array}$ & $\begin{array}{l}\text { Strengthening customs } \\
\text { administration through } \\
\text { streamlining customs procedures. }\end{array}$ & December 2012 \\
\hline \multicolumn{3}{|l|}{ Civil service } \\
\hline $\begin{array}{l}\text { Submit the conclusions of the study of } \\
\text { the civil service compensation scheme } \\
\text { to the Council of Ministers ( }(37) \text {. }\end{array}$ & $\begin{array}{l}\text { To streamline compensation } \\
\text { schemes to control wage bill and } \\
\text { improve service delivery. }\end{array}$ & March 2013 \\
\hline $\begin{array}{l}\text { Conduct and complete the } \\
\text { administrative census and biometric } \\
\text { enrollment of civil servants ( } ₫ \text { 37) }\end{array}$ & $\begin{array}{l}\text { Control wage bill through } \\
\text { limiting fraud and improve } \\
\text { service delivery. }\end{array}$ & December 2012 \\
\hline \multicolumn{3}{|l|}{ Debt management } \\
\hline $\begin{array}{l}\text { Submit a medium-term debt strategy } \\
\text { to the National Public Debt Committee } \\
\text { ( }(\mathbf{3 8})\end{array}$ & $\begin{array}{l}\text { Strengthen debt management } \\
\text { capacity. }\end{array}$ & December 2012 \\
\hline
\end{tabular}




\begin{tabular}{|c|c|c|}
\hline Cotton sector & & \\
\hline $\begin{array}{l}\text { Prepare a strategy to gradually reduce } \\
\text { the government's stake in the capital } \\
\text { of SOFITEX }(\mathbf{9} 39)\end{array}$ & $\begin{array}{l}\text { Reduce market distortions and } \\
\text { public stake. }\end{array}$ & June 2012 \\
\hline $\begin{array}{l}\text { Continue the process of divesting the } \\
\text { government's holdings in SOFITEX } \\
\text { by: } \\
\text { - updating the financial position } \\
\text { assessment } \\
\text { - recruiting an investment bank to } \\
\text { support the strategy to sell the } \\
\text { government's stake ( }(\mathbb{3} 3)\end{array}$ & $\begin{array}{l}\text { Eliminate market distortions and } \\
\text { reduce public stake. }\end{array}$ & March 2013 \\
\hline Financial sector & & \\
\hline $\begin{array}{l}\text { Execute delegated project } \\
\text { management (MOD) contracts with } \\
\text { private and public operators for } \\
\text { implementation of the national } \\
\text { microfinance strategy action plan ( } \$ \\
\text { 40) }\end{array}$ & $\begin{array}{l}\text { Deepen access to financial } \\
\text { services }\end{array}$ & June 2012 \\
\hline $\begin{array}{l}\text { Prepare the strategy to improve the } \\
\text { quality of financial services offered by } \\
\text { the national postal service } \\
\text { (SONAPOST) and submit it to the } \\
\text { SONAPOST board of directors }(\mathbb{\Phi} 41)\end{array}$ & $\begin{array}{l}\text { Deepen access to financial } \\
\text { services }\end{array}$ & December 2012 \\
\hline
\end{tabular}




\title{
AtTaChment II. TeChnical Memorandum OF Understanding
}

\author{
Ouagadougou, May 2012
}

1. This technical memorandum of understanding (TMU) defines the quantitative performance criteria, indicative targets, and structural benchmarks that will serve to assess performance under the program supported by the Extended Credit Facility (ECF). It also sets deadlines for the submission to data to IMF staff.

\section{DEFinitions}

2. Government. Unless otherwise indicated, "government" means the central administration of Burkina Faso and does not include local administrations, the central bank, or any other public or government-owned entity with autonomous legal status not included in the government flow-of-funds table (TOFE).

3. Definition of debt. For the purposes of the relevant performance criteria, the definition of debt is set out in IMF Executive Board Decision No.6230-(79/140), Point 9, as amended August 31, 2009 (Decision No. 14416-(09/91), as published on the IMF website.

4. Debt guarantees. For the purposes of the relevant performance criteria, a government debt guarantee means an explicit legal obligation to service a debt in the event of nonpayment by the borrower (through payment in cash or in kind).

5. Debt concessionality. For the purposes of the relevant performance criteria, a debt is considered concessional if it includes a grant element of at least 35 percent. ${ }^{1}$ The present value (PV) of debt at the time it is contracted is calculated by discounting the borrower's future debt service payments on the debt. ${ }^{2}$ The discount rates used for this purpose are the currency-specific commercial interest reference rates (CIRRs), published by OECD. ${ }^{3}$ For debt with a maturity of at least 15 years, the ten-year-average CIRR is used to calculate the PV of debt and, hence, its grant element. For debt with a maturity of less than 15 years, the six-month average CIRR is used. The margins for differing repayment periods $(0.75$ percent for repayment periods of less than 15 years, 1 percent

\footnotetext{
${ }^{1}$ The following page of the IMF website provides a tool to calculate the grant element of a wide range of financing packages: http://www.imf.org/external/np/pdr/conc/calculator.

${ }^{2}$ The calculation of concessionality takes all aspects of the loan agreement into consideration, including maturity, grace period, repayment schedule, upfront fees, and management fees.

${ }^{3}$ For loans denominated in currencies for which the OECD does not calculate the CIRR, the grant element calculation should be based on the (average weighted) composite CIRR of the currencies in the SDR basket.
} 
for 15 to 19 years, 1.15 percent for 20 to 29 years, and 1.25 percent for 30 years or more) are added to the ten-year and six-month CIRR averages.

6. External debt. For the purposes of the relevant performance criteria, external debt is defined as debt contracted or serviced in a currency other than the CFA franc. This definition also applies to debt among WAEMU countries. The relevant performance criteria apply to the external debt of the government, public enterprises, and other public entities in which the government holds more than 50 percent of the capital, and any private debt for which the government has extended guarantees that constitute a contingent liability for the government.

7. Reporting requirements. The government will promptly inform IMF staff when new external debt is contracted or guaranteed, including the applicable terms, within a maximum of two weeks after signature of the contract.

\section{Quantitative Performance Criteria}

8. The quantitative performance criteria proposed for June and December 2012 relate to: (i) net domestic financing as defined in paragraph 9; (ii) the contracting or guarantee of nonconcessional external debt, as defined in section 1; and (iii) the nonaccumulation of payment arrears on external debt service. The amounts provided in the program for September 2012 and March 2013 are benchmarks

\section{A. Net Domestic Financing}

9. For the purposes of the relevant performance criteria, net domestic financing is defined as the sum of (i) net bank credit to the government, including net bank credit to the treasury as defined below and other government claims and debts vis-à-vis the national banking institutions (including on-lending of IMF disbursements); (ii) the stock of unredeemed government bills and bonds held outside national commercial banks; (iii) privatization receipts; and other government claims and debts vis-à-vis national nonbank institutions. Net bank credit to the treasury is the balance of the treasury's claims and debts vis-à-vis national banking institutions. Treasury claims include the cash holdings of the Burkinabè Treasury, deposits with the central bank, deposits with commercial banks, secured obligations, and government deposits in postal checking accounts (CCP). Treasury debt to the banking system includes funding from the central bank (including statutory advances, consolidated advances, IMF financing, and refinancing of secured obligations), government securities held by the central bank, funding from commercial banks (including government securities held by commercial banks), and securitized deposits of the national postal savings fund (CNE/CCP). Net bank credit to the government is calculated based on information provided by the Central Bank of West African States (BCEAO), whose figures are deemed valid for program purposes. The foregoing items are calculated based on the government budget execution report presented each month in the government financial operations table prepared by the Ministry of Economy and Finance. 


\section{Adjustment}

10. The cumulative ceiling on net domestic financing for end-June 2012 and endDecember 2012 will be adjusted upward in the amount by which external program support, excluding grants and project loans, falls short of the projected amount, up to a maximum of CFAF 53 billion. The shortfall in external program assistance (grants and loans) will be calculated in reference to the projections in Table 1 below. The ceiling will not be adjusted downward in the event the external program assistance is higher than programmed.

\begin{tabular}{|l|c|c|c|c|}
\hline \multicolumn{5}{|c|}{ Table 1: External Program Assistance (cumulative, CFAF billions) } \\
\hline & End-March 2012 & End-June 2012 & $\begin{array}{l}\text { End-September } \\
\mathbf{2 0 1 2}\end{array}$ & $\begin{array}{l}\text { End-December } \\
\mathbf{2 0 1 2}\end{array}$ \\
\hline $\begin{array}{l}\text { Grants and } \\
\text { loans }\end{array}$ & 7.5 & 59.7 & 152.1 & 160.5 \\
\hline
\end{tabular}

11. The Ministry of Economy and Finance will forward data on net domestic financing to the IMF within six weeks after the end of each quarter.

\section{B. Non Accumulation of External Payment Arrears}

\section{Performance criterion}

12. External payment arrears are external payments due but unpaid. Under the program the government agrees not to accumulate external payment arrears on its debt except arrears arising from obligations being renegotiated with external creditors, including bilateral non-Paris Club creditors. Nonaccumulation of external arrears is a performance criterion, to be observed continuously.

\section{Reporting deadlines}

13. Data on outstanding balances, accumulation, and repayment of external arrears will be forwarded within six weeks after the end of each month. 


\section{Nonconcessional External Debt Contracted or Guaranteed by the Government}

\section{Performance criterion}

14. The government undertakes not to contract or guarantee any nonconcessional external debt beyond the ceiling indicated in MEFP Table 1. This performance criterion applies external debt as defined in paragraph 6 of this memorandum. It utilizes the concept of concessionality as defined in paragraph 5 of this memorandum. This performance criterion also applies to any private debt guaranteed by the government that constitutes a contingent government debt as defined in section I of this memorandum. In addition, this criterion applies to public enterprises, local governments, and other public sector entities (including public administrative, professional, scientific and technical agencies) unless excluded in MEFP Table 1. However, this performance criterion will not apply to Treasury bills and bonds issued in CFA francs on the WAEMU regional market, or to normal short term supplier credits. This commitment is a performance criterion to be observed continuously. It is measured on a cumulative basis from the IMF Executive Board's approval of the ECF, and no adjustment factor will apply.

\section{Reporting deadlines}

15. Details on any loan (terms and creditors) contracted by the government must be reported within four weeks of the end of each month. The same requirement applies to guarantees extended by the government.

\section{OTHER QUANTITATIVE INDICATIVE TARGETS}

16. The program also includes indicative targets for the overall deficit (commitment basis, grants included) as defined in paragraph 17 below; total government revenue; poverty-reducing social expenditures, and nonaccumulation of domestic payment arrears.

\section{A. Overall Deficit Including Grants}

\section{Definition}

17. For the program, the overall deficit including grants is valued on a commitment basis. It is defined as sum of the government's net foreign and domestic financing, measured from the financing side, plus a cash basis adjustment. Net foreign financing is the sum of new foreign borrowing less amortization. Government net domestic financing is defined as the sum of: (i) net bank credit to the government, including the sum of net bank credit to the Treasury as defined below and other government claims and debts visà-vis national banking institutions; (ii) the stock of unredeemed government bills and bonds held outside national commercial banks; and (iii) privatization receipts. Net bank credit to the treasury is defined as the balance of treasury claims and debts vis-à-vis national bank institutions. Treasury claims include cash holdings of the Burkinabè Treasury, central bank deposits, deposits with commercial banks, secured obligations, 
and government deposits in postal service checking accounts (CCP). Treasury debt to the banking system includes central bank funding (including statutory advances, consolidated advances, IMF financing, and refinancing of secured obligations), government securities held by the central bank, commercial bank funding (including government securities held by commercial banks), and securitized deposits of the national postal savings fund (CNE/CCP). Net bank credit to the government is calculated by the Central Bank of West African States (BCEAO), whose figures are deemed valid for program purposes. The stock of Treasury bills and bonds and net government external financing is calculated by the Ministry of Economy and Finance. The cash basis adjustment is defined as the sum of: (i) all unpaid expenditure commitments and (ii) the change in treasury deposits.

18. The foregoing items are calculated based on the government budget execution report presented each month in the government financial operations table prepared by the Ministry of Economy and Finance.

\section{Adjustment}

19. The overall deficit including grants will be adjusted upward in the amount by which grants fall short of the programmed amount, up to a maximum of CFAF 25 billion. It will not be adjusted if grants are higher than programmed. The shortfall will be calculated in reference to the projections in Table 2 below.

20. The overall deficit including grants will also be adjusted upward in the amount by which concessional loans exceed the programmed amount, up to a maximum of CFAF 15 billion. The surplus will be calculated in reference to the projections in Table 2 below.

\begin{tabular}{|l|c|c|c|c|}
\hline \multicolumn{5}{|c|}{ Table 2: External Budgetary Assistance (cumulative, CFAF billions) } \\
\hline & $\begin{array}{l}\text { End-March } \\
\mathbf{2 0 1 2}\end{array}$ & $\begin{array}{l}\text { End-June } \\
\mathbf{2 0 1 2}\end{array}$ & $\begin{array}{l}\text { End- } \\
\text { September } \\
\mathbf{2 0 1 2}\end{array}$ & $\begin{array}{l}\text { End-December } \\
\mathbf{2 0 1 2}\end{array}$ \\
\hline $\begin{array}{l}\text { Grants } \\
\text { (program } \\
\text { and project) }\end{array}$ & 47.9 & 154.0 & 300.2 & 376.0 \\
\hline Loans & 33.2 & 58.0 & 82.9 & 116.1 \\
\hline
\end{tabular}

\section{Reporting deadlines}

21. The Ministry of Economy and Finance will forward data to the IMF on the overall deficit excluding grants within six weeks after the end of each quarter. 


\section{B. Total Government Revenue}

\section{Definition}

22. Total government revenue is valued on a cash basis. It includes all tax and nontax revenue collected by the Directorate General of Taxation, the Directorate General of Customs, the Burkinabè Treasury, and revenue collection units at ministries and institutions. It also includes revenue from treasury checks.

\section{Reporting deadlines}

23. The Ministry of Finance and Budget will forward details on total revenue to the IMF within six weeks after the end of each month.

\section{Poverty-Reducing Social Expenditures}

\section{Definition}

24. Poverty-reducing social expenditures are defined as the expenditures of sectors sponsoring the priority programs identified in the Accelerated Growth and Sustainable Development Strategy (SCADD) to accelerate the achievement of poverty reduction objectives. The expenditures cover all spending categories for the following ministries: Primary Education; Health; Social Action and National Solidarity; Promotion of Women; Civil Servants, Labor, and Social Security (includes only the expenditures related to labor and social security); Youth, Professional Training, and Employment; Agriculture and Water; Animal Resources; and Environment and Sustainable Development. They also cover rural roads and Heavily Indebted Poor Countries (HIPC) initiative (Category 5) for infrastructures, and HIPC expenditures only for the Ministries of Communication; Mines, Extractive Industries, and Energy; Justice; and the Ministry of Economy and Finance. These expenditures are monitored directly through the budget, and the indicative threshold for the program will be 90 percent of the amount established by the budget authority.

\section{Reporting deadlines}

25. The government will forward monthly data on poverty-reducing social expenditures within six weeks after the end of each month. It will also submit the poverty-reducing expenditure execution report each quarter.

\section{Non-accumulation of Domestic Payment Arrears}

\section{Definition}

26. The government will not accumulate payment arrears on domestic obligations during the program period. This is a benchmark to be observed continuously. 


\section{Reporting deadlines}

27. Data on balances, accumulation, and repayment of arrears on domestic government obligations will be reported within four weeks after the end of each month.

\section{Additional Information for Program Monitoring}

\section{A. Public Finance}

28. The government will forward the following data to IMF staff in MS Excel format:

- The monthly government financial operations table (TOFE) and the customary appendix tables, to be forwarded within six weeks after the end of each month; if data on actual investment financed by external grants and loans are not available in time, a linear estimate of execution based on the annual projections will be used;

- Complete monthly data on domestic budgetary financing (net bank credit to the government and stock of unredeemed treasury bonds and bills), to be provided within six weeks after the end of each month;

○ Quarterly data on implementation of the public investment program, including details on financing sources, to be sent within six weeks after the end of each quarter;

- Quarterly data on the stock of external debt, external debt service, external debt contracted, and external debt repayment, to be sent within six weeks after the end of each quarter;

- Monthly data in table format on the monitoring of social poverty-reducing expenditures, to be submitted at the same intervals provided above for the TOFE;

- Monthly data on petroleum product prices and taxes, including:

(i) the price structure for the month concerned;

(ii) detailed calculation of the price structure, from the f.o.b.-MED price to the retail price;

(iii) volumes purchased and made available for consumption by the petroleum distributor (SONABHY); and

(iv) a breakdown of tax revenue from petroleum products - customs duties, tax on petroleum products (TPP), and value-added tax (VAT) — and subsidies paid; to be provided within four weeks after the end of each month;

- A monthly statement of the accounts with the treasury, broken out by major category (administrative services, state enterprises, joint public-private enterprises, public administrative enterprises, international organizations, private depositors, and others), to be provided within six weeks after the end of each month. 


\section{B. Monetary Sector}

29. Within six weeks after the end of each month, the government will forward:

o the consolidate balance sheet of monetary institutions;

○ the monetary survey, within six weeks after the end of the month for provisional data, and within ten weeks after the end of each month for final data;

$\circ$ the lending and borrowing interest rates;

- the standard bank supervision indicators for banks and nonbank financial institutions, if necessary.

\section{Balance of Payments}

30. The government will transmit the following to IMF staff:

- any revision of balance of payments data (including services, private transfers, official transfers, and capital transactions), as they occur;

- Foreign trade statistics compiled by the National Statistics Institute, within three months after the end of the month concerned; and

- Preliminary annual balance of payments data, within nine months after the end of the year concerned.

\section{Real Sector}

31. The government will transmit the following to IMF staff:

○ Disaggregated monthly consumer price indices, within two weeks after the end of each month;

○ Provisional national accounts; and

$\circ \quad$ Any revision of the national accounts.

\section{E. Structural Reforms and Other Data}

32. The government will transmit the following to IMF staff:

- Any study or official report on Burkina Faso's economy, within two weeks after publication thereof; and

- Any decision, order, law, decree, ordinance, or circular having economic or financial implications, on the date published, or no later than the date of entry into force. 
INTERNATIONAL MONETARY FUND

BURKINA FASO

Fourth Review Under the Three-Year Arrangement Under the Extended Credit Facility and Request for Modification of Performance Criteria and Augmentation of Access

\section{Informational Annex}

Prepared by the African Department

Approved by Michael Atingi Ego and Thomas Dorsey

May 24, 2012

- Relations with the Fund. Describes financial and technical assistance from the Fund and provides information on the safeguards assessment and exchange rate system. Outstanding purchases and loans amounted to SDR 91.76 million (152.43 percent of quota) at end-March 2012.

○ JMAP Implementation. Describes Bank-Fund collaboration. 


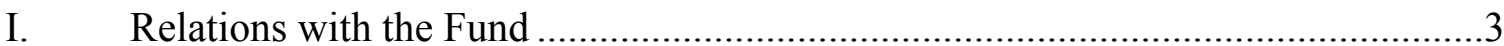

II. Joint Management Action Plan (JMAP) Implementation....................................17 


\section{Relations with the Fund}

(As at March 31, 2012)

I. Membership Status: Joined: May 2, 1963;

Article VIII

II. General Resources Account:

SDR Million

Quota

Fund holdings of currency (Exchange Rate)

Reserve Tranche Position

III. SDR Department:

Net cumulative allocation

Holdings

IV. Outstanding Purchases and Loans:

ECF Arrangements
60.20

52.72

7.50

SDR Million

57.58

48.15

SDR Million

91.76
\%Quota

100.00

87.57

12.45

\%Allocation

100.00

83.61

\%Quota

152.43

\section{Latest Financial Arrangements:}

Date of

Type

ECF

$\mathrm{ECF}^{1 /}$

$\mathrm{ECF}^{\mathbf{1 /}}$
Arrangement

Jun 14, 2010

Apr 23, 2007

Jun 11, 2003
Expiration

$\underline{\text { Date }}$

Jun 13, 2013

Apr 22, 2010

Sep 30, 2006
Amount Approved (SDR Million)

46.15

48.16

30.10
Amount Drawn

(SDR Million)

13.90

47.16

30.10

${ }^{1 /}$ Formerly PRGF.

VI. Projected Payments to Fund 1/

(SDR Million; based on existing use of resources and present holdings of SDRs):

Principal

Charges/Interest

Total
Forthcoming

\begin{tabular}{|c|c|c|c|c|}
\hline 2012 & 2013 & 2014 & 2015 & $\underline{2016}$ \\
\hline 4.7 & 5.1 & 7.2 & 14.1 & 14.5 \\
\hline$\underline{0.0}$ & $\underline{0.0}$ & $\underline{0.2}$ & $\underline{0.2}$ & $\underline{0}$ \\
\hline 4.7 & 5.1 & 7.5 & 14.5 & 14.8 \\
\hline
\end{tabular}

\footnotetext{
1/ When a member has overdue financial obligations outstanding for more than three months, the amount of such arrears will be shown in this section.
} 


\section{Implementation of HIPC Initiative:}

I. Commitment of HIPC assistance

Decision point date

Assistance committed

by all creditors (US\$ Million) ${ }^{1 /}$

Of which: IMF assistance (US\$ million)

(SDR equivalent in millions)

Completion point date

II. Disbursement of IMF assistance (SDR Million)

Assistance disbursed to the member

Interim assistance

Completion point balance

Additional disbursement of interest income ${ }^{2 /}$
Original Enhanced

Framework Framework

$\underline{\text { Total }}$

Sep 1997 Jul 2000

$229.00 \quad 324.15$

$21.70 \quad 35.88$

$16.30 \quad 27.67$

Jul 2000 Apr 2002

$\begin{array}{rrr}16.30 & 27.67 & 43.97 \\ -- & 4.15 & 4.15 \\ 16.30 & 23.52 & 39.82 \\ -- & 2.01 & 2.01 \\ 16.30 & 29.68 & 45.98\end{array}$

\footnotetext{
${ }^{1 /}$ Assistance committed under the original framework is expressed in net present value (NPV) terms at the completion point, and assistance committed under the enhanced framework is expressed in NPV terms at the decision point. Hence these two amounts cannot be added.

${ }^{2 /}$ Under the enhanced framework, an additional disbursement is made at the completion point corresponding to interest income earned on the amount committed at the decision point but not disbursed during the interim period.
}

\section{Implementation of Multilateral Debt Relief Initiative (MDRI):}

I. $\quad$ MDRI-eligible debt (SDR Million) ${ }^{1 /}$

Financed by: MDRI Trust

Remaining HIPC resources

II. Debt Relief by Facility (SDR Million)

Eligible Debt

$\begin{array}{llll}\frac{\text { Delivery }}{\underline{\text { Date }}} & \underline{\text { GRA }} & \underline{\text { PRGT }} & \underline{\text { Total }} \\ \text { January } 2006 & \text { N/A } & 62.12 & 62.12\end{array}$

${ }^{1 /}$ The MDRI provides 100 percent debt relief to eligible member countries that qualified for the assistance. Grant assistance from the MDRI Trust and HIPC resources provide debt relief to cover the full stock of debt owed to the Fund as of end-2004 that remains outstanding at the time the member qualifies for such debt relief. 


\title{
IX. Implementation of Post-Catastrophe Debt Relief (PCDR):
}

\author{
Not Applicable
}

\section{Safeguards Assessments:}

The Central Bank of West African States (BCEAO) is a common central bank of the countries of the West African Economic and Monetary Union (WAEMU). The most recent safeguards assessment of the BCEAO was completed on March 1, 2010. The 2010 update assessment found that the BCEAO continues to have controls in place at the operational level. The overall governance framework needed nonetheless to be strengthened by the addition of an audit committee to ensure that the Board of Directors exercises appropriate oversight over the control structure, including the audit mechanisms and financial statements. The Institutional Reform of the WAEMU and the BCEAO completed after the approval of the safeguards report stipulated creation of the Audit Committee, which should now start working. Efforts to implement fully the International Financial Reporting Standards reporting framework should also be pursued.

\section{Exchange Rate Arrangement:}

Burkina Faso is a member of the West African Economic and Monetary Union (WAEMU) and has no separate legal tender. The union's common currency, the CFA franc, is pegged to the euro at a rate of CFAF $655.957=$ EUR 1, consistent with the official conversions rate of the French franc to the Euro and the previous fixed rate of the CFA franc to the French franc of CFAF $100=$ F 1 . Effective January 1, 2007, the exchange arrangement of the WAEMU countries has been reclassified to the category of conventional pegged arrangement from the category of exchange arrangement with no separate legal tender. The new classification is based on the behavior of the common currency, whereas the previous classification was based on the lack of a separate legal tender. The new classification thus only reflects a definitional change, and is not based on a judgment that there has been a substantive change in the exchange regime or other policies of the currency union or its member. The exchange system is free of restrictions on the making of payments and transfers on current international transactions. 


\section{Article IV Consultations:}

The periodicity of Burkina Faso's Article IV consultation is set in accordance with the July 15, 2002 Executive Board Decision on consultation cycles. Discussions on the 2011 Article IV consultation were held between September 29-October 14, 2011 in Ouagadougou. The staff report for the 2011 Article IV consultation was considered by the Executive Board on December 21, 2011. The staff report on the previous Article IV consultation was considered by the Executive Board on December 14, 2009. The next Article IV consultation is scheduled for September 2013.

\section{ROSC/AAP:}

An FAD mission visited Ouagadougou during May 7-18, 2001 to assist the authorities undertake a draft fiscal module of a Report on the Observance of Standards and Codes (ROSC). The final report, which was issued in July 2002, found that Burkina Faso was making good progress in a number of areas to increase the transparency and accountability of government. Additional efforts are needed to bring a number of improvements to the point of implementation, particularly with regard to expenditure tracking at the local level and external audit functions. Initial discussions indicated that the authorities broadly concurred with the mission assessment. On July 31, 2002, the authorities formally adopted an action plan based on the recommendations of the final ROSC.

An STA mission during May 8-21, 2003 assisted the authorities in preparing a data ROSC. The report was published in March 2004. The mission found that most of the methodologies used in the compilation of macroeconomic statistics are in broad conformity with internationally accepted guidelines. However, most datasets are affected by limited or impaired source data arising from irregularity in the conduct of surveys (national accounts), use of outdated weights (CPI), or low response rate to surveys (balance of payments). For CPI and government finance statistics, data dissemination meets the SDDS requirement, but for other macroeconomic datasets, timeliness falls short of GDDS recommendations. The authorities broadly concurred with the main findings of the mission as well as the recommendations made to address them.

The team, jointly with World Bank staff, also discussed a HIPC Initiative Assessment and Action Plan (AAP) with the authorities. The aim was to assess the capacity of the public expenditure management system to track poverty-reducing public expenditures under the HIPC Initiative and the need for technical assistance to enhance that capacity. The mission secured the officials' approval of the jointly prepared preliminary assessment; identified the main needs for technical assistance on upgrading the capacity to track such expenditures; and drew up a draft outline action plan. This plan identifies the main needs for further technical assistance to improve tracking of poverty-reducing expenditures. The AAP has been endorsed by the authorities. 


\section{Technical Assistance:}

Significant technical assistance has been provided since 1989, more recently especially in the fiscal area:

\begin{tabular}{|c|c|c|c|}
\hline Department & $\begin{array}{l}\text { Type of } \\
\text { Assistance }\end{array}$ & Time of Delivery & Purpose \\
\hline AFRITAC & Tax Advisor & $\begin{array}{l}\text { February 7-11, } \\
2005\end{array}$ & $\begin{array}{l}\text { Review the progress made on: } \\
\text { (1) the setup of the Large Taxpayer } \\
\text { Unit and the Medium Taxpayers } \\
\text { Office; (2) the steps to strengthen } \\
\text { the fiscal control; and ( } 3 \text { ) the } \\
\text { corporate registry reform. }\end{array}$ \\
\hline AFRITAC & $\begin{array}{l}\text { Customs } \\
\text { Advisor }\end{array}$ & $\begin{array}{l}\text { February 14-18, } \\
2005\end{array}$ & $\begin{array}{l}\text { Provide assistance to control transit } \\
\text { merchandises in the country and } \\
\text { travelers at Ouagadougou airport. }\end{array}$ \\
\hline AFRITAC & $\begin{array}{l}\text { Customs, short- } \\
\text { term Expert }\end{array}$ & $\begin{array}{l}\text { February 14-23, } \\
2005\end{array}$ & $\begin{array}{l}\text { Implement customs valuation code } \\
\text { and establish a database for } \\
\text { indicative import prices. }\end{array}$ \\
\hline AFRITAC & $\begin{array}{l}\text { Microfinance } \\
\text { Advisor }\end{array}$ & March 21-25, 2005 & $\begin{array}{l}\text { Assess the TA need of the Cellule } \\
\text { responsible of the supervision of } \\
\text { microfinance institutions and } \\
\text { prepare a capacity-building } \\
\text { program. }\end{array}$ \\
\hline AFRITAC & $\begin{array}{l}\text { Short-term } \\
\text { Expert }\end{array}$ & April 18-29, 2005 & $\begin{array}{l}\text { Training of auditors of the Tax } \\
\text { administration. }\end{array}$ \\
\hline AFRITAC & PEM Advisor & $\begin{array}{l}\text { June } 27 \text {, July 1, } \\
2005\end{array}$ & $\begin{array}{l}\text { Review of the public finance } \\
\text { directives of WAEMU. }\end{array}$ \\
\hline AFRITAC & $\begin{array}{l}\text { STA Advisor } \\
\text { and Short-term } \\
\text { Expert }\end{array}$ & $\begin{array}{l}\text { June 27-July 5, } \\
2005\end{array}$ & $\begin{array}{l}\text { Assist in setting up database for the } \\
\text { TOFE (first or two scheduled } \\
\text { missions) and expand further the } \\
\text { scope of the TOFE (last of three } \\
\text { scheduled missions). }\end{array}$ \\
\hline AFRITAC & $\begin{array}{l}\text { Customs } \\
\text { Advisor visits }\end{array}$ & July $10-16,2005$ & $\begin{array}{l}\text { Advise on customs enforcement and } \\
\text { assessment of further TA needs. }\end{array}$ \\
\hline AFRITAC & Tax Advisor & August 8-12, 2005 & $\begin{array}{l}\text { (1) Review the status of } \\
\text { implementation of the } 2003 \text { FAD } \\
\text { mission's recommendations; } \\
\text { (2) update the tax directorate's }\end{array}$ \\
\hline
\end{tabular}




\begin{tabular}{|c|c|c|c|}
\hline Department & $\begin{array}{l}\text { Type of } \\
\text { Assistance }\end{array}$ & Time of Delivery & Purpose \\
\hline & & & $\begin{array}{l}\text { action plan; and (3) asses the DGI } \\
\text { TA's needs. }\end{array}$ \\
\hline AFRITAC & $\begin{array}{l}\text { STA Short-term } \\
\text { Expert }\end{array}$ & $\begin{array}{l}\text { August 22-26, } \\
2005\end{array}$ & $\begin{array}{l}\text { Assist with putting in place the } \\
\text { database of public finances. }\end{array}$ \\
\hline AFRITAC & $\begin{array}{l}\text { Customs } \\
\text { Advisor }\end{array}$ & $\begin{array}{l}\text { August } 28- \\
\text { September } 1,2005\end{array}$ & $\begin{array}{l}\text { Advise on customs enforcement and } \\
\text { assessment of further TA needs. }\end{array}$ \\
\hline AFRITAC & $\begin{array}{l}\text { STA Short-term } \\
\text { Expert }\end{array}$ & $\begin{array}{l}\text { August 29- } \\
\text { September 2, } 2005\end{array}$ & $\begin{array}{l}\text { Assist and set up database for the } \\
\text { TOFE (last of the two scheduled } \\
\text { missions). }\end{array}$ \\
\hline AFRITAC & $\begin{array}{l}\text { Microfinance } \\
\text { Advisor }\end{array}$ & $\begin{array}{l}\text { September 19-23, } \\
2005\end{array}$ & $\begin{array}{l}\text { Strengthen operational systems for } \\
\text { the surveillance of microfinance } \\
\text { institutions. }\end{array}$ \\
\hline AFRITAC & $\begin{array}{l}\text { STA Short-term } \\
\text { Expert }\end{array}$ & $\begin{array}{l}\text { September 19-23, } \\
2005\end{array}$ & $\begin{array}{l}\text { Assist and setting up database for } \\
\text { the TOFE (additional mission). }\end{array}$ \\
\hline AFRITAC & PEM Advisor & $\begin{array}{l}\text { October } 10-14, \\
2005\end{array}$ & $\begin{array}{l}\text { Review the status of } \\
\text { implementation the January } 2004 \\
\text { mission recommendations and } \\
\text { assess the technical assistance } \\
\text { needs. }\end{array}$ \\
\hline AFRITAC & $\begin{array}{l}\text { Microfinance } \\
\text { Advisor and } \\
\text { Short-term } \\
\text { Expert }\end{array}$ & $\begin{array}{l}\text { November } 21- \\
\text { December } 16,2005\end{array}$ & Coach in microfinance inspections. \\
\hline AFRITAC & STA Advisor & $\begin{array}{l}\text { December 6-15, } \\
2005\end{array}$ & $\begin{array}{l}\text { Review the WAEMU directives on } \\
\text { budgetary nomenclature. }\end{array}$ \\
\hline AFRITAC & $\begin{array}{l}\text { Customs } \\
\text { Advisor }\end{array}$ & $\begin{array}{l}\text { January } 23-25 \text {, } \\
2006\end{array}$ & $\begin{array}{l}\text { Strengthen the dialogue between the } \\
\text { customs administration and the } \\
\text { private sector (regional workshop). }\end{array}$ \\
\hline AFRITAC & STA Advisor & March 11-25, 2006 & Review the directive on the TOFE. \\
\hline
\end{tabular}


AFRITAC PEM Advisor March 13-17, 2006 Review the directive on the TOFE.

AFRITAC Tax

administration;

Short term

Expert

AFRITAC Customs

Advisor

FAD Revenue

administration

mission
March 14-28, 2006 Assist in strengthening

(1) operations of the LTU; and

(2) tax auditing.

March 14-28, 2006 Assist in defining an action plan to improve revenue mobilization.

March 14-28, 2006 Advise on tax and customs administration reform strategy (including improvement of the large tax payer unit, and strengthening of the tax and customs organization and operations).

AFRITAC Custom Advisor April 4-14, 2006

AFRITAC Tax

administration;

Short term Expert

AFRITAC Tax Advisor July 24-28, 2006

AFRITAC Microfinance Advisor

AFRITAC Real statistic September 11-20, sector Advisor 2006

AFRITAC

Microfinance, Short term

Expert

AFRITAC Public expenditure

Advisors
April 17-28, 2006

July 31-August 4, 2006

September 28-

Strengthen the control of goods valuation.

Strengthen the management of the most important taxpayers.

Follow up the implementation of the recommendations by the FAD mission in March 2006.

Identify the follow-up indicators from a distance.

Take stock of the implementation of the recommendations by the ROSC mission in March 2004.

Accompany the agents of the Cell S-IFD.

October 20, 2006

October 2-12, 2006

Review and follow up the implementation of the priority action plan in the PF strengthening strategy. 


\begin{tabular}{|c|c|c|c|}
\hline AFRITAC & $\begin{array}{l}\text { Microfinance; } \\
\text { Short term } \\
\text { Expert }\end{array}$ & October 2-20, 2006 & $\begin{array}{l}\text { Develop agent's abilities in } \\
\text { inspection and accompany them in } \\
\text { the field. }\end{array}$ \\
\hline AFRITAC & $\begin{array}{l}\text { Customs } \\
\text { administration, } \\
\text { Short-term } \\
\text { Expert }\end{array}$ & $\begin{array}{l}\text { November } 20- \\
\text { December 1, } 2006\end{array}$ & $\begin{array}{l}\text { Assess the implementation of } \\
\text { preview recommendations relating } \\
\text { to the control of goods valuation in } \\
\text { the customs. }\end{array}$ \\
\hline AFRITAC & Tax Advisor & $\begin{array}{l}\text { November } 20- \\
\text { December 1, } 2006\end{array}$ & $\begin{array}{l}\text { Strengthen capacities of the tax } \\
\text { administration to fight tax evasion. }\end{array}$ \\
\hline AFRITAC & $\begin{array}{l}\text { Macroeconomic } \\
\text { statistics } \\
\text { Advisor }\end{array}$ & March 5-9, 2007 & $\begin{array}{l}\text { Training in Government Statistics } \\
\text { Manual } 2001 .\end{array}$ \\
\hline AFRITAC & Debt Advisor & March 5-16, 2007 & $\begin{array}{l}\text { Study the situation of the } \\
\text { administrative management of the } \\
\text { internal and external debts. }\end{array}$ \\
\hline AFRITAC & $\begin{array}{l}\text { Customs } \\
\text { Advisor }\end{array}$ & March 12-23, 2007 & $\begin{array}{l}\text { Follow up the implementation of } \\
\text { the recommendations by the FAD } \\
\text { mission in March 2006, and assess } \\
\text { the implementation of the goods } \\
\text { valuation control in the customs. }\end{array}$ \\
\hline AFRITAC & $\begin{array}{l}\text { Macroeconomic } \\
\text { statistics } \\
\text { Advisor }\end{array}$ & March 19-23, 2007 & $\begin{array}{l}\text { Training in Government finance } \\
\text { statistics framework. }\end{array}$ \\
\hline AFRITAC & Tax Advisor & March 26-30, 2007 & $\begin{array}{l}\text { Assist in the implementation of the } \\
\text { tax administration strengthening } \\
\text { and modernization measures. }\end{array}$ \\
\hline AFRITAC & Tax Advisor & $\begin{array}{l}\text { May 22-June 4, } \\
2007\end{array}$ & Follow up on fiscal administration. \\
\hline AFRITAC & Custom Advisor & July 16-27, 2007 & $\begin{array}{l}\text { Computerization of customs } \\
\text { procedures. }\end{array}$ \\
\hline $\mathrm{MCM}$ & $\begin{array}{l}\text { Bank } \\
\text { supervision }\end{array}$ & July 23-27, 2007 & $\begin{array}{l}\text { Technical assistance needs } \\
\text { assessment and evaluation on bank } \\
\text { supervision. }\end{array}$ \\
\hline FAD & $\begin{array}{l}\text { Budget } \\
\text { management }\end{array}$ & $\begin{array}{l}\text { June 26-July 9, } \\
2007\end{array}$ & $\begin{array}{l}\text { Advise on tax and customs } \\
\text { administration reform strategy } \\
\text { (including improvement of the large } \\
\text { tax payer unit, and strengthening of } \\
\text { the tax and customs organization } \\
\text { and operations). }\end{array}$ \\
\hline
\end{tabular}




\begin{tabular}{|c|c|c|c|}
\hline STA & $\begin{array}{l}\text { Macroeconomic } \\
\text { statistics }\end{array}$ & $\begin{array}{l}\text { November 26- } \\
\text { December 7, } 2007\end{array}$ & $\begin{array}{l}\text { Support in setting up fiscal accounts } \\
\text { for the Treasury. }\end{array}$ \\
\hline FAD & $\begin{array}{l}\text { Tax policy } \\
\text { reform }\end{array}$ & $\begin{array}{l}\text { November } 27- \\
\text { December } 12,2007\end{array}$ & $\begin{array}{l}\text { Review and simplification of tax } \\
\text { system. }\end{array}$ \\
\hline $\begin{array}{l}\text { FAD/ } \\
\text { AFRITAC }\end{array}$ & $\begin{array}{l}\text { Public financial } \\
\text { management }\end{array}$ & & $\begin{array}{l}\text { Assist in the implementation of the } \\
\text { authorities' reform agenda in public } \\
\text { financial management. }\end{array}$ \\
\hline AFRITAC & $\begin{array}{l}\text { Debt } \\
\text { Sustainability } \\
\text { Workshop }\end{array}$ & $\begin{array}{l}\text { January } 28- \\
\text { February } 1,2008\end{array}$ & $\begin{array}{l}\text { To assist the authorities in the } \\
\text { implementation of the Debt } \\
\text { sustainability Analysis framework. }\end{array}$ \\
\hline AFRITAC & $\begin{array}{l}\text { Revenue } \\
\text { administration }\end{array}$ & $\begin{array}{l}\text { February } 11-22, \\
2008\end{array}$ & $\begin{array}{l}\text { Segmentation of taxpayers, } \\
\text { functionality of Syntax system and } \\
\text { its implementation in the medium } \\
\text { taxpayer Office. }\end{array}$ \\
\hline AFRITAC & $\begin{array}{l}\text { Micro finance } \\
\text { supervision }\end{array}$ & $\begin{array}{l}\text { February } 11-15, \\
2008\end{array}$ & $\begin{array}{l}\text { Support personnel of microfinance } \\
\text { supervision. }\end{array}$ \\
\hline STA/PEM & $\begin{array}{l}\text { UEMOA } \\
\text { Directives }\end{array}$ & $\begin{array}{l}\text { February } 25-29, \\
2008\end{array}$ & $\begin{array}{l}\text { Support the preparation of the } \\
\text { revision of UEMOA directives. }\end{array}$ \\
\hline STA & $\begin{array}{l}\text { National } \\
\text { Accounts }\end{array}$ & April 14-25, 2008 & $\begin{array}{l}\text { Train staff in national accounts } \\
\text { software and improved compilation } \\
\text { techniques and tabulation } \\
\text { procedures. }\end{array}$ \\
\hline FAD & $\begin{array}{l}\text { Tax and } \\
\text { Customs } \\
\text { Administration } \\
\text { reforms }\end{array}$ & $\begin{array}{l}\text { June 1, 2008-April } \\
2009\end{array}$ & $\begin{array}{l}\text { Advise on tax and customs } \\
\text { administration reform strategy. }\end{array}$ \\
\hline FAD & $\begin{array}{l}\text { Modernization } \\
\text { of revenue } \\
\text { administration }\end{array}$ & May 1,2008 & $\begin{array}{l}\text { Advise on the modernization of } \\
\text { revenue administration. }\end{array}$ \\
\hline STA & $\begin{array}{l}\text { Real Sector } \\
\text { Statistics } \\
\text { Advisor }\end{array}$ & $\begin{array}{l}\text { December 26, } \\
\text { 2007-December } \\
\text { 31, 2008 }\end{array}$ & $\begin{array}{l}\text { Advise on the compilation of real } \\
\text { sector statistics. }\end{array}$ \\
\hline
\end{tabular}




$\begin{array}{llll}\text { FAD } & \text { Tax Policy } & \text { March 4-13,2009 } & \begin{array}{l}\text { Review and simplification of tax } \\ \text { system }\end{array} \\ \text { FAD } & \begin{array}{l}\text { Public } \\ \text { Financial } \\ \text { Management }\end{array} & \begin{array}{l}\text { April 29-May 11, } \\ \text { Advise on elaborating a strategy to } \\ \text { operationalize program budgeting. }\end{array} \\ \text { FAD } & \text { Tax Policy } & \text { April 21-May 05, } & \begin{array}{l}\text { Review and simplification of tax } \\ \text { system. }\end{array} \\ \text { AFRITAC } & \begin{array}{l}\text { Financial } \\ \text { Supervision }\end{array} & \text { April 20-24, 2009 } & \begin{array}{l}\text { Improve the supervision of } \\ \text { microfinance institutions. }\end{array} \\ \text { MCM } & \begin{array}{l}\text { Sovereign Asset } \\ \text { and Liability } \\ \text { Management }\end{array} & \text { April 28-May 5, } & \begin{array}{l}\text { Reinforcement of the capacity of } \\ \text { the national debt committee. }\end{array} \\ \text { AFRITAC } & \begin{array}{l}\text { Public } \\ \text { Expenditure } \\ \text { Management }\end{array} & \text { April 28-May 5, } & \begin{array}{l}\text { Advise on public financial } \\ \text { management and public financial } \\ \text { statistics }\end{array} \\ & \text { 2009 } & & \end{array}$

$\begin{array}{llll}\text { STA } & \text { Statistics } & \begin{array}{l}\text { December 7-14, } \\ 2009\end{array} & \begin{array}{l}\text { Assist in extending global Financial } \\ \text { statistics coverage }\end{array} \\ \text { FAD } & \text { Tax Policy } & \begin{array}{l}\text { December 8-16, } \\ 2009\end{array} & \begin{array}{l}\text { Review and simplification of tax } \\ \text { system. } \\ \text { January 18-22, } \\ \text { FAD }\end{array} \\ \text { Technical assistance in fiscal policy }\end{array}$




\begin{tabular}{|c|c|c|c|}
\hline STA & Statistics & April 26-30, 2010 & $\begin{array}{l}\text { Assist with WAEMU directives } \\
\text { implementation }\end{array}$ \\
\hline FAD & $\begin{array}{l}\text { Public Financial } \\
\text { Management }\end{array}$ & $\begin{array}{l}\text { April 26-May 13, } \\
2010\end{array}$ & $\begin{array}{l}\text { Follow-up mission on program } \\
\text { budgeting and expenditure }\end{array}$ \\
\hline FAD & $\begin{array}{l}\text { Tax } \\
\text { administration }\end{array}$ & June $14-25,2010$ & $\begin{array}{l}\text { Customs Administration TA } \\
\text { mission }\end{array}$ \\
\hline AFRITAC & $\begin{array}{l}\text { Public Financial } \\
\text { Management }\end{array}$ & July $15-22,2010$ & $\begin{array}{l}\text { Improve PFM capacity within the } \\
\text { government }\end{array}$ \\
\hline STA & $\begin{array}{l}\text { Improve GFS } \\
\text { coverage }\end{array}$ & $\begin{array}{l}\text { July } 25-\text { August } 7 \text {, } \\
2010\end{array}$ & $\begin{array}{l}\text { Improve statistical capacity of the } \\
\text { Government }\end{array}$ \\
\hline AFRITAC & $\begin{array}{l}\text { Tax } \\
\text { administration }\end{array}$ & $\begin{array}{l}\text { July 26-August } 06 \text {, } \\
2010\end{array}$ & Increase the tax capacity of the D \\
\hline FAD & $\begin{array}{l}\text { Public Financial } \\
\text { Management }\end{array}$ & $\begin{array}{l}\text { October } 25- \\
\text { November } 5,2010\end{array}$ & $\begin{array}{l}\text { Improve the management of the } \\
\text { expenditure chain }\end{array}$ \\
\hline STA & Statistics & $\begin{array}{l}\text { December 13-17, } \\
2010\end{array}$ & Improve WAEMU statistics \\
\hline FAD & Customs & March 7-11, 2011 & Regional Workshop on customs \\
\hline FAD & $\begin{array}{l}\text { Tax } \\
\text { administration }\end{array}$ & June 1-14, 2011 & Taxation of the mining sector \\
\hline MCM & Public Debt & June 16-27, 2011 & \\
\hline FAD & Tax Policy & July 1-14, 2011 & Improve revenue mobilization \\
\hline FAD & Budgeting & July 2-22, 2011 & Program Budgeting \\
\hline FAD & $\begin{array}{l}\text { Tax } \\
\text { administration }\end{array}$ & August 1-14, 2011 & Project Management Expert \\
\hline STA & $\begin{array}{l}\text { Real Sector } \\
\text { Statistics }\end{array}$ & August 8-19, 2011 & \\
\hline
\end{tabular}




\begin{tabular}{|c|c|c|c|}
\hline STA & $\begin{array}{l}\text { Assist with } \\
\text { WAEMU GFS }\end{array}$ & $\begin{array}{l}\text { August 29- } \\
\text { September 9, } 2011\end{array}$ & \\
\hline AFRITAC & $\begin{array}{l}\text { Customs } \\
\text { Administration }\end{array}$ & $\begin{array}{l}\text { August-October } \\
2011\end{array}$ & $\begin{array}{l}\text { Define interim system for customs } \\
\text { declarations }\end{array}$ \\
\hline AFRITAC & $\begin{array}{l}\text { Tax } \\
\text { Administration }\end{array}$ & $\begin{array}{l}\text { August-October } \\
2011\end{array}$ & $\begin{array}{l}\text { Evaluation of Medium-Size } \\
\text { Enterprise Directorate }\end{array}$ \\
\hline AFRITAC & Budgeting & $\begin{array}{l}\text { August-October } \\
2011\end{array}$ & Sensitize line ministries \\
\hline AFRITAC & Budgeting & $\begin{array}{l}\text { August-October } \\
2011\end{array}$ & $\begin{array}{l}\text { Evaluation of transition toward } \\
\text { program budgeting }\end{array}$ \\
\hline AFRITAC & Budgeting & $\begin{array}{l}\text { August-October } \\
2011\end{array}$ & $\begin{array}{l}\text { Evaluation of transition toward } \\
\text { program budgeting }\end{array}$ \\
\hline AFRITAC & $\begin{array}{l}\text { Debt } \\
\text { management }\end{array}$ & $\begin{array}{l}\text { August-October } \\
2011\end{array}$ & $\begin{array}{l}\text { Support for the development of the } \\
\text { financial sector strategy }\end{array}$ \\
\hline AFRITAC & Statistics & $\begin{array}{l}\text { August-October } \\
2011\end{array}$ & Support to TOFE implementation \\
\hline AFRITAC & Statistics & $\begin{array}{l}\text { August-October } \\
2011\end{array}$ & $\begin{array}{l}\text { Support to the production of } \\
\text { quarterly accounts }\end{array}$ \\
\hline FAD & $\begin{array}{l}\text { Tax } \\
\text { Administration }\end{array}$ & Sep. 1-14, 2011 & \\
\hline FAD & Budgeting & Sep. 2-22, 2011 & $\begin{array}{l}\text { Improve quality and timeliness of } \\
\text { fiscal reporting - accounting }\end{array}$ \\
\hline FAD & $\begin{array}{l}\text { Customs } \\
\text { Administration }\end{array}$ & Sep. 5-16, 2011 & \\
\hline FAD & $\begin{array}{l}\text { Public Financial } \\
\text { Management }\end{array}$ & $\begin{array}{l}\text { September 5-16, } \\
2011\end{array}$ & \\
\hline STA & $\begin{array}{l}\text { Public Financial } \\
\text { Management }\end{array}$ & $\begin{array}{l}\text { September 5-9, } \\
2011\end{array}$ & $\begin{array}{l}\text { Improve the processing of the Table } \\
\text { of Government Fiscal Operations }\end{array}$ \\
\hline FAD & $\begin{array}{l}\text { Public Financial } \\
\text { Management }\end{array}$ & $\begin{array}{l}\text { September } 11-15 \text {, } \\
2011\end{array}$ & \\
\hline FAD & $\begin{array}{l}\text { Tax } \\
\text { administration }\end{array}$ & October 3-14, 2011 & \\
\hline FAD & $\begin{array}{l}\text { Public Financial } \\
\text { Management }\end{array}$ & $\begin{array}{l}\text { October } 10-30 \\
2011\end{array}$ & $\begin{array}{l}\text { Improve the efficiency of the } \\
\text { expenditure chain }\end{array}$ \\
\hline
\end{tabular}




\begin{tabular}{|c|c|c|c|}
\hline AFRITAC & $\begin{array}{l}\text { Debt Strategy \& } \\
\text { Management }\end{array}$ & $\begin{array}{l}\text { October 17-21, } \\
2011\end{array}$ & $\begin{array}{l}\text { Establish a debt management } \\
\text { strategy }\end{array}$ \\
\hline FAD & $\begin{array}{l}\text { Public Financial } \\
\text { Management }\end{array}$ & $\begin{array}{l}\text { November 7-18, } \\
2011\end{array}$ & \\
\hline FAD & Budgeting & $\begin{array}{l}\text { November 7-18, } \\
2011\end{array}$ & Follow-up and program budgeting \\
\hline STA & BOP & $\begin{array}{l}\text { November 14-25, } \\
2011\end{array}$ & Improve BOP and IIP \\
\hline AFRITAC & $\begin{array}{l}\text { Customs } \\
\text { Administration }\end{array}$ & $\begin{array}{l}\text { November } 2011- \\
\text { January } 2012\end{array}$ & $\begin{array}{l}\text { Follow-up and evaluation of risk } \\
\text { analysis project }\end{array}$ \\
\hline AFRITAC & Budgeting & $\begin{array}{l}\text { November 2011- } \\
\text { January } 2012\end{array}$ & $\begin{array}{l}\text { Delineation of organizations for the } \\
\text { purposes of program budgeting }\end{array}$ \\
\hline AFRITAC & $\begin{array}{l}\text { Customs } \\
\text { Administration }\end{array}$ & $\begin{array}{l}\text { November 2011- } \\
\text { January } 2012\end{array}$ & Streamlining of controls \\
\hline STA & LTE Training & $\begin{array}{l}\text { December 7-11, } \\
2011\end{array}$ & Regional Training \\
\hline
\end{tabular}

FAD Fiscal Reporting January 1-21,2012 Accounting

FAD Public Financial February 1-21, Improve the efficiency of the Management $2012 \quad$ expenditure chain

FAD Budgeting February 1-22, Follow up on progress in 2012 developing program budgeting

FAD Public Financial February 2-28, Management 2012

STA Assist with February 13-17, WAEMU PFM 2012 


$\begin{array}{llll}\text { MCM } & \begin{array}{l}\text { Public Debt } \\ \text { Management }\end{array} & \begin{array}{l}\text { February 13-24, } \\ 2012\end{array} \\ \text { AFRITAC } & \text { Budgeting } & \begin{array}{l}\text { February-April } \\ 2012\end{array} & \begin{array}{l}\text { Definition of performance } \\ \text { indicators }\end{array} \\ \text { AFRITAC } & \text { Debt } & \text { February-April } & \begin{array}{l}\text { Development of risk management } \\ \text { management }\end{array} \\ \text { AFRITAC } & \begin{array}{l}\text { Revenue } \\ \text { Administration }\end{array} & \begin{array}{l}\text { April 16-April 27, } \\ 2012\end{array} & \begin{array}{l}\text { Strengthen the management of tax } \\ \text { arrears }\end{array}\end{array}$

\section{Resident Representative:}

Ms. Isabell Adenauer took up the post of Resident Representative in June 2008. 


\section{Joint Management Action Plan (JMAP) Implementation (November 2011)}

\begin{tabular}{|c|c|c|c|}
\hline Title & Products & $\begin{array}{l}\text { Provisional } \\
\text { timing of missions }\end{array}$ & $\begin{array}{l}\text { Expected } \\
\text { delivery date }\end{array}$ \\
\hline \multicolumn{4}{|c|}{ A. Mutual information on relevant work programs } \\
\hline \multirow{8}{*}{$\begin{array}{l}\text { Bank work program } \\
\text { in next } 12 \text { months }\end{array}$} & - Public Expenditure Review (transport) & & February 2012 \\
\hline & - Mining sector study & & April 2012 \\
\hline & $\begin{array}{l}\text { - Policy Note on Growth and } \\
\text { Competitiveness }\end{array}$ & & June 2012 \\
\hline & - Poverty Assessment & ongoing & June 2012 \\
\hline & Technical Assistance & & \\
\hline & $\begin{array}{l}\text { - Support to the AICB to develop a weather } \\
\text { risk management strategy }\end{array}$ & & April-May 2012 \\
\hline & - Road map for decentralization (TA) & & $\begin{array}{l}\text { December } 2010 \text { - } \\
\text { June } 2012\end{array}$ \\
\hline & $\begin{array}{l}\text { - Global Legal Information Network } \\
\text { implementation }\end{array}$ & & May 2012 \\
\hline \multirow{6}{*}{$\begin{array}{l}\text { IMF work program } \\
\text { in next } 12 \text { months }\end{array}$} & IMF-supported program: & & \\
\hline & - Fifth ECF review and Art IV. Mission & September 2012 & December 2012 \\
\hline & Technical Assistance & & \\
\hline & - TA on Public-Private Partnerships & & \\
\hline & $\begin{array}{l}\text { - TA on Natural Resource Revenue } \\
\text { Management }\end{array}$ & & \\
\hline & - TA on Debt Management Strategy & July 2012 & August 2012 \\
\hline \multicolumn{4}{|c|}{ B. Mutual requests } \\
\hline $\begin{array}{l}\text { Fund request to } \\
\text { Bank }\end{array}$ & 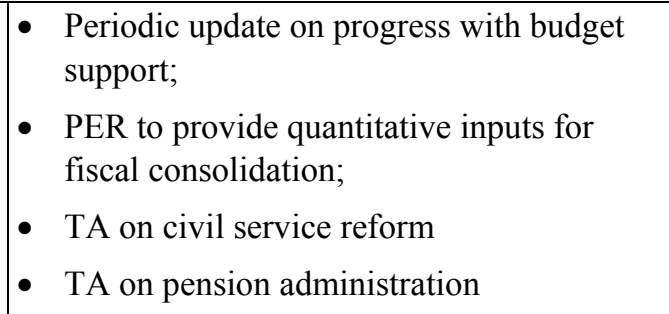 & & \\
\hline $\begin{array}{l}\text { Bank request to } \\
\text { Fund }\end{array}$ & $\begin{array}{l}\text { - Regular updates of medium-term macro } \\
\text { projections } \\
\text { - Fund Relations Note (for budget support } \\
\text { operations) }\end{array}$ & & \\
\hline \multicolumn{4}{|c|}{ C. Agreement on joint products and missions } \\
\hline $\begin{array}{l}\text { Joint products in } \\
\text { next } \\
12 \text { months }\end{array}$ & $\begin{array}{l}\text { JSAN of the progress report of the } \\
\text { SCADD }\end{array}$ & March/April 2012 & June 2012 \\
\hline
\end{tabular}




\title{
INTERNATIONAL MONETARY FUND
}

\author{
BURKINA FASO
}

\section{Debt Sustainability Analysis}

\author{
Prepared by the staffs of the International Monetary Fund \\ and the International Development Association \\ Approved by Michael Atingi-Ego and Thomas Dorsey (IMF) \\ and Jeffrey D. Lewis and Marcelo Giugale (IDA)
}

May 24, 2012

This joint World Bank/IMF DSA has been prepared in the context of authorities' request to augment access under their program supported by the IMF's Extended Credit Facility (ECF). It indicates a significant improvement in Burkina Faso's debt dynamics, based on updated gold export projections and new end-2011 debt data. ${ }^{1}$ While none of the external debt ratios under the baseline scenario or standardized stress tests breach their respective indicative debt distress thresholds, a country-specific stress test that better reflects the high dependency on projections for gold prices does result in a minor breach of the indicative debt distress threshold for the NPV of debt-to-exports. As a result, Burkina Faso's risk rating for external debt distress shifts to moderate from high.

\section{BACKGROUND AND UNDERLYING DSA ASSUMPTIONS}

1. Burkina Faso's nominal stock of debt as of end-2011 was 29.3 percent of GDP, equivalent to around US\$700 million (Table 1). Roughly 83 percent of this was external debt and the remainder was domestic debt, comprised almost entirely of 10 year government bonds.

\section{Compared to the December 2011} DSA, the main change in macroeconomic assumptions in this DSA is an increase in gold production and associated exports (Table 2). Export projections were significantly increased based gold production development in the pipeline and
Table 1. Burkina Faso: Stock of Public Debt, 2008-2011

\begin{tabular}{|c|r|r|r|r|}
\hline & $\mathbf{2 0 0 8}$ & \multicolumn{1}{c|}{$\mathbf{2 0 0 9}$} & $\mathbf{2 0 1 0}$ & \multicolumn{1}{c|}{$\mathbf{2 0 1 1}$} \\
\hline & \multicolumn{5}{|c|}{ (CFAF billions) } \\
\hline Total Debt & 883.5 & 1029.6 & 1185.2 & 1407.1 \\
\hline External & 786.4 & 867.7 & 1045.7 & 1159.1 \\
\hline Multilaterals & 604.7 & 688.5 & 853.1 & 961.0 \\
\hline Bilaterals & 181.8 & 179.2 & 192.6 & 198.1 \\
Domestic & 97.1 & 162.0 & 139.5 & 248.0 \\
\hline & & (percent of GDP) & \\
\hline Total Debt & 23.6 & 26.1 & 27.1 & 29.3 \\
\hline External & 21.0 & 22.0 & 23.9 & 24.1 \\
\hline Multilaterals & 16.2 & 17.5 & 19.5 & 20.0 \\
\hline Bilaterals & 4.9 & 4.5 & 4.4 & 4.1 \\
\hline Domestic & 2.6 & 4.1 & 3.2 & 5.2 \\
\hline
\end{tabular}

Source: Burkinabe authorities

\footnotetext{
${ }^{1}$ Based on the average CPIA score in 2008-10, Burkina Faso is ranked as a "medium performer." Burkina Faso's CPIA average for 2009-11 may move it into the category of "high performer."
} 
a slightly higher 2011 outturn than forecast (gold production was 32.4 tons vs. 31.4 tons projected in the December 2011 DSA). The December 2011 DSA had assumed that gold production would drop in 2012 and remain largely flat over the medium term. Recent information show that investments already underway in new mining capacity should bring about large increases in production over the next 2 years, and large ongoing discovery and development - over 50 additional projects are in the exploration or development phasessuggest that production should be at least 49 tons by 2015, if not much higher. Despite a marginal downward adjustment in WEO gold prices, these production volumes would lead to much larger export values.

3. Higher exports also lead to higher GDP growth and more revenues in the near term. Real GDP growth has been increased to 7.0 percent per year until 2015, and revenues are boosted by mining royalties and higher corporate income taxes. An associated reduction in the current account deficit is assumed, which is the main variable driving the accumulation of new external financing under the DS framework. Growth over the longer term, however, has been revised downward somewhat to account for the likelihood of future shocks.

4. This DSA is based on new end-2011 debt data. The authorities had revised the end2010 stock of debt upward slightly, and the outturn of the end-2011 debt stock was higher than projected (CFAF 1407 billion vs. CFAF 1246 billion projected), and higher still in GDP terms since the 2011 GDP outturn was lower than expected.

5. New external financing assumptions are somewhat more conservative. The proposed augmentation in access to the ECF-supported program (US\$55.7 million) has been included in new external borrowing in 2012. The December 2011 assumption of a gradual move from grants to loans has been maintained, but with somewhat less concessional terms for new borrowing (from an average grant element of roughly 45 percent in 2012 to about 35 percent in 2032).

Table 2. Changes in Assumptions: April 2012 DSA vs. the December 2011 DSA

\begin{tabular}{|c|c|c|c|c|c|c|c|c|}
\hline & & 2011 & 2012 & 2013 & 2014 & 2015 & 2022 & 2030 \\
\hline \multirow{2}{*}{$\begin{array}{l}\text { Gold production } \\
\text { (tons) }\end{array}$} & 2011 DSA & 31.4 & 30.6 & 32.0 & 34.7 & 34.9 & 40.1 & 48.3 \\
\hline & 2012 DSA & 32.4 & 35.0 & 40.0 & 46.1 & 49.1 & 69.1 & 86.6 \\
\hline \multirow{2}{*}{$\begin{array}{c}\text { Exports of G\& S } \\
(\% \text { of GDP) }\end{array}$} & 2011 DSA & 24.1 & 24.6 & 24.4 & 24.5 & 23.2 & 18.4 & 13.6 \\
\hline & 2012 DSA & 25.7 & 27.1 & 28.4 & 29.7 & 29.6 & 27.4 & 26.7 \\
\hline \multirow[t]{2}{*}{ GDP growth (y/y) } & 2011 DSA & 5.6 & 5.8 & 6.4 & 6.8 & 6.8 & 7.3 & 7.4 \\
\hline & 2012 DSA & 4.2 & 7.0 & 7.0 & 7.0 & 7.0 & 6.4 & 6.0 \\
\hline \multirow{2}{*}{$\begin{array}{c}\text { Revenue (\% of } \\
\text { GDP) }\end{array}$} & 2011 DSA & 15.8 & 16.2 & 16.3 & 16.6 & 17.0 & 18.5 & 19.2 \\
\hline & 2012 DSA & 16.5 & 16.1 & 16.3 & 17.0 & 17.5 & 18.5 & 19.2 \\
\hline \multirow[t]{2}{*}{ IMF (US\$ millions) } & 2011 DSA & 20.4 & 19.9 & 10.0 & 0.0 & 0.0 & 0.0 & 0.0 \\
\hline & 2012 DSA & 20.4 & 75.6 & 10.0 & 0.0 & 0.0 & 0.0 & 0.0 \\
\hline
\end{tabular}

Sources: Burkinabe authorities and staff projections. 


\title{
6. Other underlying assumptions remain the same as in the December DSA, summarized in Box 1.
}

\author{
Box 1. Burkina Faso: Macroeconomic Assumptions Underlying the DSA
}

Real GDP growth is projected at 7 percent per year until 2015, supported by projections of: (i) an increase in gold production and sustained global gold prices; (ii) improved agricultural production; and (iii) an ambitious public investment program. However, longer term real growth has been moderated to 6 percent to account for a deceleration in the rate of growth of gold production and to reflect a more conservative investment-longer term growth link, particularly in light of the frequency of weather and other shocks.

Inflation is projected to remain below 3 percent over the whole projection period. This is consistent with past performance and WAEMU macroeconomic criteria.

Current account deficit is expected to fall to 2.7 percent of GDP by 2015, in line with gold exports and somewhat higher near term imports. Over the longer term, the current account deficit is projected to increase gradually to 6 percent by 2032, as gold exports decelerate but imports remain relatively constant. The overall balance of payments remains relatively unaffected by these developments, however, since gold proceeds (after wage and supplier payments) are mainly held in offshore accounts in order to repay intra-company loans.

Fiscal deficits (including grants) are projected to decrease very gradually, from 3.3 percent of GDP in 2013 to around 2.8 percent in 2032, despite a pronounced decrease in grants (from 6.4 percent of GDP to 2 percent of GDP) and a shift toward external borrowing.

Domestic debt assumptions remain unchanged from the December 2011 DSA, that is, the nominal stock of domestic debt is held constant, resulting in a sharp decline in terms of percent of GDP. Absent a higher fiscal deficit, changing this assumption would result in a lower external financing requirement still.

\section{EXTERNAl DEbT DSA Results}

7. The December 2011 DSA maintained a determination of a "high risk" of external debt distress. This was based on a single indicator, the NPV of debt-to-exports, breaching its indicative threshold, both under the stress tests and the baseline scenario. None of the other stock variables or stress tests breached the indicative thresholds and the flow variables were far below the indicative thresholds. Indeed, the December 2011 DSA noted that it was based on conservative export assumptions and the NPV of debt-to-exports breach under the baseline scenario was 10 years later (2026) than under the 2010 DSA. The 2011 DSA concluded that further improvements in gold exports would lead to a situation where there was no breach.

\section{This DSA does not show a breach of the indicative debt distress threshold for NPV} of debt-to-exports (Tables $4 \mathrm{a}$ and $\mathrm{b}$ and Figure 2). The baseline scenario shows a slight decrease in NPV of debt-to-exports, from 53.6 percent in 2012 to 48.7 percent in 2014 (during the years of rapid growth of gold exports), followed by a steady increase to a maximum of 99.2 percent in 2032. The other debt indicators continue to show no breach in their indicative debt distress thresholds. Similarly, the standardized stress tests show no breach in the indicative thresholds.

9. Given that the DSA results are highly dependent upon gold projections, the staffs felt that consideration of a customized stress test was merited. Gold prices are inherently difficult to predict, and production projections in the outlook would also be likely to be affected 
by a significant change in prices, as this would probably affect investment. Staffs therefore ran a customized scenario based on World Bank Commodities Group projections for gold prices, which are lower than WEO projections. The effect of this change on export values was approximated by extending the standardized export shock for three further years (2013-17). World Bank baseline projections show cumulative price declines of around 40 percent over five years, with lower prices sustained over the remainder of the projection period. Even without altering production, under this scenario the debt distress threshold with respect to exports is breached.

Figure 1. PV of debt-to-exports ratio with a customized stress test

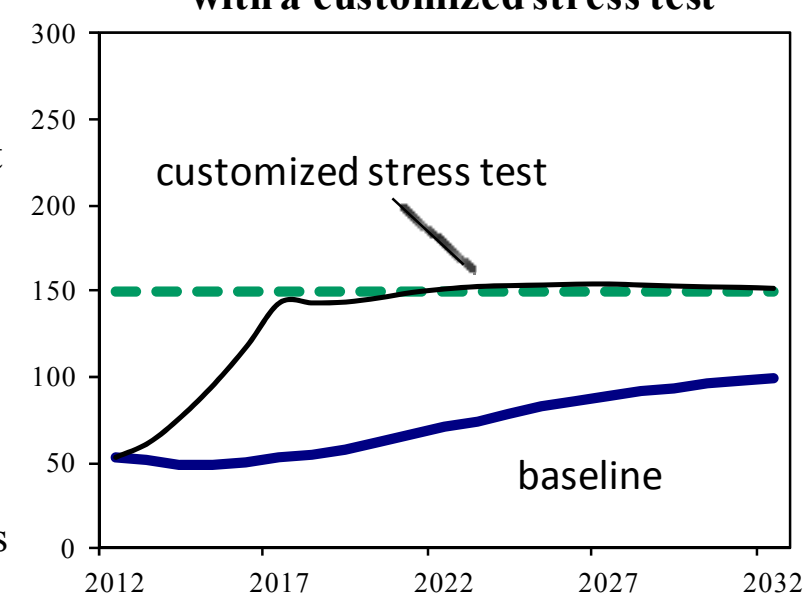
Adding any adverse impact on production would intensify this breach.

10. This DSA shows a large deviation between the historical and baseline scenarios

(Table 3). This is mainly due to a significant reduction in the current account deficit in the baseline, and thus debt accumulation, relative to the historical average. This reduction is a function of stronger export projections, and is consistent with current account performance over the last three years - those with significant gold exports - in which the current account deficit averaged just 2.5 percent of GDP. In the December 2011 analysis, it was assumed that the current account deficit returned to its pre-gold trend, hence the baseline and historical scenarios were closer. The historical scenario in this new DSA shows an improvement in debt indicators in later years, since the underlying historical averages for GDP, export, and revenue growth are significantly higher than long run projections in the baseline.

Table 3. Historical vs. Baseline: December 2011 DSA vs. April 2012 DSA

\begin{tabular}{|c|c|c|c|c|c|c|}
\hline & & GDP growth & GDP deflator & Export Growth & $\begin{array}{l}\text { CA Deficit } \\
\text { (\% of GDP) }\end{array}$ & $\begin{array}{c}\text { Revenues (\% } \\
\text { of GDP) }\end{array}$ \\
\hline \multirow{4}{*}{ Dec. 2011 DSA } & $\begin{array}{l}\text { Historical } \\
2001-2010\end{array}$ & 5.7 & 7.1 & 24.1 & 8.9 & 12.9 \\
\hline & Baseline 2011 & 5.6 & 7.2 & 50.5 & 3.3 & 15.8 \\
\hline & $\begin{array}{c}\text { Baseline } 2012 \\
17 \text { avg }\end{array}$ & 6.6 & 1.0 & 5.6 & 7.3 & 16.8 \\
\hline & $\begin{array}{c}\text { Baseline 2018- } \\
31 \text { avg }\end{array}$ & 7.3 & 1.8 & 5.8 & 6.7 & 18.6 \\
\hline \multirow{4}{*}{ April 2012 DSA } & \begin{tabular}{|c|} 
Historical \\
$2002-2011$
\end{tabular} & 5.6 & 7.9 & 29.2 & 7.8 & 13.5 \\
\hline & $\begin{array}{c}\ldots \text { of which } \\
2011\end{array}$ & 4.2 & 9.2 & 36.6 & 1.0 & 16.5 \\
\hline & $\begin{array}{c}\text { Baseline } 2012 \\
17 \text { avg }\end{array}$ & 7.0 & 1.0 & 10.1 & 3.1 & 17.3 \\
\hline & $\begin{array}{c}\text { Baseline 2018- } \\
32 \text { avg }\end{array}$ & 6.3 & 2.0 & 7.8 & 4.6 & 18.8 \\
\hline
\end{tabular}

Sources: Burkinabe authorities and IMF staff projections. 


\section{Total Public Debt DSA Results}

11. This DSA does not modify the December 2011 assumptions for the evolution of domestic debt (Box 1, Tables 5a and b, and Figure 3). Therefore, the results of the total public debt analysis mimic those of the external debt analysis, especially over the long term. However, the most extreme shock corresponds now to a shock to growth rather than the primary balance, which results in worse debt indicators under the shock than in the December DSA. The decision to leave domestic financing assumptions unchanged was taken to avoid, in the absence of a higher fiscal deficit, creating lower external financing requirements still and so that the impact of new export projections could be isolated. However, it would be reasonable to assume, in the next joint DSA, that domestic debt levels are maintained, in line with efforts to create a regional bond market and deepen financial markets.

\section{Debt Management Issues}

12. Burkina Faso has been classified at "lower debt management capacity" by the World Bank/IMF for the purposes of setting programmatic external debt limits. The authorities have enhanced debt management capacity in recent years, as noted by technical experts from the World Bank and IMF. Remaining areas for improvement include: (i) exposition of a medium-term debt management strategy (MTDS); (ii) stronger auditing procedures; (iii) better risk accounting; and (iv) an improved debt database management. The authorities have requested technical assistance from the IMF and the World Bank for the preparation of a MTDS by end-2012, and TA from the IMF to conduct a DSF workshop as a means to start preparing their own regular DSAs.

\section{Authorities VieWS}

13. The authorities concurred with the DSA results and reaffirmed their commitment to prudent borrowing policies. They noted that a move from a "high risk" rating would unlock new sources of concessional financing. They acknowledged that the rating change could result in an accelerated move away from grants toward concessional financing, and that this would require determined efforts to continue strengthening debt management capacity and increased diligence to ensure financing terms are the most generous possible.

14. The authorities stressed, however, that more flexibility is needed regarding the zero limit on nonconcessional borrowing under the ECF-supported program. They argued for consideration of some nonconcessional financing linked to high return large infrastructure projects, as yet unspecified. They would like to explore this topic in more detail at the time of the next program review.

\section{CONCLUSION}

15. Based on the results of the new DSA, Burkina Faso's risk of debt distress shifts from high to moderate. This shift primarily reflects the rapid development of Burkina Faso's gold mining sector, combined with notable improvements in underlying macroeconomic fundamentals. However, the staffs caution that any adjustments in financing plans - both on the part of the authorities and development partners - should only be undertaken gradually, to ensure that debt management capacity is sufficient to handle evolving needs. 
Table 4a. Burkina Faso: External Debt Sustainability Framework, Baseline Scenario, 2009-2032 1/

(In percent of GDP, unless otherwise indicated)

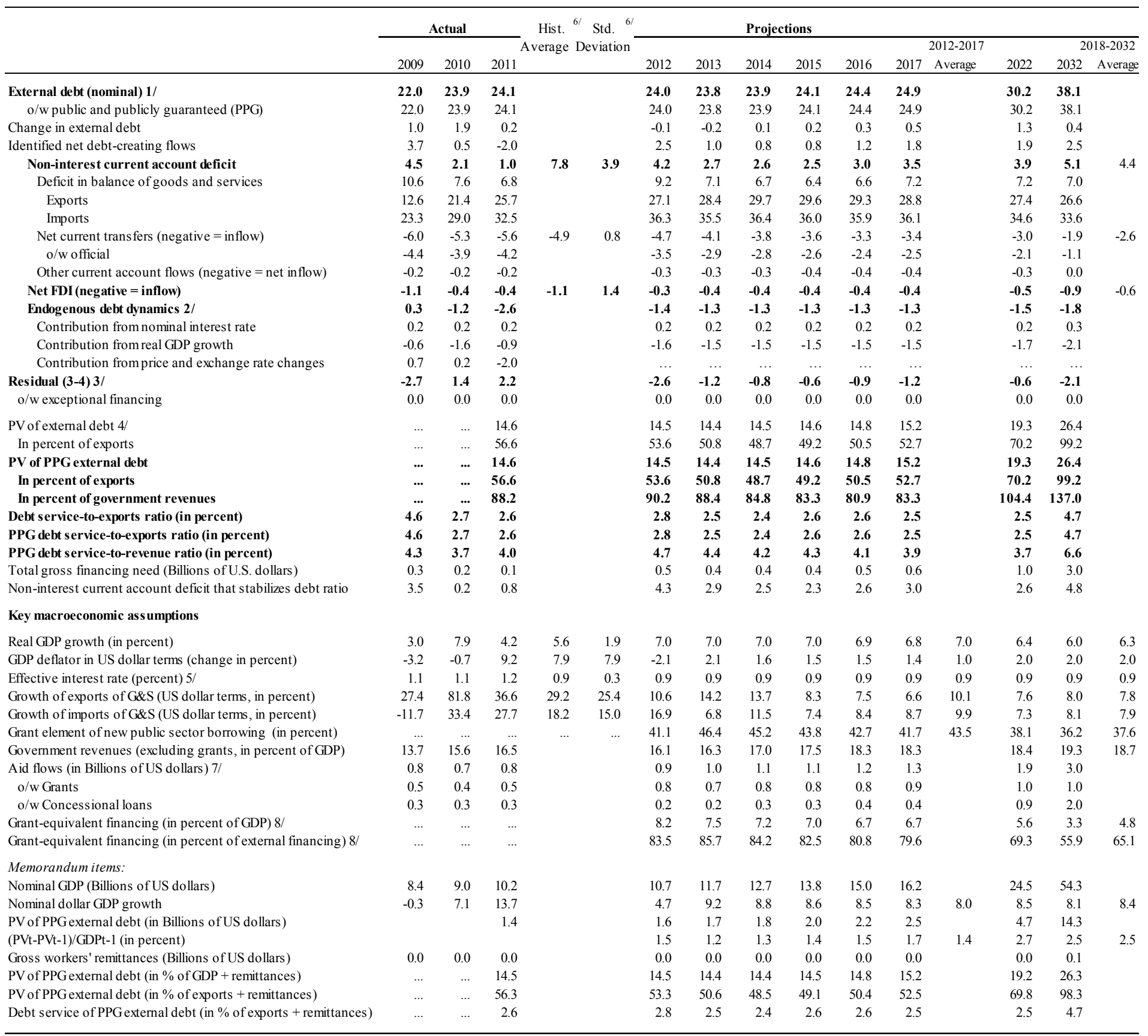

Sources: Country authorities; and staff estimates and projections.

1/ Includes both public and private sector external debt.

2/ Derived as $[\mathrm{r}-\mathrm{g}-\rho(1+\mathrm{g})] /(1+\mathrm{g}+\rho+\mathrm{g} \rho)$ times previous period debt ratio, with $\mathrm{r}=$ nominal interest rate; $\mathrm{g}=$ real GDP growth rate, and $\rho=\mathrm{growth}$ rate of GDP deflator in U.S. dollar terms.

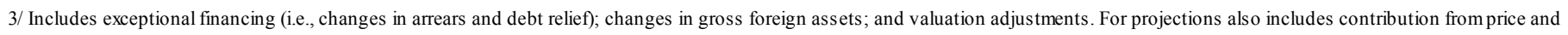
exchange rate changes.

4/ Assumes that PV of private sector debt is equivalent to its face value.

5/ Current-year interest payments divided by previous period debt stock.

6/ Historical averages and standard deviations are generally derived over the past 10 years, subject to data availability.

7/ Defined as grants, concessional loans, and debt relief.

8/ Grant-equivalent financing includes grants provided directly to the government and through new borrowing (difference between the face value and the PV of new debt). 
Table 4b. Burkina Faso: Sensitivity Analysis for Key Indicators of Public and Publicly Guaranteed External Debt, 2012-2032 (In percent)

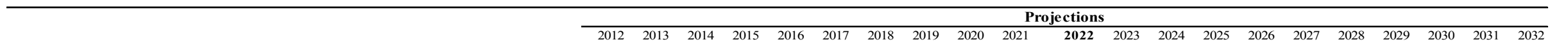

\section{PV of debt-to GDP ratio}

\section{Baseline}

\section{A. Alternative Scenarios}

A1. Key variables at their historical averages in 2012-2032 1

A2. New public sector loans on less favorable terms in 2012-2032

\section{B. Bound Tests}

B1. Real GDP growth at historical average minus one standard deviation in 2013-2014 B2. Export value growth at historical average minus one standard deviation in 2013-2014 $3 /$ B3. US dollar GDP deflator at historical average minus one standard deviation in 2013-2014

percent $20135 /$

\section{Baseline}

\section{A. Alternative Scenarios}

A1. Key variables at their historical averages in 2012-2032

A2. New public sector loans on less favorable terms in 2012-2032

\section{B. Bound Tests}

B1. Real GDP growth at historical average minus one standard deviation in 2013-2014 B2. Export value growth at historical average minus one standard deviation in 2013-2014 3/ B. US dollar GDP deflator a historical average mins one standard devation in 2013-2014 B5. Cons

B6. One-time 30 percent nominal depreciation relative to the baseline in 20135

\section{Baseline}

\section{A. Alternative Scenarios}

A1. Key variables at their historical averages in 2012-2032 1/
A2. New public sector loans on less favorable terms in 2012-2032

\section{B. Bound Tests}

B1. Real GDP growth at historical average minus one standard deviation in 2013-2014 B2. Export value growth at historical average minus one standard deviation in 2013-20143/ B4. Net non-debt creating flows at historical average minus one standard deviation in 2013-2014 4 B5. Combination of B1-B4 using one-half standard deviation shocks

B6. One-time 30 percent nominal depreciation relative to the baseline in 20135
15

$\begin{array}{lllllllllllllllllllll}15 & 17 & 19 & 21 & 22 & 24 & 25 & 26 & 27 & 29 & \mathbf{3 0} & 31 & 31 & 32 & 32 & 33 & 33 & 33 & 32 & 32 & 32 \\ 15 & 15 & 16 & 17 & 18 & 19 & 20 & 21 & 23 & 24 & \mathbf{2 6} & 28 & 30 & 31 & 33 & 34 & 35 & 36 & 37 & 38 & 39\end{array}$

$\begin{array}{lllllllllllllllllllll}15 & 15 & 15 & 15 & 16 & 16 & 17 & 17 & 18 & 19 & \mathbf{2 0} & 22 & 23 & 24 & 25 & 25 & 26 & 27 & 27 & 28 & 28 \\ 15 & 16 & 19 & 19 & 19 & 19 & 19 & 20 & 21 & 21 & \mathbf{2 2} & 23 & 23 & 24 & 25 & 25 & 26 & 26 & 27 & 27 & 27 \\ 15 & 15 & 15 & 15 & 15 & 16 & 16 & 17 & 18 & 19 & \mathbf{2 0} & 21 & 22 & 23 & 24 & 25 & 25 & 26 & 26 & 27 & 27 \\ 15 & 15 & 15 & 15 & 15 & 16 & 16 & 17 & 18 & 19 & \mathbf{2 0} & 21 & 22 & 22 & 23 & 24 & 25 & 25 & 26 & 26 & 26 \\ 15 & 14 & 13 & 13 & 13 & 14 & 14 & 15 & 16 & 17 & \mathbf{1 8} & 19 & 20 & 21 & 22 & 23 & 24 & 25 & 25 & 26 & 26 \\ 15 & 20 & 20 & 21 & 21 & 21 & 22 & 23 & 24 & 26 & \mathbf{2 7} & 29 & 30 & 31 & 33 & 34 & 35 & 35 & 36 & 37 & 37\end{array}$

\section{PV of de bt-to-exports ratio}

$\begin{array}{rrrrrrrrrrrrrrrrrrrrr}54 & 51 & 49 & 49 & 51 & 53 & 55 & 57 & 61 & 66 & \mathbf{7 0} & 75 & 79 & 83 & 86 & 89 & 92 & 94 & 96 & 98 & 99 \\ & & & & & & & & & & & & & & & & & & & & \\ 54 & 58 & 63 & 70 & 76 & 82 & 87 & 92 & 98 & 104 & \mathbf{1 0 9} & 113 & 116 & 119 & 121 & 122 & 122 & 122 & 122 & 121 & 120 \\ 54 & 53 & 53 & 56 & 60 & 65 & 69 & 74 & 81 & 88 & \mathbf{9 6} & 102 & 109 & 115 & 121 & 127 & 131 & 135 & 139 & 143 & 146 \\ & & & & & & & & & & & & & & & & & & & & \\ & & & & & & & & & & & & & & & & & & & & \\ 54 & 51 & 49 & 49 & 50 & 53 & 55 & 57 & 61 & 66 & \mathbf{7 0} & 74 & 79 & 82 & 86 & 89 & 91 & 94 & 95 & 97 & 99 \\ 54 & 62 & 77 & 77 & 78 & 80 & 82 & 84 & 88 & 92 & \mathbf{9 6} & 100 & 104 & 108 & 111 & 114 & 116 & 118 & 120 & 121 & 123 \\ 54 & 51 & 49 & 49 & 50 & 53 & 55 & 57 & 61 & 66 & \mathbf{7 0} & 74 & 79 & 82 & 86 & 89 & 91 & 94 & 95 & 97 & 99 \\ 54 & 52 & 51 & 51 & 52 & 54 & 56 & 59 & 63 & 67 & \mathbf{7 1} & 75 & 80 & 83 & 87 & 90 & 92 & 94 & 96 & 98 & 99 \\ 54 & 47 & 40 & 41 & 42 & 45 & 47 & 50 & 54 & 58 & \mathbf{6 3} & 67 & 72 & 76 & 80 & 83 & 85 & 88 & 90 & 92 & 93 \\ 54 & 51 & 49 & 49 & 50 & 53 & 55 & 57 & 61 & 66 & \mathbf{7 0} & 74 & 79 & 82 & 86 & 89 & 91 & 94 & 95 & 97 & 99\end{array}$

\section{PV of debt-to-revenue ratio}

$\begin{array}{lllllllllllllllllllll}90 & 88 & 85 & 83 & 81 & 83 & 86 & 90 & 95 & 100 & \mathbf{1 0 4} & 109 & 113 & 118 & 122 & 126 & 129 & 131 & 133 & 135 & 137\end{array}$

$\begin{array}{rrrrrrrrrrrrrrrrrrrrr}90 & 101 & 109 & 118 & 121 & 129 & 136 & 144 & 152 & 158 & \mathbf{1 6 3} & 165 & 167 & 170 & 172 & 173 & 172 & 171 & 169 & 167 & 166 \\ 90 & 93 & 93 & 95 & 96 & 102 & 109 & 117 & 125 & 134 & \mathbf{1 4 2} & 149 & 157 & 164 & 172 & 179 & 184 & 189 & 193 & 197 & 201\end{array}$

$\begin{array}{rrrrrrrrrr}90 & 91 & 90 & 88 & 86 & 88 & 92 & 96 & 101 & 106 \\ 90 & 98 & 112 & 108 & 104 & 105 & 107 & 110 & 113 & 116 \\ 90 & 90 & 88 & 86 & 84 & 86 & 89 & 94 & 98 & 103 \\ 90 & 91 & 88 & 86 & 84 & 86 & 89 & 93 & 97 & 101 \\ 90 & 85 & 74 & 73 & 72 & 74 & 78 & 82 & 87 & 93 \\ 90 & 125 & 120 & 118 & 114 & 118 & 122 & 128 & 134 & 141\end{array}$


Table 4b. Burkina Faso: Sensitivity Analysis for Key Indicators of Public and Publicly Guaranteed External Debt, 2012-2032 (continued) (In percent)

\section{Debt service-to-exports ratio}

\section{Baseline}

\section{A. Alternative Scenarios}

A1. Key variables at their historical averages in 2012-2032 1/

A2. New public sector loans on less favorable terms in 2012-2032

\section{B. Bound Tests}

B1. Real GDP growth at historical average minus one standard deviation in 2013-2014 B2. Export value growth at historical average minus one standard deviation in 2013-2014 3/ B3. US dollar GDP deflator at historical average minus one standard deviation in 2013-2014 B4. Net non-debt creating flows at historical average minus one standard deviation in 2013-2014 4/ B5. Combination of B1-B4 using one-half standard deviation shocks

B6. One-time 30 percent nominal depreciation relative to the baseline in 20135

\section{Baseline}

\section{A. Alternative Scenarios}

A1. Key variables at their historical averages in 2012-2032 1

A2. New public sector loans on less favorable terms in 2012-2032 2

\section{B. Bound Tests}

B1. Real GDP growth at historical average minus one standard deviation in 2013-2014

B2. Export value growth at historical average minus one standard deviation in 2013-2014 3/

B3. US dollar GDP deflator at historical average minus one standard deviation in 2013-2014

B4. Net non-debt creating flows at historical average minus one standard deviation in 2013-2014 4/

B5. Combination of BI-B4 using one-half standard deviation shocks

B6. One-time 30 percent nominal depreciation relative to the baseline in 20135

Memorandum item:

Grant element assumed on residual financing (i.e., financing required above baseline) 6 /

$\begin{array}{llllllllll}3 & 2 & 2 & 2 & 2 & 2 & 3 & 2 & 3 & 3 \\ 3 & 3 & 2 & 3 & 3 & 3 & 3 & 3 & 3 & 3\end{array}$

Debt service-to-revenue ratio

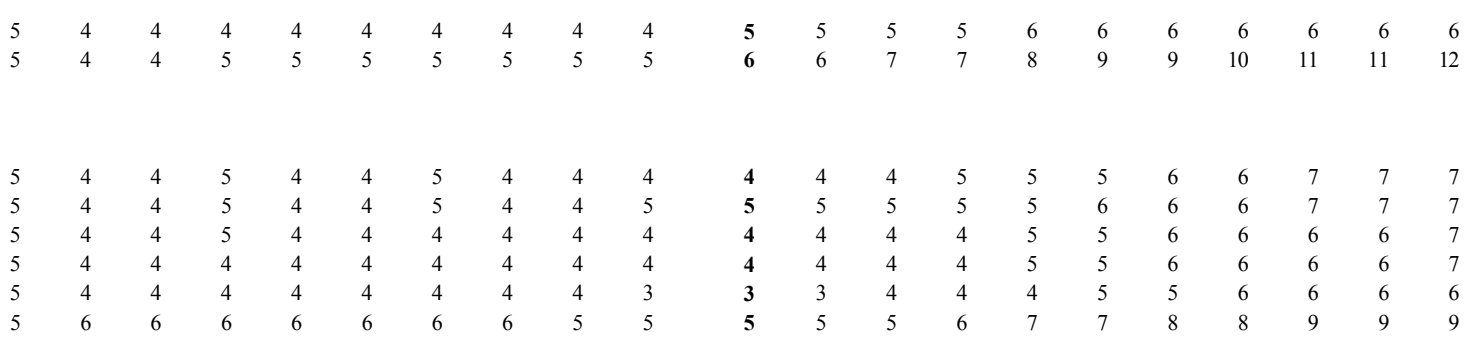

38

Sources: Country authorities; and staff estimates and projections.

1/ Variables include real GDP growth, growth of GDP deflator (in U.S. dollar terms), non-interest current account in percent of GDP, and non-debt creating flows.

2/ Assumes that the interest rate on new borrowing is by 2 percentage points higher than in the baseline., while grace and maturity periods are the same as in the baseline.

3/ Exports values are assumed to remain permanently at the lower level, but the current account as a share of GDP is assumed to return to its baseline level after the shock (implicitly assuming

an offsetting adjustment in import levels).

4/ Includes official and private transfers and FDI.

5/ Depreciation is defined as percentage decline in dollar/local currency rate, such that it never exceeds 100 percent.

6/ Applies to all stress scenarios except for A2 (less favorable financing) in which the terms on all new financing are as specified in footnote 2. 
Figure 2. Burkina Faso: Indicators of Public and Publicly Guaranteed External Debt under Alternatives Scenarios, 2012-2032 1/
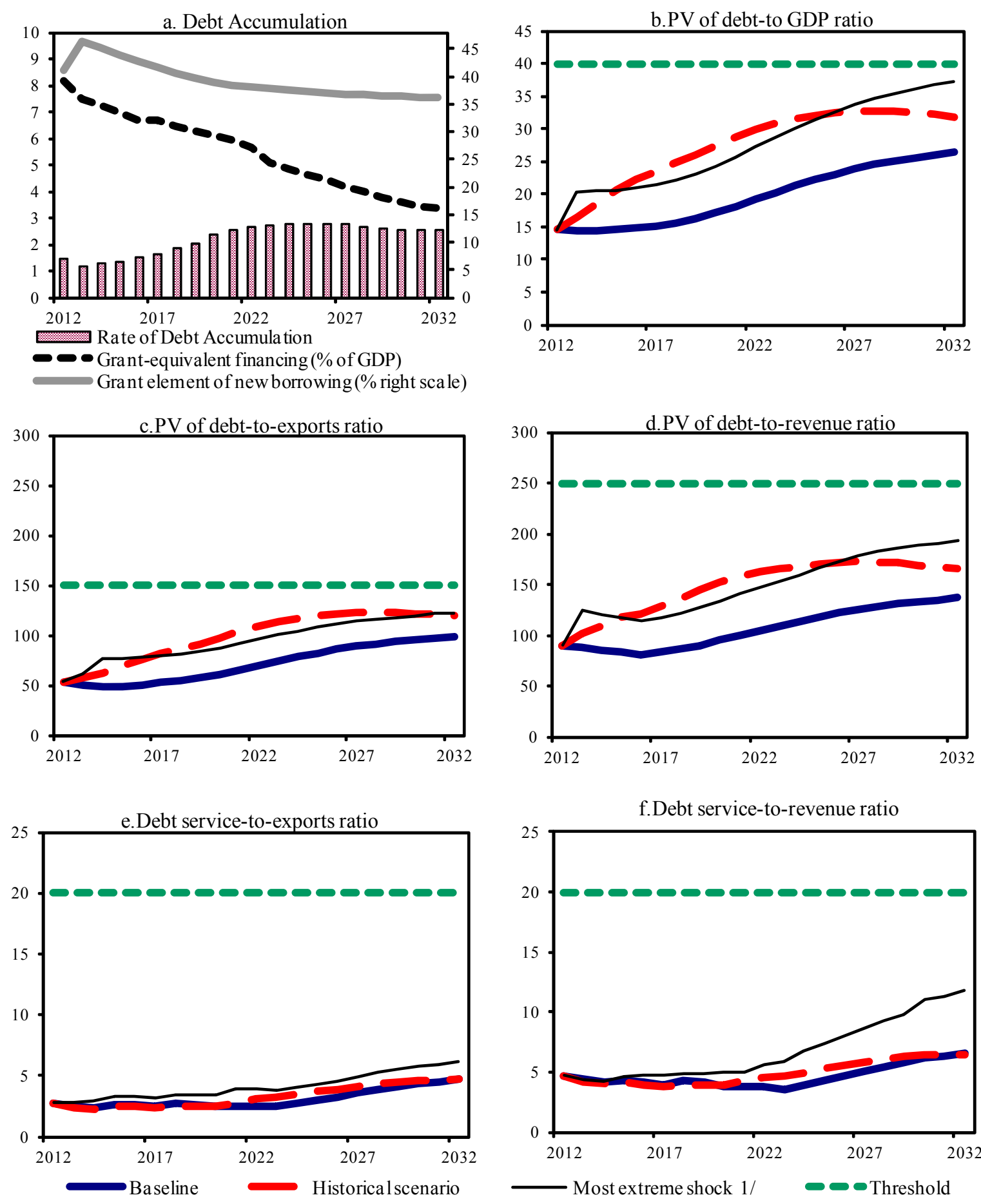

Sources: Country authorities; and staff estimates and projections.

$1 /$ The most extreme stress test is the test that yields the highest ratio in 2022. In figure b. it corresponds to a One-time depreciation shock; in c. to a Customized scenario (lower prices for 5 years and LT production decrease) shock; in d. to a Onetime depreciation shock; in e. to a Exports shock and in figure f. to a Terms shock 
Table 5a.Burkina Faso: Total Public Sector Debt Sustainability Framework, Baseline Scenario, 2009-2032

(In percent of GDP, unless otherwise indicated)

\begin{tabular}{|c|c|c|c|c|c|c|c|c|c|c|c|c|c|c|c|}
\hline & \multicolumn{3}{|c|}{ Actual } & \multirow[b]{2}{*}{ Average $^{5 /}$} & \multirow[b]{2}{*}{$\begin{array}{c}\text { Std. } \\
\text { Dev. }\end{array}$} & \multicolumn{4}{|l|}{ Estimate } & \multicolumn{3}{|c|}{ Projections } & & & \multirow[b]{2}{*}{$\begin{array}{l}2018-32 \\
\text { Average }\end{array}$} \\
\hline & 2009 & 2010 & 2011 & & & 2012 & 2013 & 2014 & 2015 & 2016 & 2017 & $\begin{array}{l}2012-17 \\
\text { Average }\end{array}$ & 2022 & 2032 & \\
\hline $\begin{array}{l}\text { Public sector debt } 1 / \\
\text { o/w foreign-currency denominated }\end{array}$ & $\begin{array}{l}26.1 \\
22.0\end{array}$ & $\begin{array}{l}27.1 \\
23.9\end{array}$ & $\begin{array}{l}29.3 \\
24.1\end{array}$ & & & $\begin{array}{l}28.3 \\
24.0\end{array}$ & $\begin{array}{l}27.2 \\
23.8\end{array}$ & $\begin{array}{l}26.0 \\
23.9\end{array}$ & $\begin{array}{l}25.3 \\
24.1\end{array}$ & $\begin{array}{l}24.9 \\
24.4\end{array}$ & $\begin{array}{l}25.1 \\
24.9\end{array}$ & & $\begin{array}{l}30.2 \\
30.2\end{array}$ & $\begin{array}{l}38.1 \\
38.1\end{array}$ & \\
\hline Change in public sector debt & 2.5 & 1.0 & 2.2 & & & -1.0 & -1.2 & -1.1 & -0.7 & -0.4 & 0.2 & & 1.3 & 0.4 & \\
\hline Identified debt-creating flows & 1.5 & 3.4 & 0.5 & & & 0.6 & 0.9 & 0.8 & 1.0 & 0.5 & 0.7 & & 1.4 & 0.2 & \\
\hline Primary deficit & 4.3 & 4.1 & 2.0 & 1.8 & 6.7 & 3.0 & 2.8 & 2.6 & 2.8 & 2.3 & 2.4 & 2.6 & 3.5 & 2.7 & 3.1 \\
\hline Revenue and grants & 19.6 & 20.1 & 21.8 & & & 23.2 & 22.7 & 23.2 & 23.3 & 23.8 & 23.7 & & 22.5 & 21.1 & \\
\hline of which: grants & 5.9 & 4.6 & 5.3 & & & 7.1 & 6.4 & 6.1 & 5.8 & 5.5 & 5.4 & & 4.1 & 1.9 & \\
\hline Primary (noninterest) expenditure & 23.9 & 24.2 & 23.8 & & & 26.2 & 25.6 & 25.8 & 26.1 & 26.1 & 26.1 & & 26.0 & 23.8 & \\
\hline Automatic debt dynamics & -2.1 & -0.5 & -1.5 & & & -2.4 & -1.9 & -1.8 & -1.8 & -1.7 & -1.7 & & -2.0 & -2.5 & \\
\hline Contribution from interest rate/growth differential & -0.8 & -2.1 & -2.0 & & & -2.4 & -2.0 & -1.9 & -1.9 & -1.8 & -1.8 & & -2.0 & -2.5 & \\
\hline of which: contribution from average real interest rate & -0.1 & -0.2 & -0.9 & & & -0.5 & -0.1 & -0.1 & -0.2 & -0.2 & -0.2 & & -0.3 & -0.4 & \\
\hline of which: contribution from real GDP growth & -0.7 & -1.9 & -1.1 & & & -1.9 & -1.9 & -1.8 & -1.7 & -1.6 & -1.6 & & -1.7 & -2.1 & \\
\hline Contribution from real exchange rate depreciation & -1.3 & 1.7 & 0.5 & & & 0.0 & 0.0 & 0.1 & 0.1 & 0.1 & 0.1 & & $\ldots$ & $\ldots$ & \\
\hline Other identified debt-creating flows & -0.8 & -0.2 & 0.0 & & & 0.0 & 0.0 & 0.0 & 0.0 & 0.0 & 0.0 & & 0.0 & 0.0 & \\
\hline Privatization receipts (negative) & -0.8 & -0.2 & 0.0 & & & 0.0 & 0.0 & 0.0 & 0.0 & 0.0 & 0.0 & & 0.0 & 0.0 & \\
\hline Recognition of implicit or contingent liabilities & 0.0 & 0.0 & 0.0 & & & 0.0 & 0.0 & 0.0 & 0.0 & 0.0 & 0.0 & & 0.0 & 0.0 & \\
\hline Debt relief (HIPC and other) & 0.0 & 0.0 & 0.0 & & & 0.0 & 0.0 & 0.0 & 0.0 & 0.0 & 0.0 & & 0.0 & 0.0 & \\
\hline Other (specify, e.g. bank recapitalization) & 0.0 & 0.0 & 0.0 & & & 0.0 & 0.0 & 0.0 & 0.0 & 0.0 & 0.0 & & 0.0 & 0.0 & \\
\hline Residual, including asset changes & 1.0 & -2.4 & 1.6 & & & -1.6 & -2.1 & -1.9 & -1.7 & -0.9 & -0.5 & & -0.1 & 0.1 & \\
\hline \multicolumn{16}{|l|}{ Other S ustainability Indicators } \\
\hline PV of public sector debt & $\ldots$ & $\ldots$ & 19.7 & & & 18.9 & 17.8 & 16.6 & 15.8 & 15.3 & 15.4 & & 19.3 & 26.4 & \\
\hline $\mathrm{o} / \mathrm{w}$ foreign-currency denominated & $\ldots$ & $\ldots$ & 14.6 & & & 14.5 & 14.4 & 14.5 & 14.6 & 14.8 & 15.2 & & 19.3 & 26.4 & \\
\hline $\mathrm{o} / \mathrm{w}$ external & $\ldots$ & $\ldots$ & 14.6 & & & 14.5 & 14.4 & 14.5 & 14.6 & 14.8 & 15.2 & & 19.3 & 26.4 & \\
\hline PV of contingent liabilities (not included in public sector debt) & $\ldots$ & $\ldots$ & $\ldots$ & & & $\ldots$ & $\ldots$ & $\ldots$ & $\ldots$ & $\ldots$ & $\ldots$ & & $\ldots$ & $\ldots$ & \\
\hline Gross financing need $2 /$ & 5.4 & 5.4 & 3.4 & & & 4.6 & 4.4 & 4.4 & 4.3 & 3.7 & 3.4 & & 4.2 & 4.0 & \\
\hline $\mathrm{PV}$ of public sector debt-to-revenue and grants ratio (in percent) & $\ldots$ & $\ldots$ & 90.5 & & & 81.5 & 78.1 & 71.6 & 67.8 & 64.2 & 64.9 & & 85.4 & 125.0 & \\
\hline PV of public sector debt-to-revenue ratio (in percent) & $\ldots$ & $\ldots$ & 119.4 & & & 117.2 & 108.8 & 97.3 & 90.4 & 83.5 & 84.2 & & 104.4 & 137.0 & \\
\hline o/w external 3/ & & & 88.2 & & & 90.2 & 88.4 & 84.8 & 83.3 & 80.9 & 83.3 & & 104.4 & 137.0 & \\
\hline Debt service-to-revenue and grants ratio (in percent) 4/ & 5.2 & 6.7 & 6.3 & & & 6.9 & 7.0 & 7.7 & 6.8 & 6.2 & 4.2 & & 3.1 & 6.0 & \\
\hline Debt service-to-revenue ratio (in percent) $4 /$ & 7.4 & 8.6 & 8.3 & & & 9.9 & 9.7 & 10.5 & 9.1 & 8.1 & 5.5 & & 3.7 & 6.6 & \\
\hline Primary deficit that stabilizes the debt-to-GDP ratio & 1.8 & 3.1 & -0.1 & & & 3.9 & 4.0 & 3.8 & 3.5 & 2.7 & 2.2 & & 2.2 & 2.4 & \\
\hline \multicolumn{16}{|l|}{ Key macroeconomic and fiscal assumptions } \\
\hline Real GDP growth (in percent) & 3.0 & 7.9 & 4.2 & 5.6 & 1.9 & 7.0 & 7.0 & 7.0 & 7.0 & 6.9 & 6.8 & 7.0 & 6.4 & 6.0 & 6.3 \\
\hline Average nominal interest rate on forex debt (in percent) & 1.1 & 1.1 & 1.2 & 0.9 & 0.3 & 0.9 & 0.9 & 0.9 & 0.9 & 0.9 & 0.9 & 0.9 & 0.9 & $0.9^{\prime \prime}$ & 0.9 \\
\hline Average real interest rate on domestic debt (in percent) & 5.9 & 2.8 & 1.7 & 3.0 & 3.4 & 2.3 & 3.6 & 3.6 & 3.4 & 3.2 & $2.3^{\prime \prime}$ & 3.0 & 1.0 & $1.0^{\circ}$ & 1.0 \\
\hline Real exchange rate depreciation (in percent, + indicates depreciation) & -6.7 & 8.3 & 2.3 & -3.3 & 10.2 & 0.1 & $\ldots$ & $\ldots$ & $\ldots$ & $\ldots$ & $\ldots$ & $\ldots$ & & & $\ldots$ \\
\hline Inflation rate (GDP deflator, in percent) & 2.3 & 2.8 & 5.6 & 3.0 & 2.8 & 3.5 & 2.0 & 2.0 & 2.0 & 2.0 & 2.0 & 2.3 & 2.0 & 2.0 & 2.0 \\
\hline Growth of real primary spending (deflated by GDP deflator, in percent) & 0.2 & 0.1 & 0.0 & 0.1 & 0.1 & 0.2 & 0.0 & 0.1 & 0.1 & 0.1 & 0.1 & 0.1 & 0.1 & 0.1 & 0.1 \\
\hline Grant element of new external borrowing (in percent) & $\ldots$ & $\ldots$ & $\ldots$ & ... & & 41.1 & 46.4 & 45.2 & 43.8 & 42.7 & 41.7 & 43.5 & 38.1 & 36.2 & \\
\hline
\end{tabular}

1/M edium term and long term gertiest astima

2/ Gross financing need is defined as the primary deficit plus debt service plus the stock of short-term debt at the end of the last period.

3/ Revenues excluding grants.

4/ Debt service is defined as the sum of interest and amortization of medium and long-term debt.

5/ Historical averages and standard deviations are generally derived over the past 10 years, subject to data availability. 
Table 5b.Burkina Faso: Sensitivity Analysis for Key Indicators of Public Debt 2012-2032

PV of Debt-to-GDP Ratio
Baseline
A. Alternative scenarios
A1. Real GDP growth and primary balance are at historical averages
A2. Primary balance is unchanged from 2012
A3. Permanently lower GDP growth 1/
B. Bound tests
B1. Real GDP growth is at historical average minus one standard deviations in 2013-2014
B2. Primary balance is at historical average minus one standard deviations in 2013-2014
B3. Combination of B1-B2 using one half standard deviation shocks
B4. One-time 30 percent real depreciation in 2013
B5. 10 percent of GDP increase in other debt-creating flows in 2013

Projections

\begin{tabular}{llllllll}
2012 & 2013 & 2014 & 2015 & 2016 & 2017 & 2022 & 2032 \\
\hline
\end{tabular}

PV of Debt-to-Revenue Ratio 2/

\section{Baseline}

19

19

19

19
$9 \quad 18$

17

16

15

15

\section{A. Alternative scenarios}

A1. Real GDP growth and primary balance are at historical averages A2. Primary balance is unchanged from 2012

A3. Permanently lower GDP growth 1/

$\begin{array}{lll}19 & 19 & 19 \\ 19 & 21 & 23 \\ 19 & 20 & 20 \\ 19 & 23 & 2 \\ 19 & 24 & 22\end{array}$

19
23
20
21
22

20

18

22

20

20

21

19
21
19
19
20

$\begin{array}{lll}19 & 26 & 37 \\ 21 & 24 & 29 \\ 20 & 25 & 35 \\ 18 & 19 & 23 \\ 20 & 23 & 28\end{array}$

\section{B. Bound tests}

B1. Real GDP growth is at historical average minus one standard deviations in 2013-2014

B2. Primary balance is at historical average minus one standard deviations in 2013-2014

B3. Combination of B1-B2 using one half standard deviation shocks

B4. One-time 30 percent real depreciation in 2013

B5. 10 percent of GDP increase in other debt-creating flows in 2013

Debt Service-to-Revenue Ratio 2/

\section{Baseline}

$\begin{array}{rrrrrrrr}81 & 76 & 68 & 62 & 58 & 58 & 65 & 85 \\ 81 & 79 & 73 & 70 & 68 & 70 & 88 & 123 \\ 81 & 78 & 73 & 69 & 67 & 68 & 98 & 170\end{array}$

\section{A. Alternative scenarios}

A1. Real GDP growth and primary balance are at historical averages A2. Primary balance is unchanged from 2012

A3. Permanently lower GDP growth $1 /$

$\begin{array}{rrrrrrrr}81 & 81 & 79 & 78 & 77 & 80 & 114 & 175 \\ 81 & 93 & 101 & 96 & 90 & 90 & 105 & 136 \\ 81 & 85 & 86 & 83 & 81 & 83 & 111 & 163 \\ 81 & 102 & 92 & 85 & 78 & 76 & 85 & 111 \\ 81 & 105 & 96 & 91 & 86 & 86 & 102 & 134\end{array}$

\section{B. Bound tests}

B1. Real GDP growth is at historical average minus one standard deviations in 2013-2014

B2. Primary balance is at historical average minus one standard deviations in 2013-2014

B3. Combination of B1-B2 using one half standard deviation shocks

B4. One-time 30 percent real depreciation in 2013

B5. 10 percent of GDP increase in other debt-creating flows in 2013

$\begin{array}{llllllll}7 & 7 & 8 & 7 & 6 & 4 & 3 & 5 \\ 7 & 7 & 8 & 7 & 6 & 4 & 3 & 6 \\ 7 & 7 & 8 & 7 & 6 & 4 & 3 & 7\end{array}$

Sources: Country authorities; and staff estimates and projections.

1/ Assumes that real GDP growth is at baseline minus one standard deviation divided by the square root of the length of the projection period.

2/ Revenues are defined inclusive of grants. 
Figure 3. Burkina Faso: Indicators of Public Debt Under Alternative Scenarios, 2012-2032 1/
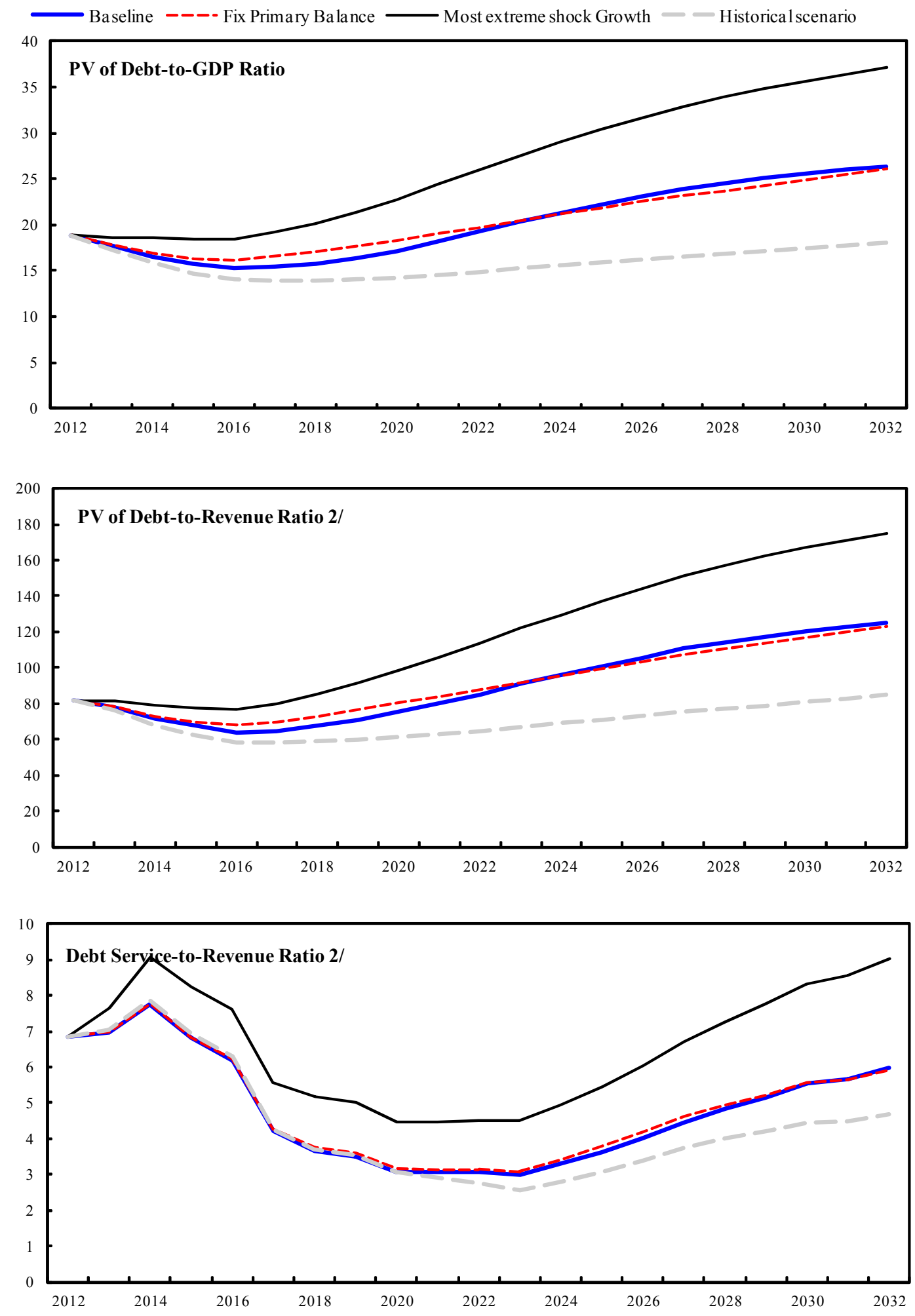

Sources: Country authorities; and staff estimates and projections.

1/ The most extreme stress test is the test that yields the highest ratio in 2022.

2/ Revenues are defined inclusive of grants. 
Press Release No. 12/214

International Monetary Fund

FOR IMMEDIATE RELEASE

Washington, D.C. 20431 USA

June 8, 2012

\section{IMF Executive Board Completes Fourth Review Under Extended Credit Facility Arrangement for Burkina Faso and Approves US\$46.1 Million Disbursement}

The Executive Board of the International Monetary Fund (IMF) today completed the fourth review of Burkina Faso's economic performance under the program supported by the Extended Credit Facility (ECF) arrangement. In completing the review, the Board also approved an augmentation of access equal to 60 percent of quota, an amount equivalent to SDR 36.12 million (about US\$54.6 million). The Board's approval of the augmentation would lead to a total access in an amount equivalent to SDR 82.274 million (about US\$124.3 million; or 136.67 percent of quota), under the ECF arrangement.

The completion of the review will enable the disbursement of an amount equivalent to SDR 30.53 million (about US\$46.1 million), bringing total disbursements under the arrangement to an amount equivalent to SDR 57.334 million (about US\$86.6 million).

The Executive Board approved the three-year ECF Arrangement for Burkina Faso on June 14, 2010 for an original amount of total access equivalent to SDR 46.154 million (about US\$69.7 million; or 76.67 percent of quota) (See Press Release No. 10/241).

Following the Executive Board's discussion on Burkina Faso, Mr. Min Zhu, Deputy Managing Director and Acting Chair, issued the following statement:

"The Burkinabé authorities are to be commended for their strong performance under the program in the face of continuing challenges. In 2011, despite a slowdown, growth remained above regional averages, and the fiscal deficit was reduced. The authorities put public finances on a more stable footing by improving revenue collection and reducing fuel subsidies. Measures were also taken to contain the public wage bill and other current spending, while improving overall public financial management, including spending execution.

"Burkina Faso is facing two shocks: inadequate food supplies and an influx of refugees. The authorities are implementing a comprehensive and well-targeted set of measures to meet 
domestic food security needs. However, the refugees are facing increasingly dire living conditions, requiring redoubled efforts from the authorities and the international community.

"The immediate increase in financing under the Fund-supported program will help meet import needs arising from the crises. This is also intended to safeguard spending for Burkina Faso's homegrown development program, the Stratégie pour une Croissance Accélérée et pour le Développement Durable (SCADD). The SCADD contains a number of welcome measures to improve resilience and make growth more sustainable and inclusive, notably through diversifying agricultural production, expanding irrigation systems, improving food distribution systems, and introducing a social protection framework for the most vulnerable households. The authorities should accelerate reforms to boost private sector led growth, in particular strengthening the judicial framework.

"New analysis by the IMF and World Bank resulted in lowering Burkina Faso's risk of debt distress rating from "high" to "moderate." This opens the door to new financing opportunities, other than grants, but implies that care is required when choosing projects and evaluating financing in order to maximize growth returns and to keep public debt sustainable," Mr. Zhu added. 


\section{Statement by Mr. Assimaidou, Executive Director for Burkina Faso June 8, 2012}

My Burkina Faso's authorities thank staff, Management, and the Executive Board, for their continued support in their efforts to implement the Extended Credit Facility (ECF) program. They are very appreciative of the constructive policy dialogue held in Ouagadougou with Staff in the context of the last review mission.

Burkina Faso's authorities have continued to demonstrate a strong commitment to prudent policies and sound reforms, amidst a challenging environment due to the instability in the region, and the weakening of the global economy. In this context, all program performance criteria were met. Moreover, all indicative targets were implemented, except for the one related to social expenditures, which was missed owing to delays in the mobilization of resources. Most of the structural benchmarks were also achieved.

Although growth has recovered, mounted social demands, geo-political crises in the subregion, the food shortages brought by low rainfalls, as well as the increased international oil prices, are generating additional pressures on the limited resources available. The conflict in Mali in particular has so far, led to an inflow of more than 57,000 refugees in Burkina Faso. In addition to the current challenges, the authorities continue to face the daunting task of making inroads towards the Millennium Development Goals.

In order to address the balance of payments needs arising from the response to the crisis, and preserve macroeconomic stability, while pursuing their growth and sustainable development strategy (SCADD), the authorities are requesting an augmentation of access under the existing ECF arrangement, and the completion of the fourth review.

\section{Recent Economic Developments}

Real GDP growth decelerated markedly in 2011, settling at 4.2 percent, well below the expected 7.9 percent, due to unfavorable weather conditions and the social unrest in the first half of the year. The policy environment in 2012 has also been challenging due to political instability in the sub-region, and the poor rainy season which caused a food crisis in West Africa.

The authorities responded to the different challenges by taking initiatives to appease social tensions at home, and by conducting mediations aimed at reducing geo-political tensions in the sub-region, in close partnership with the United Nations, and regional organizations.

The inflow of refugees overwhelmed the capacities of NGOs and other agencies which are endeavoring to assist them, and the government took the lead to supplement their efforts until 
further resources become available from the UN and other donors. The authorities also took measures to address food insecurity stemming from this years' drought. All these measures have severely tested the capacity of Burkina Faso. Nevertheless, program implementation has been maintained.

On fiscal policy, despite the difficult social context, the authorities managed to meet the fiscal deficit objective. They exceeded their revenue target notably through reforms aimed at improving tax and customs administration. In order to reduce fraud and increase compliance, scanners were deployed at customs offices, the SYLVIE system was launched, and efforts were made which helped reduce the large taxpayer non-filer rate to 3 percent, well below the initial goal of 5 percent.

Going forward, recent adoption of a new petroleum pricing mechanism, and increased retail oil prices, will be instrumental in sustaining strong revenue performance and protecting public finances, notably by ensuring the pass through of international oil prices to domestic prices.

On spending, the government stepped up efforts to control spending and improve its efficiency, including through the expansion of auditing activities of line ministries, and improved payroll management.

The authorities continued to contract external financing only on concessional terms, with a view to preserving debt sustainability. They also pursued efforts to improve their debt management capabilities by implementing the recommendations made by the 2011 World Bank's DeMPA mission. Notably, they conducted training sessions on risk management, and on SYGADE to improve cash flow management and forecasting.

As regards financial sector policies, the authorities sought to improve access to financial services by adopting a new microfinance development strategy, and an implementation plan.

On structural reforms, the authorities' actively implemented their strategy for accelerated growth and sustainable development (SCADD). They inaugurated the Bagré growth pole, a pilot project designed to use synergies and economies of scale in an integrated set of rural development projects. They also continued to improve governance and transparency in the natural resources sector, in line with EITI's recommendations. Regarding cotton, a strategy was adopted with the goal of reducing the public sector's stake in the sector.

\section{Policies for the Remainder of 2012}

The authorities will seek to mitigate the impact of the crises, and to strengthen the economy's resilience to future shocks, while pursing the implementation of their accelerated growth and development strategy, SCADD. 


\section{Fiscal Policy}

Fiscal policy will aim at creating the fiscal space needed to finance growth enhancing priority investments, and to meet crisis related increases in social spending, while preserving medium term fiscal sustainability.

The government plans to carry forward the remaining phases of the food crisis response, and to allocate additional resources for refugees assistance operations. Beyond spending related to crises response, the authorities will re-allocate resources towards public investment projects identified as critical by SCADD, including rural development infrastructures such as dams and irrigation systems. They will also aim at controlling spending and enhancing further its efficiency, including through improvements of budget control processes and in the procurement systems.

On the revenue side, the authorities will pursue efforts to improve revenue collections. In this regard, they plan to consolidate reforms underway, and to continue the modernization of the customs and revenue administration offices, and to deploy additional modules on the customs management software. They also plan to step up their efforts to reduce fraud and further ensure tax compliance. To that effect, tax collection units will be added, and the coverage of tax audits will be expanded. Efforts will also be made to install customs units at mining sites, and improve their inspection mechanism.

\section{Debt policies}

The authorities remain committed to the preservation of debt sustainability. They welcome the reclassification of Burkina in the new DSA as at "moderate risk" of debt distress, which results from years of adherence to prudent borrowing policies and the support of their development partners in the form of grants and concessional lending. Looking forward they are committed to further improve this record through sound debt policies, and the strengthening of their debt management capacity.

They plan to continue to seek financing for priority projects on concessional terms or in the form of grants. However, given the increasingly limited availability of concessional resources, reflecting the impact of the global financial crisis on traditional donors' finances, the authorities intend to tap non-concessional resources for some profitable investment projects.

\section{Financial Sector Policies}

As regards the financial sector, the authorities will intensify their efforts to increase access to the financial services, including for small and medium-sized enterprises. They will also 
closely monitor the transition of the banking system to the new regulatory framework requiring added capital ratios.

\section{Structural reforms}

The authorities plan to further their ambitious structural reforms agenda under the framework provided by the homegrown growth and poverty reduction strategy, SCADD. This growth and poverty reduction strategy provides for sustained investments in human capital, particularly in the health and education sectors. Along with building a skilled labor force, the authorities plan to launch additional regional growth clusters building on lessons learned from the pilot Bagré growth pole.

Each growth pole will harness the country's competitive advantage to build a diversified and striving economy. Investments in rural development, notably in water resources management, will help secure the food supply and reduce the economy's vulnerability to weather-related shocks. In addition to boosting productive sectors, the authorities plan to remove growth bottlenecks, by investing in transportation infrastructures and in improving the availability and affordability of energy supply.

They will also seek to promote private sector development, including through measures aimed at facilitating the creation and financing of small businesses, and at improving the business climate. On the latter, they will seek to reduce production factors costs, notably of energy, and to improve the legal framework for doing business.

Given the rapid growth of the mining sector's share in the economy, the authorities plan to implement reforms with the technical assistance of the World Bank and the IMF in order to make the best use of the natural resources in promoting a sustainable development. They intend to review the legal framework to improve transparency in the sector and harmonize the legislation with regional mining standards, while improving the management of mining permits. They also hope to realize the fiscal revenue potential of the mining sector and to leverage its contribution to their efforts to diversify the economy.

In the cotton sector, the authorities will seek the assistance of an investment bank to help implement their recently adopted strategy to reduce the government's stake in the sector. Higher international cotton prices and the sector's expansion could help improve SOFITEX's balance sheets.

\section{Conclusion}

In spite of a difficult policy making environment, marked by the food crisis, and geo-political tensions and a subsequent inflow of refugees, the authorities have steadfastly implemented their program of economic and financial reforms, with Fund's support. They remain 
committed to sound policies going forward, to accelerate growth and reduce poverty while increasing the economy's resilience to future shocks. To assist their reform efforts, they are requesting the support of the Board for the completion of the fourth review under the ECF arrangement, the modification of performance criteria, and an augmentation of access to help cope with balance of payments needs stemming from the crises.

Given the strong commitment of the authorities to the ongoing program, and the determination of the authorities to achieve their growth and poverty reduction objectives, I would appreciate the support of Directors to the requests of my Burkinabé authorities. 